\title{
LEARNING TO EXPECT THE PREDICTABLE: THE ROLE OF EXPECTATION IN THE COGNITIVE CONTROL OF ATTENTION
}

BY

\section{ANDRÉ BOTES}

\author{
A thesis \\ submitted to the Victoria University of Wellington \\ in fulfilment of the requirements for the degree of \\ Master of Science in
} Cognitive and Behavioural Neuroscience

Victoria University of Wellington 2020 



\begin{abstract}
Some of the visual world is relevant to our goals and needs. Much more is not. A problem we face frequently in day-to-day living is that we are distracted by what is not relevant to our goals at the cost of attention towards what is. Emotional stimuli in particular have been shown to be very effective distractors, out-competing task-relevant stimuli for our attentional resources (Carretié, 2014; Pessoa, 2005; Pourtois et al., 2013).

How often emotional distractors occur can alter our ability to ignore them and remain task focussed (Grimshaw et al., 2018; Schmidts et al., 2020). The Dual Mechanisms of Control framework (Braver et al., 2007; Braver, 2012) suggests that, because we can expect upcoming distractors when they occur frequently, we can effectively avoid distraction through proactive control; the use of effortful preparatory cognitive control strategies.

That said, when distractors are frequent, we also become more experienced with them, and resolving the attentional conflict they create. The present investigation spanned two experiments assessing whether expectation of upcoming distractors would elicit proactive control while holding the experience of previous distractors constant. In Experiment 1 participants performed a simple perceptual task at fixation while neutral or negative taskirrelevant images appeared peripherally on $25 \%$ of trials, either predictably in sequence (every fourth trial) or randomly. Expectation of distraction did not improve participants' ability to avoid emotional distraction. A paradoxical expectation effect was also found wherein distraction was increased rather than decreased when distractors occurred predictably.

In Experiment 2 distractors appeared either predictably (every fourth trial), on a random $25 \%$ of trials, or on a random $75 \%$ of trials. However, neutral and emotional images were now presented at fixation with the perceptual task presented above and below. Greater distractor frequency led to lower distraction and expectation of upcoming distractors again did not improve control, although a paradoxical increase in distraction was not replicated.

Findings indicate that expectation of upcoming distractors alone is not sufficient to drive individuals to implement proactive control. Rather, distractor frequency is suggested to drive proactive control through implicit changes in top-down control settings based on experience. While the processes behind experience-driven proactive control are unclear, conflict adaptation and selection history are discussed as possible mechanisms of experiencedriven proactive control. Critically, present findings also indicate that emotional stimuli may present a unique challenge to our ability to control our attention.
\end{abstract}




\section{Acknowledgements}

Thank you to Gina Grimshaw for taking the time and effort to teach me how to do this all properly. For your contributions to my understanding and development in science, you have my sincerest gratitude. I would also like to thank Paul Corballis for being well worth listening to and for giving me my first real project. I could not have asked for a better start.

Thank you to my family who provided solid support over the last few years. The process of my writing this thesis has not been a quick or efficient one and I would not have managed to get this far without their help and steadfastness.

Thank you to Ruby, without whom this would not have been written.

Thank you to the members of my CBNS cohort and the VUW CANlab for their time, patience, guidance, and willingness to help at all stages of this thesis.

Lastly, thank you to John, Callum, Ben, Ben, Ben, Sam, Rhiannon, Jazz, Hunter, Chiaki, and Mac for your fantastic work in either reading this whole thesis, the one before this one or simply for being a well appreciated distraction. It seems like you don't appreciate the company you keep until they prove themselves useful. 


\section{Table of Contents}

Abstract .

Learning to Expect the Predictable: The Role of Expectation in the Cognitive Control of

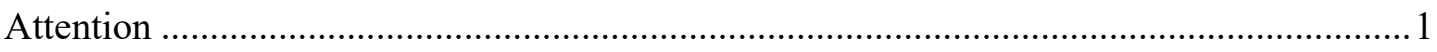

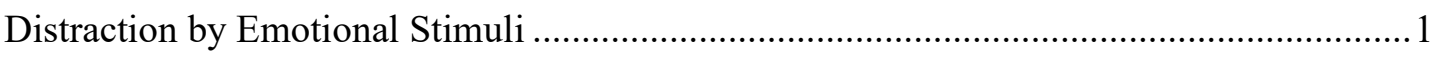

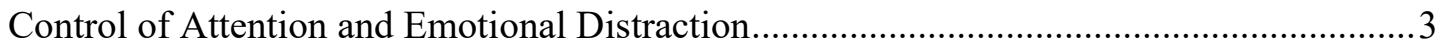

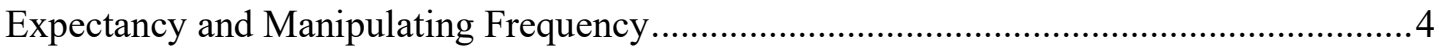

The Divide Between Experience and Expectation........................................................ 5

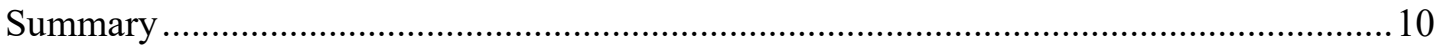

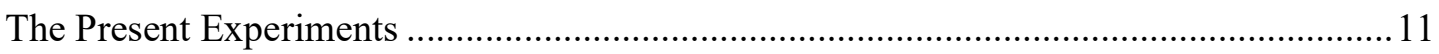

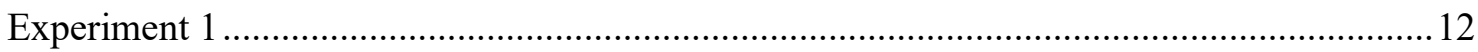

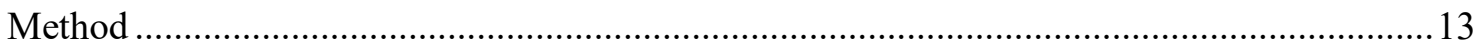

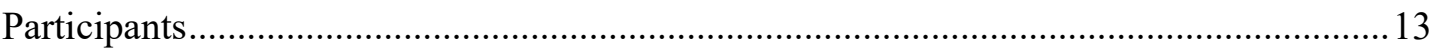

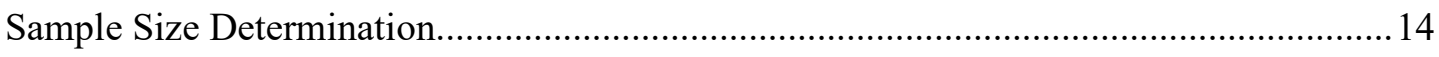

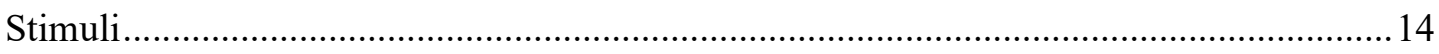

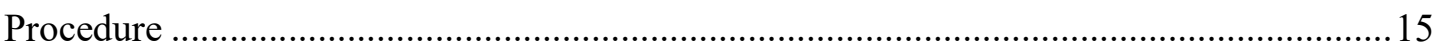

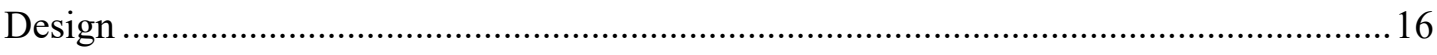

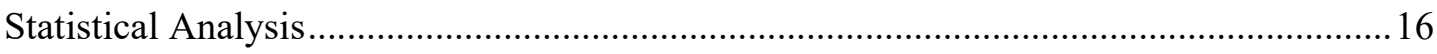

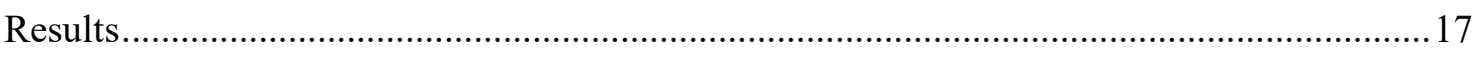

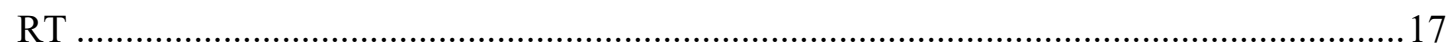

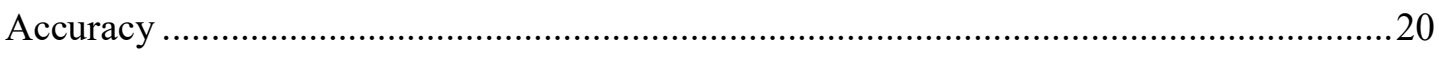

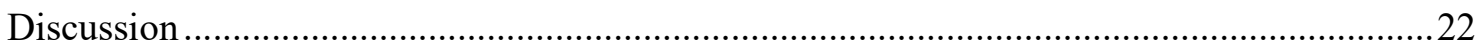

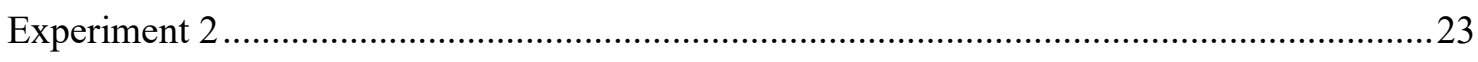

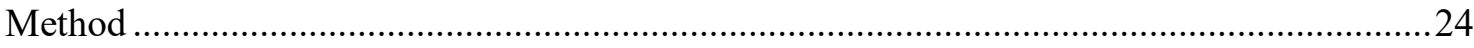

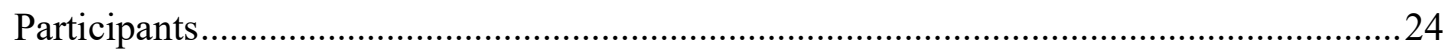

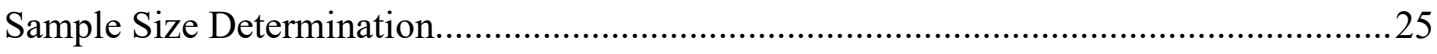

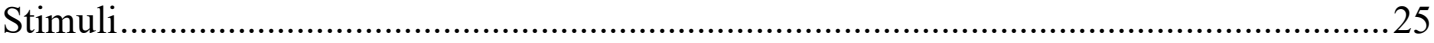




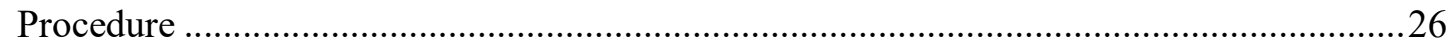

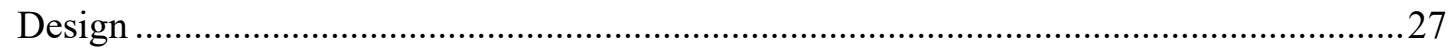

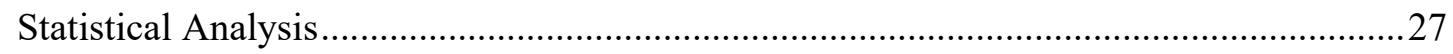

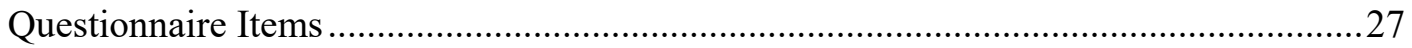

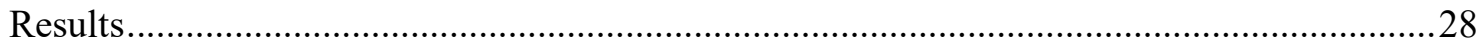

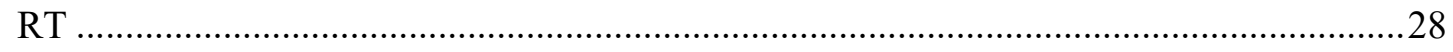

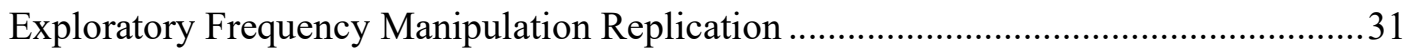

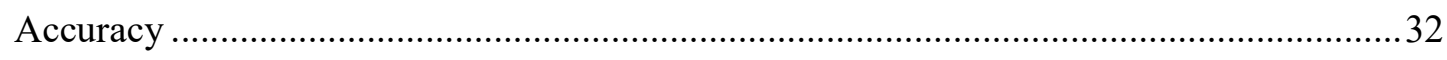

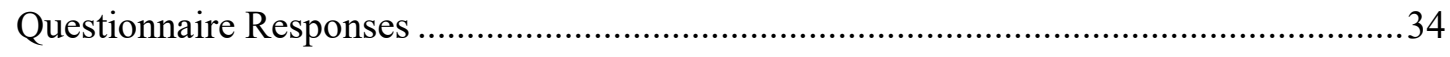

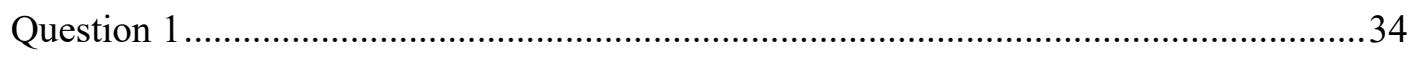

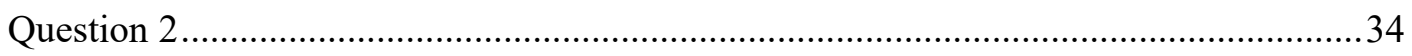

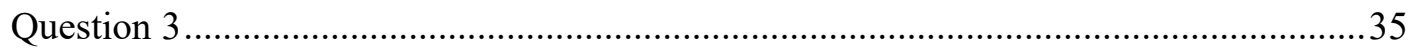

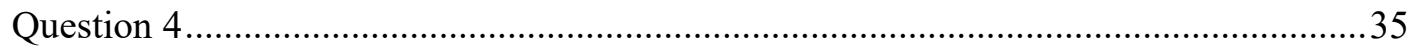

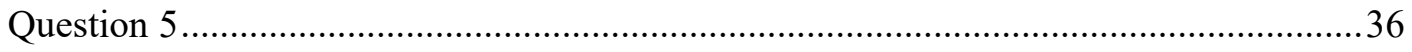

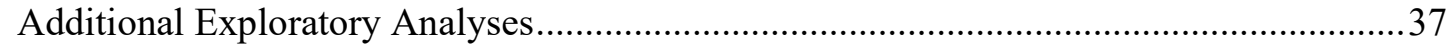

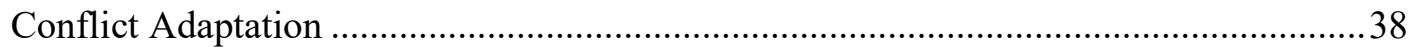

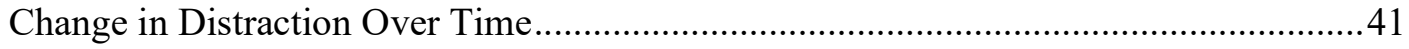

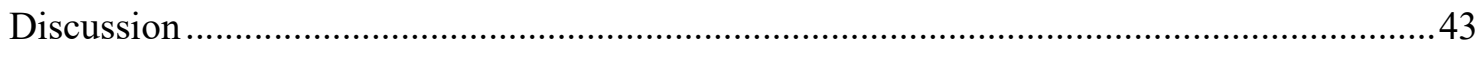

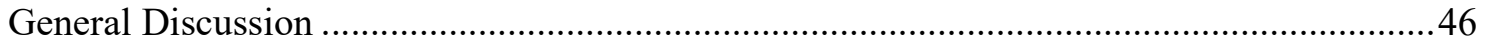

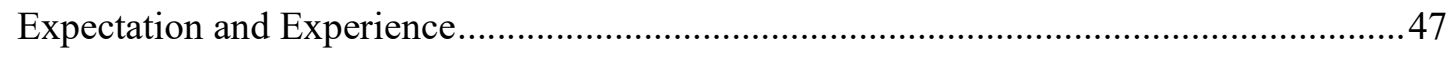

Evidence Against a Solely Experience-Based Account of Cognitive Control ....................49

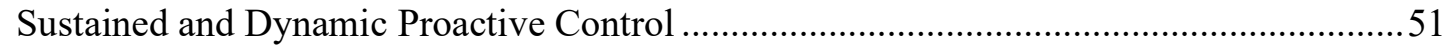

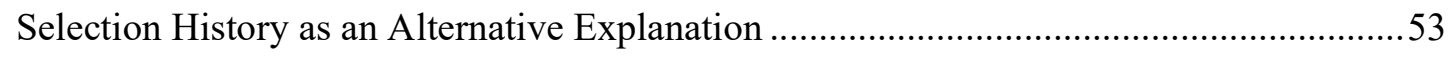

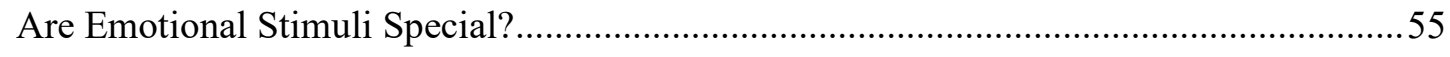

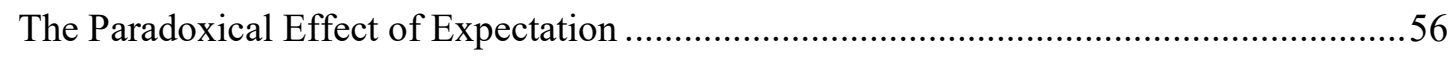

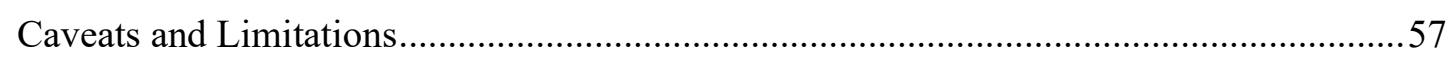

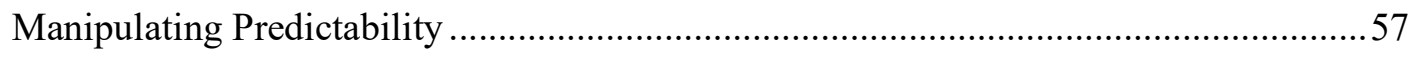

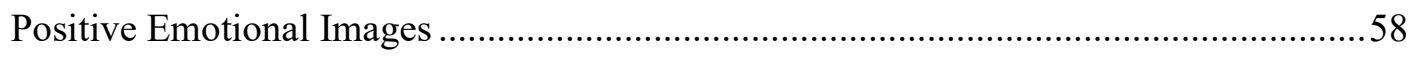

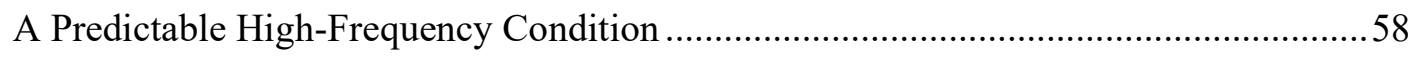

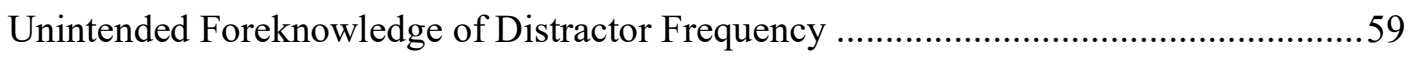

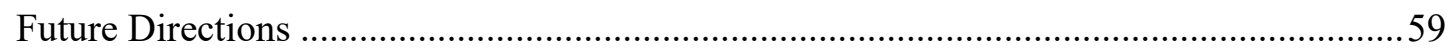




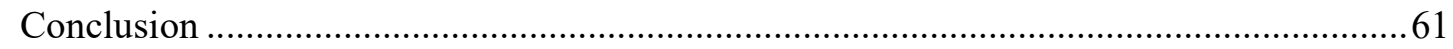

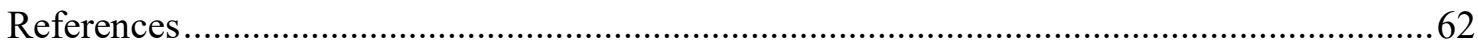

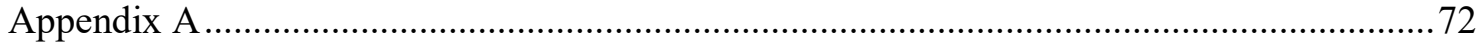

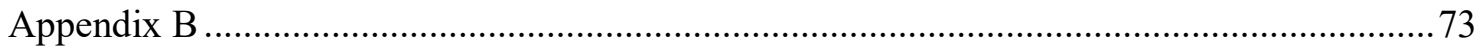

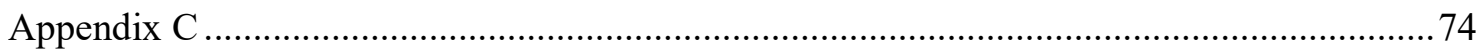

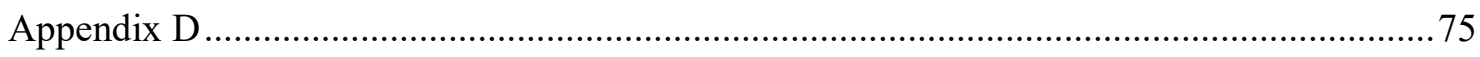

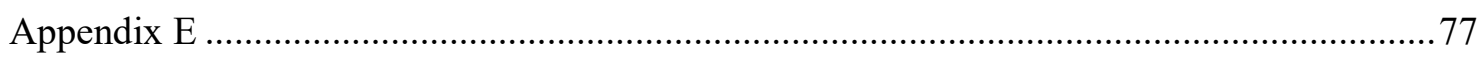




\section{List of Figures}

Figure 1. A) Trial structure of Experiment 1, showing a neutral distractor-present trial B) Examples of a distractor-absent trial (left) and a neutral distractor-present trial (right)

Figure 2. Experiment 1 individual RTs, by Predictability, Valence and Distractor presence .19

Figure 3. Experiment 1 individual Distraction indices by Valence and Predictability.

Figure 4. Experiment 1 individual Accuracy scores by Predictability, Valence and Distractor presence.

Figure 5. A) Trial structure of Experiment 2 showing a neutral intact-distractor trial B) Examples of a scrambled-distractor trial (left) and a neutral intact-distractor trial (right) 26

Figure 6. Experiment 2 individual RTs, by Predictability condition, Valence and Distractor type

Figure 7. Experiment 2 individual Distraction indices, by Predictability condition, Valence and Distractor type . 31

Figure 8. Experiment 2 individual Accuracy scores by Predictability condition, Valence and Distractor type . 34

Figure 9. A) Individual RTs by Previous trial type, Valence and Distractor type for trials in the unpredictable high-frequency condition of Experiment 2 B) Individual RTs by Previous trial type, Valence and Distractor type for trials in the unpredictable lowfrequency condition in Experiment 2

Figure 10. Experiment 2 scrambled-distractor trial RTs in the predictable low-frequency condition by Valence and Number of trials since an intact-distractor

Figure 11. Experiment 2 Distraction indices by Predictability condition, Valence and Block42

Figure 12. Experiment 2 RTs for the first block of the experiment divided into the first, middle and final 16 trials, by Predictability condition and Distractor type .43 


\section{List of Tables}

Table 1. Mean (SD) RTs (in ms), Distraction indices and paired samples t-test results, including confidence intervals and effect sizes (Cohen's $d_{z}$ ), by Valence, Predictability and Distractor presence in Experiment 1

Table 2. Mean (SD) accuracy scores (in \% correct trials) and paired samples t-test results, including confidence intervals and effect sizes (Cohen's $\mathrm{d}_{\mathrm{z}}$ ), by Valence, Predictability and Distractor presence in Experiment 1 21

Table 3. Mean (SD) RTs (in ms), Distraction indices and paired samples t-test results, including confidence intervals and effect sizes (Cohen's $\mathrm{d}_{\mathrm{z}}$ ), by Valence, Predictability

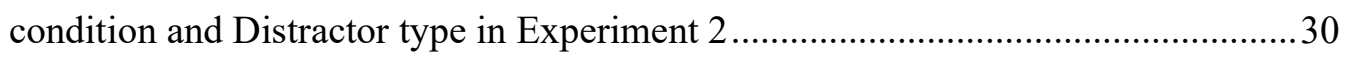

Table 4. Mean (SD) accuracy scores (in \% correct trials) and paired samples t-test results, including confidence intervals and effect sizes (Cohen's $\mathrm{d}_{\mathrm{z}}$ ), by Valence, Predictability Condition and Distractor type in Experiment 2 .................................33

Table 5. Tallies and percentages (\%) of responses to post-experiment questionnaire items

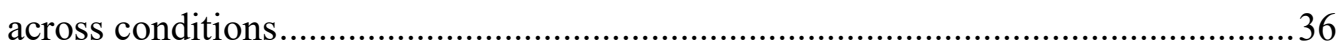

Table 6. Tallied responses to question 5 of the post-experiment questionnaires, separated by response similarity and Predictability condition. 


\section{Learning to Expect the Predictable: The Role of Expectation in the Cognitive Control of Attention}

To effectively navigate day-to-day living, we must be able to apply attention selectively, attending to what is relevant to our goals at the detriment of what is not. When our limited attentional resources are instead captured by that which is irrelevant, i.e. if we are distracted, our goals are harder to achieve. Emotional stimuli pose substantial risk of distraction because they are salient, acting as signals of threat and reward, and often irrelevant to our immediate goals (Carretié, 2014; Ohman et al., 2001; Schupp et al., 2007). However, when emotional stimuli outcompete contextually more relevant stimuli, how does our attention stay focussed on what it is supposed to be?

One factor which can affect our ability to avoid distraction is how often irrelevant emotional stimuli appear. When emotional stimuli occur frequently, we can effectively ignore them. What is unclear is why the frequency of these distractors modifies how well we control our attention. It is assumed that, when emotional distractors occur frequently, we are more able to expect and therefore prepare for them in advance. However, there is a problem with this assumption; when distractors are more frequent, we also gain more experience with them and the conflict they induce.

The divide between the influence of expectation and experience has implications as to how we understand the influence of emotion on selective attention and cognitive control. Furthermore, understanding how we control our attention in the face of potent distractors may provide insight into the psychopathology of those who experience greater susceptibility to emotional distraction (e.g. individuals high in psychopathy or individuals with depression; Kalanthroff et al., 2016; Mitchell et al., 2006; Wang et al., 2008).

In this thesis, I present two experiments which assess whether expectation of upcoming emotional distractors determines the type of control mechanisms used to ignore them and remain focussed on immediate goals. In Experiment 1 I compare distraction by emotional and neutral images when distractors are infrequent but unpredictable as opposed to infrequent but occurring in a predictable sequence. Experiment 2 is a replication of the first experiment which further compares performance when distractors are rare and predictable to a high-expectation, high-experience condition where distractors are frequent and unpredictable.

\section{Distraction by Emotional Stimuli}

Visual stimuli compete for a limited pool of attentional resources (Desimone \& Duncan, 1995). Attention and further processing are given to stimuli which can win out in this competition, at the cost of attention to others. Competition for attentional resources can be 
biased based on top-down goal-directed factors, bottom-up stimulus-derived features (such as movement or onset; Connor et al., 2004) or the history of previous attentional selection (Awh et al., 2012; Theeuwes, 2019). Thus, how we allocate our limited attentional resources depends on aspects of the stimuli, our previous attentional allocation, and our own goals, suggesting an intricate combination of environmental and self-generated biases.

Emotional stimuli (i.e. stimuli associated with emotional content, people, objects or events), often outcompete other stimuli for attentional resources (Brosch et al., 2010; Carretié, 2014; Carretié et al., 2004; Pool et al., 2016; Pourtois et al., 2013). But what drives this bias? Pourtois and colleagues (Pourtois et al., 2013), suggest that emotional stimuli are able to bias attentional competition, beyond the influences of top-down guidance and bottom-up features, due to their motivational value. As an example, negatively valenced or fear relevant emotional stimuli, such as gore or dangerous animals, can be indicative of bodily harm to the viewer, while positively valenced emotional stimuli, such as erotica, are linked to possible reward (Brosch et al., 2010; LeDoux, 2012; Ohman et al., 2001). Clearly, there is benefit to survival in quickly detecting the snake in the grass (Ohman et al., 2001) or seeking a reproductive partner (Most et al., 2007; Pessoa, 2009). Not only are we motivated to attend to emotional stimuli, it is evolutionarily adaptive to do so.

The inherent salience of emotional stimuli can however become problematic. We interact with many emotional stimuli in our day-to-day living and often these emotional stimuli are irrelevant to our needs or goals. Hence, we are often susceptible to emotional distraction, specifically defined here as the capture of attentional resources by emotional stimuli when they are irrelevant to current goals. A method that has been used to study emotional distraction is the emotional irrelevant-distractor paradigm (Grimshaw et al., 2018; Gupta et al., 2016; Walsh et al., 2018, 2019). In this adaptation of the irrelevant-distractor paradigm (Forster \& Lavie, 2008), participants are presented with a simple perceptual task (e.g. identify a letter within an array), accompanied on some proportion of trials by emotional or non-emotional distractor images. Key here is the level to which emotional and neutral stimuli differentially disrupt task performance. Typically, participants are slower in responding to the letter array when emotional relative to neutral images are used as the task-irrelevant distractor, indicating greater distraction by emotional than neutral stimuli.

Task performance can be seriously undermined when attentional resources should be directed toward stimuli which are task relevant but instead are captured by emotional and irrelevant distractors. The very value of emotional stimuli as environmental signals therefore 
also allows them to be very effective distractions. The important questions are then whether, and how, we can control attention and avoid emotional distraction.

\section{Control of Attention and Emotional Distraction}

Broadly, cognitive control ${ }^{1}$ refers to mechanisms which are implemented to generate and maintain task goals (Gratton et al., 2018), typically, in response to the presence of conflict in the environment (Braver, 2012; Gratton et al., 2018). Conflict arises when concurrent processes seek access to the same resources, as is the case when task-relevant and taskirrelevant visual stimuli compete for the same pool of attentional resources (Botvinick et al., 2001; Pessoa, 2009). In practice this means that adequate cognitive control can resolve the conflict between goal-relevant and goal-irrelevant stimuli (e.g. emotional distractors) in a way that favours goal attainment, regardless of the relative salience of the irrelevant stimulus.

The dual mechanisms of control (DMC) framework (Braver, 2012; Braver et al., 2007), suggests that the implementation of cognitive control is based on the relative use of two different types of control mechanisms: proactive and reactive. Proactive control involves the maintenance of goal relevant information, allowing individuals to bias attention or action optimally and prior to the occurrence of conflict. On the other hand, reactive control is corrective, used only after the occurrence of conflict to bring behaviour or attention back in line with goals.

A strength of the DMC framework is in its explanation of the dynamic nature of cognitive control; our ability to apply the appropriate levels of cognitive control given the changing demands of the environment. The relative use of either reactive or proactive mechanisms is thought to depend on a cost-benefit analysis of their use. Proactive control is more effective than reactive control but also more costly (Aron, 2011). Consequently, proactive control is only used when the benefit of proactive mechanisms offsets the cost of their implementation. Reactive control is then relied on as a "default state", leading to less costly but also less effective control that can be implemented responsively. In this way, control mechanisms can be appropriately "metered out" given the contextual need for their use.

The qualitatively distinct nature of proactive and reactive control has been shown by neuroimaging studies. The pre-frontal cortex (PFC) is thought to be critical to the implementation of top-down/executive control functions, which control cognition and behaviour in light of overall goals and needs (Gratton et al., 2018; Katsuki \& Constantinidis,

\footnotetext{
${ }^{1}$ Care should be taken to consider cognitive control in terms of the language used in the field (see Gratton et al., 2018, for discussion) but the terms attentional control, cognitive control and executive function are often used interchangeably. The term cognitive control will primarily be used from here on.
} 
2014). Reactive control is associated with post-conflict PFC activation, suggesting corrective action after conflict detection (Braver et al., 2009; Braver, 2012). Proactive control is often associated with sustained increases in PFC activation, reflecting the active maintenance of task goals (Braver et al., 2009). Hence effortful proactive control reflects sustained activity allowing individuals to be prepared to mitigate conflict or cognitive demand prior to its occurrence. However, evidence has also been shown for anticipatory/pre-conflict PFC activation, reflecting more dynamic proactive control, implemented prior to conflict occurrence (De Pisapia \& Braver, 2006).

\section{Expectancy and Manipulating Frequency}

Shifting the cost/benefit assessment in favour of a more proactive style of cognitive control can improve one's ability to control attention. As an example, Walsh et al. (2019) found less distraction, in terms of both accuracy and reaction time (RT) by both emotional and neutral task-irrelevant distractors when participants were rewarded for good task performance since the benefits of more costly control were increased. Previous studies find a similar shift to proactive control when conflict occurs frequently. It is assumed that when conflict is frequent, participants build an expectation of upcoming conflict (i.e. the explicit and advance knowledge of future likelihood of conflict) and can therefore prepare resources in advance (Braver, 2012; Bugg et al., 2015). The cost of preparation is then offset by the benefits of improved control (i.e. proactive control becomes cost-effective). Conversely, when conflict occurs rarely, there is lower expectation of upcoming conflict and the cost of proactive control is likely to be wasted.

Frequency effects have been previously shown in flanker, Simon, task switching and Stroop tasks (Bugg \& Crump, 2012; Duthoo et al., 2012; Gratton et al., 1992; Hutchison et al., 2016); examples of traditional response-conflict paradigms. The Stroop task (Stroop, 1935), is typically thought to be the gold standard of methods for assessing cognitive control. In the Stroop task, names of colours are presented to participants in a print colour that is congruent or incongruent with the word meaning. Participants must then report the colour in which the word is printed. The typical Stroop effect is seen when participants take longer to report the colour when the word and colour are incongruent as opposed to congruent. Frequency effects, commonly referred to as proportion congruency effects in Stroop studies (Jiang et al., 2014; Logan \& Zbrodoff, 1979), are achieved by altering the proportion of congruent and incongruent trials. The magnitude of the Stroop effect is typically seen to diminish when the proportion of incongruent trials is high (i.e. conflict is frequent) relative to when the proportion of incongruent trials is lower. 
Frequency effects have also been shown in the resolution of perceptual conflicts (i.e. the resolution of conflict between visual stimuli for attentional resources). In Geyer et al. (2008), participants were shown item arrays wherein a single shape (a green circle) contained a task relevant target. A salient distractor (a red square) was present concurrently on either $20 \%, 50 \%$ or $80 \%$ of trials. The difference in RT between the distractor-present and distractorabsent trials was significant in the $20 \%$ and $50 \%$ conditions but not in the $80 \%$ condition. Distraction was therefore substantially reduced when distractor frequency was high relative to when it was low. Similar results are reported using the same paradigm by Müller et al. (2009). Here initial exposure to a $100 \%$ distractor frequency block reduced distraction on all subsequent blocks of trials with sequentially decreasing distractor frequency, suggesting a sustained heightening of cognitive control. Distraction by neutral task-irrelevant stimuli can therefore be avoided when they occur frequently.

But what if we were inherently biased to attend to distractors? Does increased distractor frequency also improve control over the attentional conflict brought on by emotional distractors? An example, again, is the emotional irrelevant-distractor task used by Grimshaw et al. (2018), who found decreased distraction by positive emotional images (scenes of erotica), negative emotional images (scenes of mutilation and gore) and neutral images when distractors are presented frequently. Distraction by negative and positive emotional stimuli was greater than distraction by neutral stimuli when distractors were rare ( $25 \%$ of trials). In contrast, when distractors occurred frequently (75\%), distraction by images of all valences was low. Hence when distractors occurred frequently, so that participants had reasonable expectation of distraction on any given trial, emotional stimuli were ignored just as well as neutral stimuli.

Schmidts et al. (2020) observed similar findings in an irrelevant-distractor design wherein participants were asked to indicate if a letter, presented alongside an irrelevant distractor, preceded or followed the letter $\mathrm{M}$ in the alphabet. Trials were also preceded by either an informative or uninformative valence cue. Participants were shown trial blocks containing predominantly negative (emotional distractors are frequent) or predominantly neutral distractors (emotional distractors are rare). Considering only the uninformative cue trials, when negative distractors were rare, RT to negative distractors was substantially greater than to neutral distractors. However, when negative distractors occurred frequently, distraction by both negative and neutral trials was reduced to similar levels. The frequency of emotional distractors therefore modified participants ability to effectively control their attention.

\section{The Divide Between Experience and Expectation}


In both Grimshaw et al. (2018) and Schmidts et al. (2020), improved attentional control was suggested to be the result of proactive control engagement, induced by increased expectation of distraction, in line with the DMC framework (Braver, 2012). Recently, however, expectation-based explanations for frequency effects have been questioned. An alternative explanation is that it is the experience of conflict that drives proactive control implementation. As we are exposed to an environment, we develop some history of interaction with sources of conflict. A selection history account (Theeuwes, 2019) suggests that the previous outcomes of selective attention can bias the competition between stimuli for attentional resources. Similarly, others argue that frequent online adjustments in cognitive control may emerge from previous experience with conflict (e.g. Melara \& Algom, 2003). Experience may then bias us to adopt a more proactive control style independently of expectation of upcoming conflict.

The concern for studies manipulating conflict frequency is that experience and expectation are usually confounded. Greater frequency of conflict (either at the response or perceptual level) affords more exposure to conflict while also supposedly building expectation. Studies seeking evidence for an expectation-based account of improved cognitive control must therefore increase the expectation of upcoming conflict without altering the frequency with which conflict occurs.

Cueing $^{2}$ upcoming conflict-present trials makes the conflicting event predictable, increasing expectation without also modifying a participants' experience with conflict. An early example is provided by Logan and Zbrodoff (1982), who applied a purely expectancybased approach by cueing upcoming conflict. Participants were asked to identify whether the word "ABOVE" or "BELOW" was presented above or below a fixation point. A pre-trial cue (word/location) truthfully indicated whether the location of the word was congruent (e.g. the cue ABOVE/above) or incongruent (e.g. the cue ABOVE/below) with its meaning. Consistent with an expectation-based account of control, participants produced faster RTs on a conflicting trial when conflict was cued as opposed to when they were unaware of an upcoming conflict.

An array of more recent studies have incorporated cues into traditional cognitive control paradigms. Bugg and Smallwood (2016) presented pre-trial cues to participants in a four-choice Stroop task ${ }^{3}$ at varying cue-to-stimulus intervals. Participants also completed uninformative trial blocks during which a sequence of nine Xs were presented in place of a cue. Critically, the frequency of incongruent trials was held at $50 \%$ on each block of trials, equating the

\footnotetext{
${ }^{2}$ Note that "cues" here refers only to cues of upcoming presence of conflict. The present arguments are therefore not concerned with spatial cuing or temporal cueing.

${ }^{3}$ Four words each appearing equally often in each of the four colours.
} 
experience of conflict across the cued and uncued blocks. RTs were faster when participants were given pre-trial cues and were able to predict upcoming trial type, with a stronger effect of cues for congruent than incongruent trials. The benefit of cueing was found to be significant for congruent trials across all cue-to-stimulus intervals (500, 1500, $2000 \mathrm{~ms})$. For incongruent trials, a pre-cue benefit (the difference between RTs of cued and uncued trials) occurred only when a 2000 ms cue-to-stimulus interval was used. The key finding here is that making incongruent trials predictable (increasing expectation) improved participants' ability to ignore task-irrelevant information (the meaning of the word) and focus on the task relevant information (print colour), but only when given adequate time to prepare.

A similar effect in a Stroop-like task was reported by Aarts and Roelofs (2010) who also included invalid and neutral pre-trial cues. RTs to validly cued incongruent trials were faster, and invalidly cued trials slower, relative to the neutrally cued trials. The same benefit of cues held for congruent trials. Therefore, valid cues not only allowed participants to anticipate upcoming conflict but also worsened performance when expectations were contradicted. Outside the realm of Stroop-like tasks, expectation-based cognitive control, via explicit cueing, has also been reported in other response conflict tasks such as the flanker task (Correa et al., 2008), and task switching paradigms (Dreisbach et al., 2002; Monsell et al., 2003).

Evidence of expectation-driven control is however not limited to tasks which induce response conflict. Using a task which induces perceptual conflict, Havlíček et al. (2019) presented participants with a search array containing one task-relevant item (a square with a corner missing containing either a vertical or horizontal line) amidst identically coloured complete squares and one bright distractor square. Participants were then asked to report the orientation of the line within the target. A pre-trial cue was presented on some trials which indicated that a distractor would be present (and whether it was going to be presented to the left or right of fixation) or that no distractor would appear. Participant accuracy was found to increase when a distractor was cued as opposed to when it was not. Multiple paradigms, including both response and perceptual conflict-based tasks, therefore show evidence of a control benefit when conflict is expected.

Evidence has also been found for an expectation benefit without explicit cueing (Jiménez et al., 2019) suggesting that cues themselves are not simply modifying control settings regardless of expectation of upcoming conflict. In task-switching studies for example, participants are given two experimental tasks with each trial requiring a response specific to one of these tasks. Conflict occurs between the responses to the different task sets, with participants needing to select the correct response while responses to tasks are inhibited. The 
upcoming task is typically cued to indicate which response is required (Braver et al., 2003). However, in the alternate run task (Rogers \& Monsell, 1995), task switches occur in predictable sequence (once every fixed number of trials) without explicit cues (Andreadis \& Quinlan, 2010; Koch, 2003; Kray \& Lindenberger, 2000). Switch cost (the RT difference between switched task and repeated task trials) is still reduced in this paradigm when sequences of trials are predictable with participants relying only on the sequence of trials to build expectation (Monsell et al., 2003).

The above studies provide evidence that proactive control can be driven by expectation. However, there are studies which show that cues do not always benefit control. An example is Bugg et al. (2015), who compared the separable effects of cues and conflict frequency on Stroop task performance. In their first experiment, participants were shown either mostly incongruent trials (high conflict frequency) or mostly congruent trials (low conflict frequency) in sets of 10. Prior to some trial sets, participants were given valid proportion pre-cues informing them of upcoming conflict frequency (e.g. $80 \%$ matching or $80 \%$ conflicting). For other trial sets participants instead had to rely on acquired experience within the trial set (e.g. no cues were provided).

The Stroop effect was reduced when conflict was frequent as opposed to infrequent (i.e. the typical proportion congruency effect) regardless of whether trial sets were cued. However, the Stroop effect was amplified, rather than reduced, when the mostly congruent trials were cued suggesting that the effect of conflict in the incongruent trials was increased by cueing. In the mostly incongruent blocks of trials, cues did not modify the Stroop effect. The same pattern was replicated in their second experiment, which also indicated that cues did not modify the Stroop effect in a 50\% congruence condition. Furthermore, in the same replication, the RT to the first trial in block was found to be improved by a pre-block cue only when trials were mostly congruent (i.e. not in the $50 \%$ or $80 \%$ conditions). In further replications (Experiment 4) cues led to a reduced Stroop effect in mostly incongruent trial blocks but only on the first trial of a block and only when adequate incentive was provided for participants to make use of them. In all other regards, cues had no effect above that which would be expected given the differences in frequency between groups.

Using pre-trial, rather than pre-block cues, Goldfarb and Henik (2013) presented participants with neutral ${ }^{4}$ or incongruent Stroop trials in equal proportion, following a valid pre-trial cue or no pre-trial cue. The difference between cued and uncued trials (eight-

\footnotetext{
${ }^{4}$ A Hebrew letter string was presented as opposed to a traditional word/colour Stroop trial.
} 
millisecond cueing benefit) failed to reach significance, failing to find benefit to cueing upcoming conflict. In their second experiment, Goldfarb and Henik (2013) also manipulated the relative frequency of incongruent and neutral trials alongside the cue conditions. In this case a significant cue benefit was found only when trials were cued and incongruent trials were infrequent. Hence, Goldfarb and Henik (2013) only observed a cue benefit when proactive control was not already likely engaged based on frequency. Similarly, Bugg et al. (2015) only found a cue benefit when participants were adequately motivated to make use of the foreknowledge of conflict (see also Marini et al., 2015; for similar findings in a perceptual conflict task). Other studies find that timing of cue presentation may also be influential since the effect of valid pre-trial cues was only present in Bugg and Smallwood (2016) on one cueto-stimulus interval (see also; Logan and Zbrodoff, 1982). Amongst the evidence in favour of expectation-driven proactive control, caveats and moderating factors therefore suggest that cognitive control may not be reliant on expectation alone.

This discussion has so far only considered how expectation and experience are contrasted in traditional cognitive control paradigms involving non emotional task-irrelevant stimuli. A smaller body of studies has used explicit expectation-inducing cues to improve the avoidance of emotional distractors. In Augst et al. (2014) (Experiment 2 and 3), participants were asked to ignore a central task-irrelevant distractor (negative, positive, or neutral) and judge if two peripheral flanking lines were parallel. In an informative condition, a cue for distractor valence was provided prior to the presentation of the task array. In an uninformative condition, a nonword cue was used instead, providing no advance information. Distractors of each valence occurred with equal frequency, meaning that most trials contained an emotional distractor. The presence of negative and positive distractors led to greater distraction than neutral distractors in both conditions, i.e. emotional distraction was observed. What was unexpected, was that cues (in the informative condition) did not reduce emotional distraction nor distraction overall indicating no benefit from increased expectation of conflict.

In a more recent study (Schmidts et al., 2020; as described above) also manipulated expectation while presenting participants with task-irrelevant distractors. Participants were presented with a letter sorting task (does a letter follow or precede $\mathrm{m}$ in the alphabet) alongside either negative or neutral images. Trial blocks were mixed, presenting both negative and neutral distractors. Neutral trials were however presented in the majority trials, meaning emotional distractor frequency was low. Cues preceded all trials, informing participants of the valence of the upcoming image, or providing no advance information. In support of an emotional distraction effect, participants were more distracted on trials with negative as opposed to 
neutral distractors. Additionally, in support of experience rather than expectation-driven proactive control, no substantial differences were observed between cued trial RTs and uncued trial RTs for emotional distractors. In fact, Schmidts and colleagues' first and third experiments show a paradoxical increase in distraction by negative stimuli when they were cued. The authors concluded that pre-trial cues did not reduce emotional distraction, regardless of distractor frequency.

A similar valence-specific paradoxical expectation effect was reported by Kleinsorge, (2007). Participants were asked to indicate whether an arithmetic problem, overlaid on the central distractors had been correctly solved. Trials were pre-cued by the colour of a countdown (from 5 to 1) occurring prior to display onset. In a "with anticipation" condition, a green countdown was followed by neutral distractors and a red countdown was followed by negative (Experiment 1a) or positive (Experiment 1b) distractors. In a "without anticipation" condition, countdown colour did not relate to the subsequent trial valence. Importantly, neutral and valenced distractors occurred equally often, meaning there was equal experience of both neutral and emotional distractors. On uncued trials, there was no difference in RT to the arithmetic task between image types. Cued trials with neutral distractors also produced similar levels of distraction to the uncued trials. Cued trials with negative distractors, however, were significantly slower than those with cued neutral distractors. Conversely, RTs were increased when positive distractors were cued, indicating that the paradoxical expectation effect may be specific to the cueing of negative stimuli.

The lack of evidence for cue benefits combined with the existence of paradoxical expectation effects suggests that expectation of upcoming emotional distractors does not necessarily elicit proactive control of emotional distractors. Frequency manipulations in emotional distraction studies do, however, still show improved control when distractors are frequent as opposed to infrequent. In contrast to studies which employ response-conflict tasks or perceptual-conflict tasks using only neutral stimuli, expectation of emotional (and possibly neutral) distractors may not encourage the implementation of proactive control.

\section{Summary}

In sum, emotional stimuli are effective distractors due to their inherent salience as environmental indicators of reward and threat. As per the DMC framework, proactive and reactive control mechanisms aid in ignoring task-irrelevant emotional distractors. The relative use of either type of mechanism is dependent on a contextual cost-benefit analysis. Frequency effects have been found indicating that cognitive control can be biased toward effective control mechanisms, when emotional and neutral distractors occur frequently. Much of existing 
literature supports and claims that the effect of frequency is due to increasing expectation of upcoming distractors; thought to bias control to a more proactive style.

A contest to an expectation-based explanation for frequency effects is that experience of conflict varies alongside frequency of conflict. While previous studies find evidence in favour of expectation-driven proactive control, there are those which find that expectation driven proactive control may be reliant on additional factors such as motivation/reward and cue to stimulus timing (Bugg \& Smallwood, 2016; Bugg et al., 2015; Goldfarb \& Henik, 2013; Logan \& Zbrodoff, 1982; Marini et al., 2015). Few emotional distraction studies have manipulated expectation independently of frequency manipulations and those which attempt to manipulate expectation find no evidence for expectation-based proactive control (Augst et al., 2014; Kleinsorge, 2007; Schmidts et al., 2020). Indeed, these studies indicate that expectation may paradoxically increase rather than decrease distraction.

\section{The Present Experiments}

We are therefore left at a crossroads in explaining whether expectation or experience of distraction drives control of attention, specifically in the avoidance of emotional distraction. To clarify the driving factors behind the effects of frequency manipulations on emotional distraction, I investigated the extent to which the expectation of distractor presence can reduce distraction by both neutral and emotional task-irrelevant distractors. I conducted two experiments to assess control of attention when infrequent neutral and salient emotional distractors are expected as compared to when they are not. Modified versions of the emotional irrelevant-distractor paradigm (Grimshaw et al., 2018) were used for both experiments.

The emotional distraction paradigm was used because this paradigm has reliably induced emotional distraction in previous studies (Grimshaw et al., 2018, Grimshaw et al., 2020). Additionally, distractors are not present on all trials, meaning distraction (the difference in RT owed to the presence versus the absence of a distractor) by neutral and emotional distractors can be assessed independently. This direct assessment of distraction is not possible in previous emotional distraction studies which manipulate the relative proportion of neutral and emotional distractor-present trials (e.g. Schmidts et al., 2020).

The present experiments also implemented a novel manipulation of expectation and did not cue upcoming distractors with pre-trial cues. Cue-to-stimulus interval timing, motivation, and participant instructions have all been shown to alter the benefit of conflict cues on task performance (Bugg \& Smallwood, 2016; Bugg et al., 2015; Chiew \& Braver, 2016; Goldfarb \& Henik, 2013; Marini et al., 2015). Cue benefits have also been inconsistent in previous cognitive control studies, especially when concerning emotional distraction. Particularly in 
studies which employ task-irrelevant distractors, the cue itself may also alter responses to the upcoming distractors due to priming rather than effortful cognitive control (Theeuwes, 2013), possibly evidenced by paradoxical expectation effects in previous studies.

Another method to building expectation is suggested by the alternate run task-switching procedure which bypasses the problems with cue-based studies and instead builds an implicit expectation of upcoming distractor presence via more naturalistic methods than pre-trial cueing (Egner \& Hirsch, 2005). Predictable sequential conflicts have been shown to improve control relative to conflict that occurs randomly (Andreadis \& Quinlan, 2010; Koch, 2003; Kray \& Lindenberger, 2000). Presenting distractors in a predictable sequence also provides the benefit of cues in that minimal modification to the emotional irrelevant-distractor paradigm is required. Since only trial order is altered, frequency can be held constant between conditions, equating experience, but also allowing the present study to be comparable to previous studies such as in which only frequency was manipulated (Grimshaw et al., 2020; Grimshaw et al., 2018). Consequently, I manipulated expectation in the present experiments by presenting distractors either predictably (in sequence) on every fourth trial, or randomly on an equal proportion of trials $(25 \%)$.

In Experiment 1, peripheral task-irrelevant images were presented alongside a simple perceptual task. Distractors were negative (gore and mutilation) or neutral (people doing everyday activities) scenes. These distractors occurred either predictably (i.e. every fourth trial, allowing for expectation of upcoming distractors) or unexpectedly but equally often $(25 \%$ of trials). Distraction was measured as the difference between trials in which a distractor was present and trials in which they were not. Experiment 2 was a conceptual replication of the first with a few key differences. The same distractors were presented at fixation with the same predictability manipulation. An additional high distractor frequency condition, in which distractors on a random $75 \%$ of trials, was also added to compare the effects of expectation to a high-experience condition which has been shown to produce good control in previous frequency manipulations. Participants also completed a post-experiment questionnaire assessing their awareness of the predictability and frequency of distractor occurrence, and whether they engaged effortful and voluntary control strategies.

\section{Experiment 1}

Experiment 1 investigates whether expectation drives proactive control implementation when experience of distractors is held constant. To do this, a modified version of the emotional irrelevant-distractor paradigm was used to expose participants to equal frequency of distractors while manipulating expectation. 
Participants were asked to complete a simple letter identification task (identify whether a central letter array contains a $\mathrm{K}$ or $\mathrm{N}$ ) while emotional (high arousal negative) and neutral images from the International Affective Picture System (IAPS) (Lang et al., 2008) were presented in the periphery on some trials. To create a context wherein images were distracting, and participants are thought to rely on reactive control, distractor frequency was low (25\% of trials). Distractor frequency was the same for all participants to maintain equal overall experience of distractors. To differentiate expectation of upcoming distractors without pre-trial cues, distractors occurred on every fourth trial for half of the participants (the predictable condition) or on a random $25 \%$ of trials for the other half (the unpredictable condition).

I measured the time taken to respond to the letter identification task (RT) and accuracy of task performance. Because the main hypothesis concerns distraction rather than simply RT, an index of distraction was also calculated by subtracting participants' averaged distractorabsent trial RT from the averaged distractor-present trial RT for each valence of distractor.

Ample evidence suggests that low distractor frequency biases control mechanisms towards less effective strategies (Bugg et al., 2008; Gonthier et al., 2016; Grimshaw et al., 2018). Therefore, it was expected that the distractor images would compete effectively for attentional resources when unpredictable. Moreover, due to the bias to attend to emotional stimuli, negative distractors were expected to elicit greater distraction than neutral stimuli when both frequency and expectation of distractors are low (i.e. the unpredictable low-frequency condition). Previous studies (e.g. Grimshaw et al., 2018) have indicated that, when distractors are frequent, participants can avoid emotional distractors just as well as they avoid neutral distractors. Thus, emotional distraction (the difference in distraction by negative distractors and neutral distractors) is predicted to be lesser when emotional distractors are predictable.

If expectation of upcoming distractors drives proactive control, then when distractors are infrequent yet predictable (i.e. expected), proactive control should be encouraged. Thus, participants were predicted to be less distracted when distractors were predictable as opposed to unpredictable ${ }^{5}$. Conversely, if expectation alone cannot drive participants to implement proactive control, then distraction should not differ between the predictable and unpredictable conditions.

\section{Method}

\section{Participants}

\footnotetext{
${ }^{5}$ Reflected in an interaction effect between Distractor presence and Predictability of distractors. This interaction includes distractor presence to capture the change to RT caused by a distractors presence as relative to its absence (i.e. distraction).
} 
Participants were 104 female $^{6}$, first-year undergraduate students (aged 18 to 32, $M=$ $18.69, S D=2.35)$. All participants had normal or corrected to normal vision and were not currently taking medication for depression or anxiety disorders. All participants provided written informed consent and received credits for a research participation component of their first-year studies. This study received approval from the Human Ethics Committee of the School of Psychology, Victoria University of Wellington, New Zealand.

\section{Sample Size Determination}

There was no applicable pilot study nor comparable previous study using peripheral distractors in quadrants. Hence to detect the predicted Predictability $\times$ Valence interaction, an estimated moderate effect size was used in an a priori power analysis. G-power analysis indicated that, to achieve a moderately sized interaction effect, (Cohen's $\mathrm{f}=.25)\left(\eta_{p}^{2}=.06\right)$, a sample size of 98 participants would be needed to attain a power of $80 \%$. For counterbalancing purposes, the total sample size was increased to 104 participants (52 in each predictability condition). Three participants who did not provide demographic information and a further two participants who did not meet eligibility criteria were replaced.

\section{Stimuli}

Twelve neutral and 12 negative images (occupying $12^{\circ} \times 12^{\circ}$ of visual angle) from the IAPS database (Lang et al., 2008), were used. Ratings of arousal and valence of the images were obtained from female norms using the nine-point Self-assessment Manikin rating scale reported in Lang et al. (2008). The neutral images were scenes of people performing everyday activities, rated near the midpoint of the valence scale $(M=5.01, S D=.34)$ and low arousal $(M$ $=3.07, S D=.32)$. Negative images were scenes of gore and mutilation, rated as lower (negative) in valence $(M=1.64, S D=.21)$ and higher in arousal $(M=6.53, S D=.42)$. Six additional images of each valence were used in the practice trials. All images were presented in colour on a black background and were matched for luminance and contrast using the MATLAB, SHINE toolbox (Willenbockel et al., 2010). Appendix A shows a list of the IAPS images and their individual valence and arousal ratings.

The letter array consisted of 5 lower case o's (occupying $.22^{\circ}$ by $.22^{\circ}$ of visual angle each) and one uppercase $\mathrm{N}$ or K (occupying $.69^{\circ}$ by $.69^{\circ}$ of visual angle), presented in white Arial font, arranged vertically along the midline of the screen. The letters were all separated by

\footnotetext{
${ }^{6}$ Differences in valence and arousal assessments of the negative IAPS images have previously been found between male and female samples (Lang et al., 2008). Given this difference, the present experiment used only female participants to ensure that that the interpretations of the images were more likely to be consistent between participants. The valence and arousal ratings presented in Appendix A represent ratings by an exclusively female sample.
} 
$.69^{\circ}$ of visual angle with the top and bottom letters of the array positioned $1.75^{\circ}$ of visual angle from the centre of the screen. An example of the task array including a distractor is shown in Figure 1A.

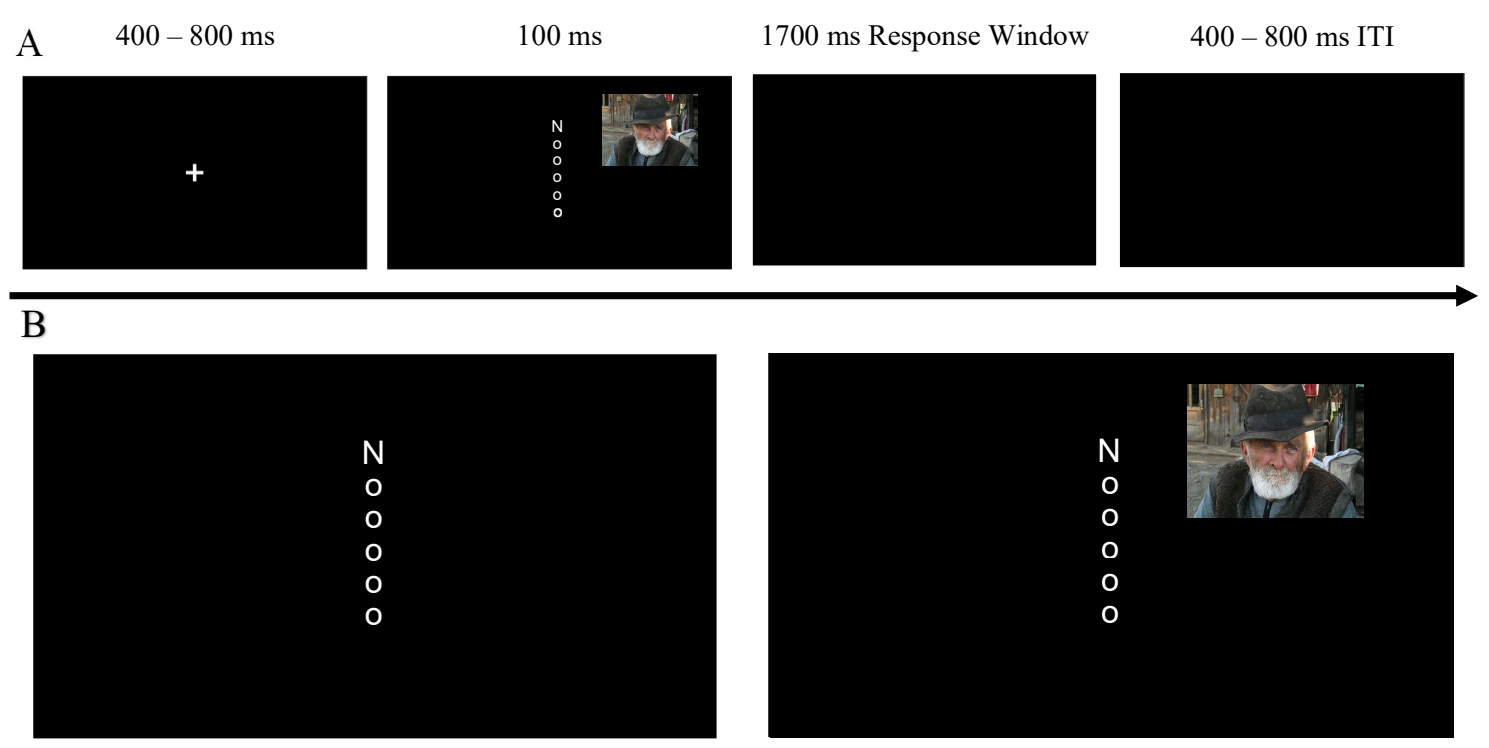

Figure 1. A) Trial structure of Experiment 1, showing a neutral distractor-present trial B) Examples of a distractor-absent trial (left) and a neutral distractor-present trial (right)

\section{Procedure}

Participants were seated in a walled cubicle in a dimly lit room, $57 \mathrm{~cm}$ from a computer monitor (24', $1920 \times 1080$-pixel, AOC monitor with a refresh rate of $120 \mathrm{~Hz}$ ), connected to a Dell T1700 computer. Psychopy 3 software (Peirce et al., 2019) was used for stimulus presentation and response recording. Viewing distance was maintained by a chinrest.

A trial began with the presentation of a central fixation cross (occupying $.84^{\circ} \times .84^{\circ}$ of visual angle) lasting between 400 and $800 \mathrm{~ms}$. Participants were then shown an array of 6 letters arranged vertically down the midline of the screen, for $100 \mathrm{~ms}$. Participants were asked to respond with a button press (using the index and middle fingers on their dominant hand on the 1 and 2 keys of the numpad) to indicate whether the letter $\mathrm{K}$ or $\mathrm{N}$ was present in the letter array. The button-response mapping was counterbalanced across participants. Within a block of trials, each target letter ( $\mathrm{K}$ or $\mathrm{N}$ ) occurred on a random half of trials and appeared in each of the six array locations four times.

Participants could respond within an $1800 \mathrm{~ms}$ window from array onset. On participant response, the trial proceeded to a variable inter trial interval (ITI) equal to the duration of 
fixation cross. On an incorrect response, or if no response was made, participants received auditory feedback (a $100 \mathrm{~ms}$ long tone), which they were told was indicative of an incorrect response. The dependent variables of interest were participant reaction time (RT) from stimulus array onset and their accuracy on the letter task.

Participants were assigned to either the predictable condition, wherein distractors occurred on every fourth trial of the experiment (25\% of trials), or to the unpredictable condition, wherein distractors occurred on a random $25 \%$ of trials. On distractor-present trials, a single image (neutral or negative) was present alongside the letter array. Images were presented alongside the letter array in one of four quadrants, with the centre of the image located $7.4^{\circ}$ (horizontally) and $6.8^{\circ}$ (vertically) from the centre of the screen, as shown in Figure 1B. Images appeared an equal number of times in each quadrant.

The experiment consisted of four blocks of 48 trials. Blocks were separated by a 30 second minimum break. Valence was blocked so that all the distractors in a block were of the same valence. Half the participants in each condition completed the blocks in a Neutral, Negative, Negative, Neutral order, and half did so in the inverse Negative, Neutral, Neutral, Negative order.

Participants completed two 24-trial practice blocks. The first contained only neutral distractors and the second contained only negative. Distractors occurred on every fourth practice trial for those in the predictable condition, while they occurred on a random six trials $(25 \%)$ for those in the unpredictable condition. To make participants aware of the order of distractor-present and absent trials in the experiment, pre-experiment instructions stated that an image would be present "on every fourth trial" (the predictable condition) or on "some" trials (the unpredictable condition). This information was presented both through on-screen instructions and verbally, prior to both practice trials and experimental trials.

\section{Design}

This experiment employed a mixed design with Predictability (Predictable or Unpredictable) manipulated between subjects, and Valence (Neutral or Negative), and Distractor presence (Present or Absent) manipulated within subjects. The dependent variables were RT (ms) and accuracy (\% correct responses to the letter task). Additionally, a distraction index was calculated for each valence by subtracting the RT for distractor-absent trials from the RT of distractor-present trials.

\section{Statistical Analysis}

RT analyses were conducted only on trials wherein participants responded correctly, and RT was greater than $200 \mathrm{~ms}$, excluding $8.25 \%$ of trials. Additionally, to ensure that 
participants were engaged in the task, an exclusion criterion was established which removed participants with lower than $70 \%$ accuracy on blocks of either valence, or accuracy lower than $75 \%$ overall. No participants met these criteria. Greenhouse-Geisser corrections were performed where necessary on the primary analyses. Effect sizes were calculated for all inferential statistics. Effect sizes are presented in partial eta squared $\left(\eta_{p}{ }^{2}\right)$ for main effects and interactions and in Cohen's $d_{z}$ for within subject comparisons of means (Lakens, 2013).

\section{Results}

RT

Table 1 presents the mean RTs, standard deviations and distraction indices by Predictability, Valence, and Distractor presence. To provide a clear assessment of distraction by images of each valence within each condition, separate paired samples t-tests were conducted comparing distractor-absent to distractor-present trials. T-tests (Table 1) indicate that distraction is present for each valence of distractor within each condition, showing that distractors were able to outcompete task relevant stimuli for attentional resources.

To assess whether Predictability or Valence modified distraction, mean RTs (Figure 2) were entered into a 2 (Distractor presence: Present, Absent) $\times 2$ (Valence: Neutral, Negative) $\times 2$ (Predictability: Predictable, Unpredictable) mixed ANOVA. The main effects of Distractor presence, $F(1,102)=50.22, p<.001, \eta_{p}^{2}=.33$, and Valence, $F(1,102)=5.08, p=.026, \eta_{p}^{2}=$ .05 , were qualified by a significant Valence $\times$ Distractor presence interaction, $F(1,102)=12.28$, $p<.001, \eta_{p}{ }^{2}=.11$. Follow-up t-tests showed that participants were slower when distractors were present for both neutral, $t(103)=4.22, p<.001, d_{z}=0.41$, and negative, $t(103)=6.54, p$ $<.001 d_{z}=0.64$, distractors. However, the interaction reflects greater distraction by negative $(M=27, S D=43)$ as opposed to the neutral $(M=12, S D=29)$ distractors. Hence, there is support for the prediction that RTs would be greater when distractors were present (a distraction effect) and more so when distractors were negative than if they were neutral (an emotional distraction effect).

No main effect of Predictability was found, $F(1,102)=0.45, p=.502, \eta_{p}{ }^{2}=.004$, indicating no overall difference in RT between conditions. However, a significant Predictability $\times$ Distractor presence interaction, $F(1,102)=4.19, p=.043, \eta_{p}{ }^{2}=.04$, was observed. Follow-up paired samples t-tests were conducted to assess whether this interaction reflects lower distraction in the predictable relative to the unpredictable condition, as predicted. While participants were slower on distractor-present as opposed to distractor-absent trials in both the predictable, $t(51)=5.74, p<.001, d_{z}=0.80$, and unpredictable conditions, $t(51)=$ $4.16, p<.001, d_{z}=0.58$, this difference was larger in the predictable condition $(M=25, S D=$ 
40) than the unpredictable condition $(M=14, S D=33)$. Results therefore indicate that distraction (the difference between distractor-present and distractor-absent RT) was not just present in both conditions but that is was indeed greater in the predictable condition. Additional analyses focussed on the distraction indices (included in Appendix B), indicated that distraction was in fact significantly greater in the predictable condition $(M=25, S D=40)$ relative to the unpredictable condition $(M=14, S D=33)$, reflecting the same interaction effect as above, $F(1$, 102) $=4.19, p=.043, \eta_{p}^{2}=.04$.

In sum the RT analyses indicate that substantial distraction was present in both the unpredictable and predictable conditions. Additionally, emotional stimuli proved to be more distracting than neutral stimuli regardless of distractor predictability. Counter to predictions, when participants could expect upcoming infrequent distractors, distraction was greater than if participants had no expectation.

Table 1

Mean (SD) RTs (in ms), Distraction indices and paired samples t-test results, including confidence intervals and effect sizes (Cohen's $d_{z}$ ), by Valence, Predictability and Distractor presence in Experiment 1

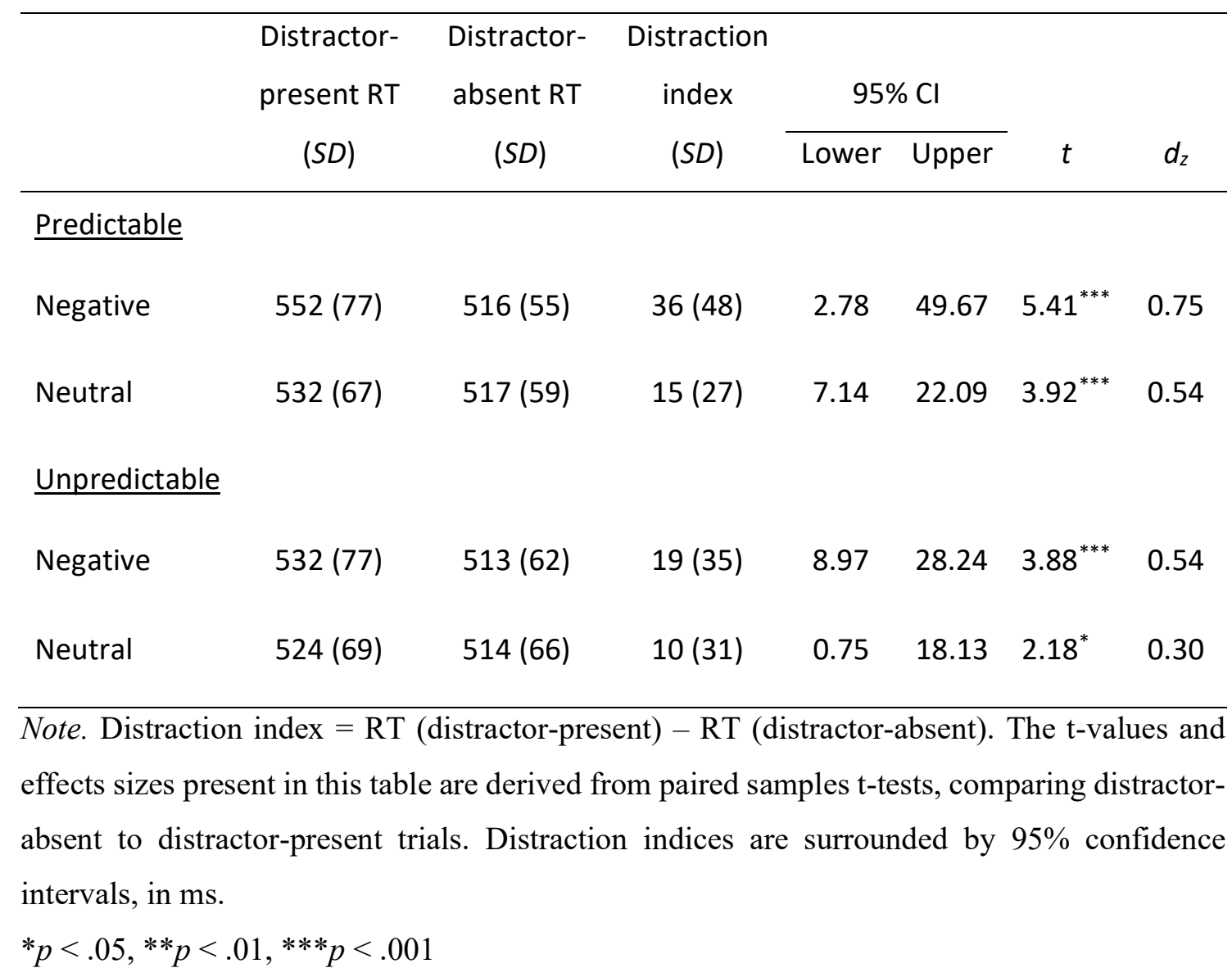




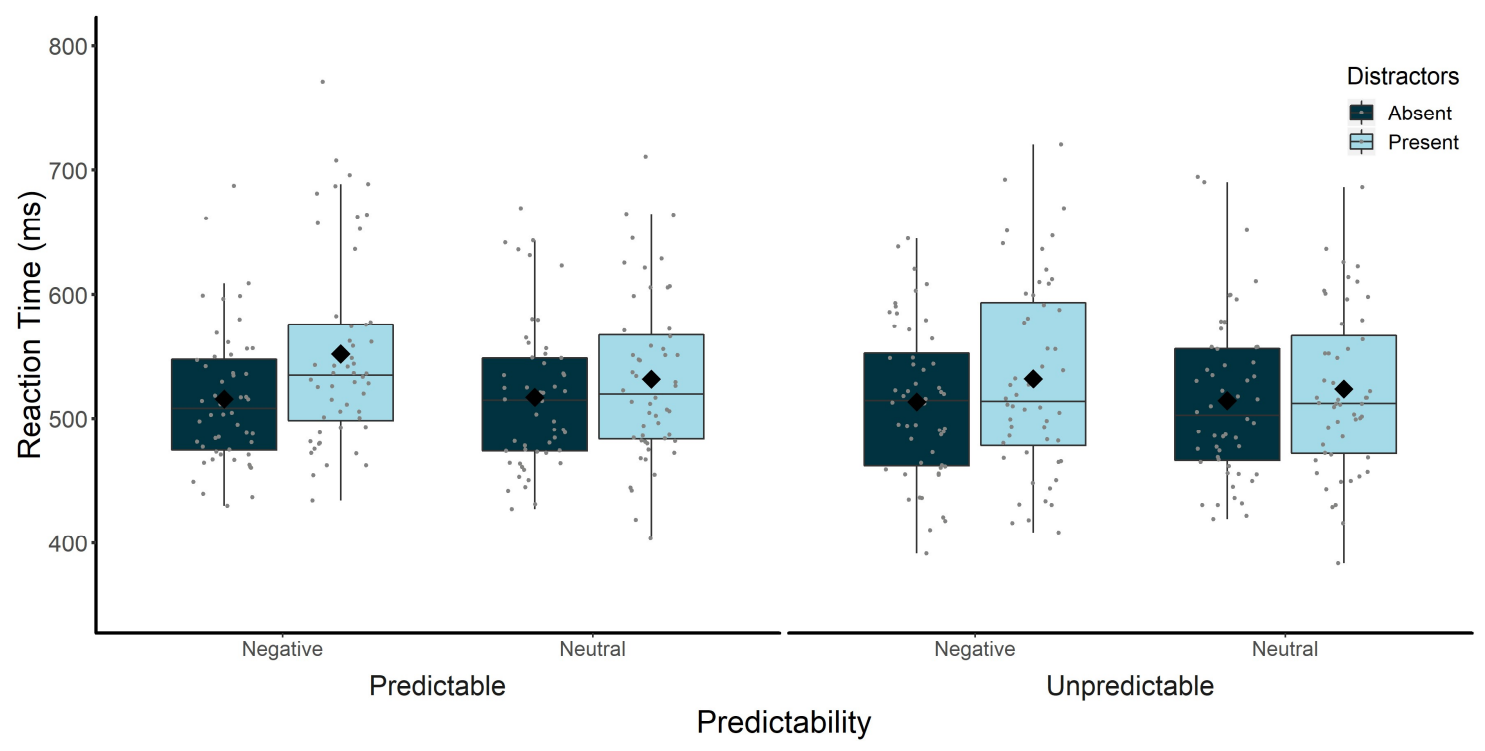

Figure 2. Experiment 1 individual RTs, by Predictability, Valence and Distractor presence Note. Diamonds indicate mean RT. Boxes contain the middle quartiles and the bar indicates median RT.

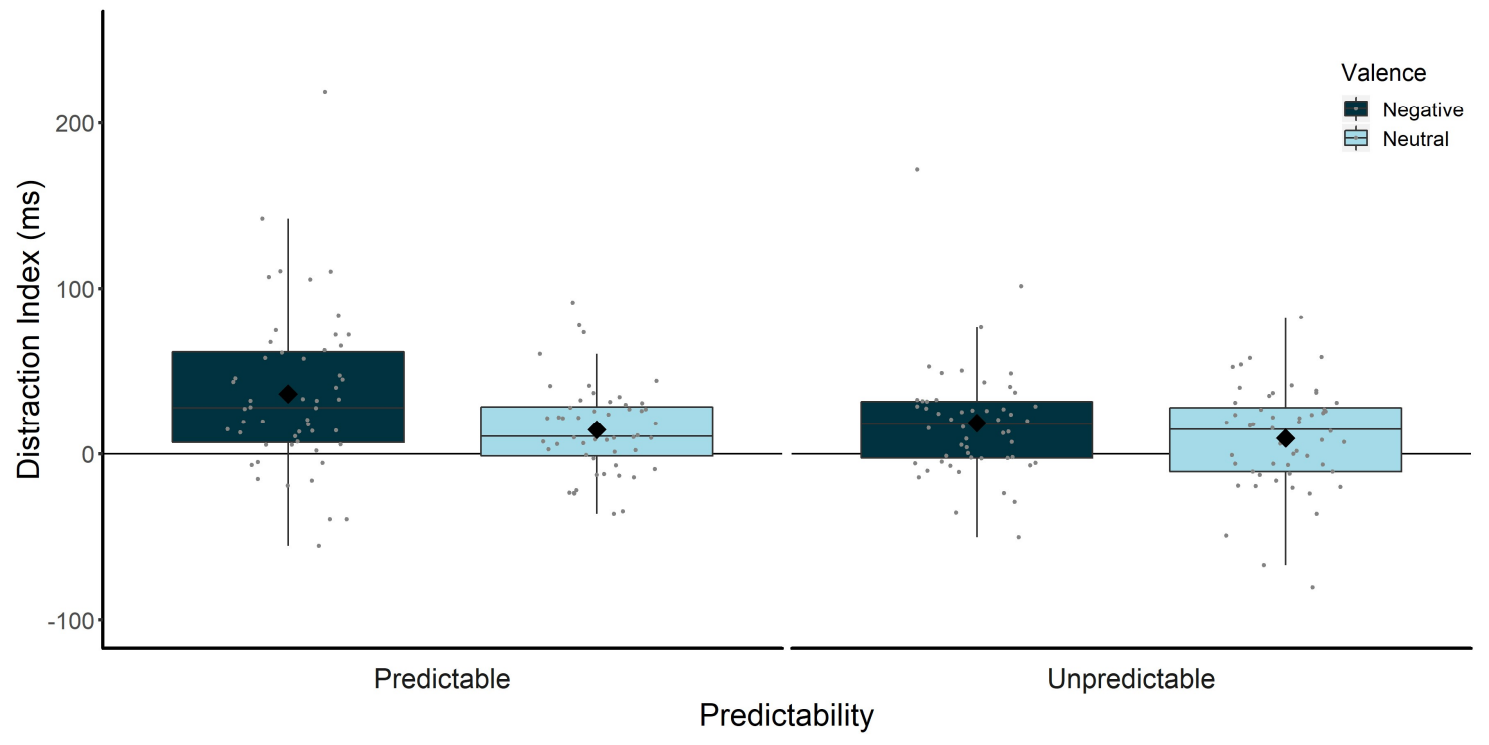

Figure 3. Experiment 1 individual Distraction indices by Valence and Predictability Note. Diamonds indicate mean Distraction index. Boxes contain the middle quartiles and the bar indicates median Distraction index. 


\section{Accuracy}

Participant's mean accuracy (as a percentage), by Predictability, Valence and Distractor presence are presented in Table 2. Accuracy was high overall, with participants only making an average of 11.22 errors $(S D=8.48)$ throughout the experiment $(5.84 \%$ of trials $)$. A possible explanation of the RT results is that participants strategically slowed responding on distractor present trials to increase the accuracy of their responses. To assess whether there was such a speed-accuracy trade off, accuracy scores were entered into a 2 (Distractor presence: Present, Absent) $\times 2$ (Valence: Neutral, Negative) $\times 2$ (Predictability: Predictable, Unpredictable) mixed ANOVA. A main effect of condition was observed, $F(1,102)=4.08, p=.046, \eta_{p}^{2}=.04$, with higher accuracy being found in the predictable condition $(M=94.83, S D=4.10)$ than in the unpredictable condition $(M=93.48, S D=5.16)$. No main effects were found for either valence, $F(1,102)=0.64, p=.425, \eta_{p}{ }^{2}=.01$, or distractor presence, $F(1,102)=1.32, p=.253$, $\eta_{p}^{2}=.01$.

Participants in the predictable condition were therefore more accurate overall (Mdifference $=1.35 \%$, equivalent to 2.6 trials difference; see Figure 4). That said, accuracy analysis indicated no interaction between predictability and distractor presence. Thus, a speedaccuracy trade-off is unlikely to be driving the effect of predictability on distraction as there is no evidence to suggest that participants selectively slowed responding to improve accuracy on distractor present trials. 
Table 2

Mean (SD) accuracy scores (in \% correct trials) and paired samples t-test results, including confidence intervals and effect sizes (Cohen's $d_{z}$ ), by Valence, Predictability and Distractor presence in Experiment 1

\begin{tabular}{|c|c|c|c|c|c|c|c|}
\hline & \multirow{2}{*}{$\begin{array}{l}\text { Distractor- } \\
\text { present } \\
(S D)\end{array}$} & \multirow{2}{*}{$\begin{array}{l}\text { Distractor- } \\
\text { absent } \\
\text { (SD) }\end{array}$} & \multirow{2}{*}{$\begin{array}{l}\text { Accuracy } \\
\text { difference } \\
(S D)\end{array}$} & \multicolumn{2}{|c|}{$95 \% \mathrm{Cl}$} & \multirow[b]{2}{*}{$t$} & \multirow[b]{2}{*}{$d_{z}$} \\
\hline & & & & Lower & Upper & & \\
\hline \multicolumn{8}{|c|}{ Predictable } \\
\hline Negative & 95.59 (5.31) & $94.34(4.74)$ & $1.25(5.73)$ & -0.39 & 2.90 & 1.48 & 0.21 \\
\hline Neutral & $95.83(4.74)$ & $94.74(4.44)$ & $1.09(4.73)$ & -0.18 & 2.37 & 1.59 & 0.22 \\
\hline \multicolumn{8}{|c|}{$\underline{\text { Unpredictable }}$} \\
\hline Negative & $92.71(8.16)$ & $93.80(5.67)$ & -1.09 (7.75) & -3.25 & 1.06 & 1.02 & 0.14 \\
\hline Neutral & $93.91(5.80)$ & $93.27(5.17)$ & $0.64(4.82)$ & -0.70 & 1.98 & 0.84 & 0.12 \\
\hline
\end{tabular}

Note. The t-values and effects sizes present in this table are taken from paired samples t-tests, comparing accuracy on distractor-absent to distractor-present trials. Accuracy differences are surrounded by $95 \%$ confidence intervals, in $\%$ correct trials.

${ }^{*} p<.05,{ }^{* *} p<.01,{ }^{* * *} p<.001$

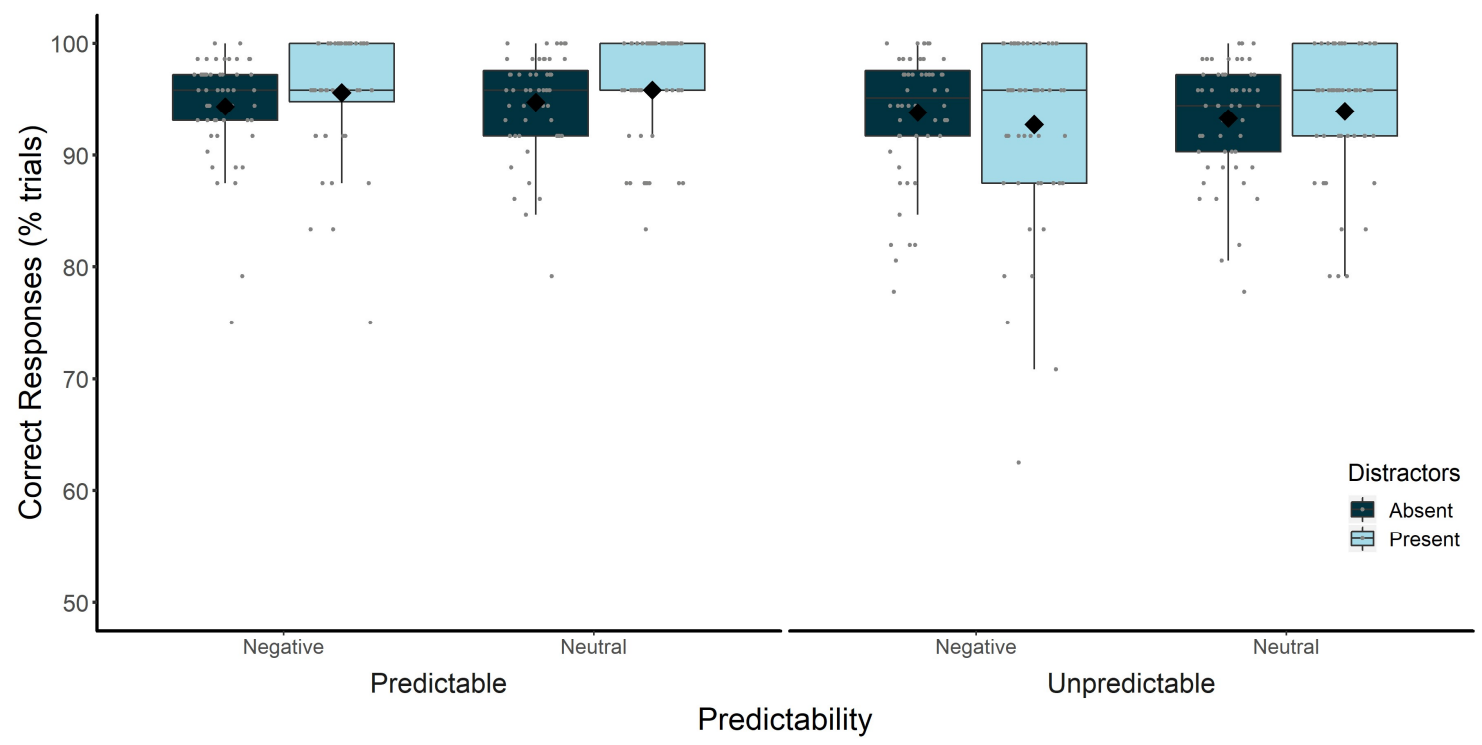

Figure 4. Experiment 1 individual Accuracy scores by Predictability, Valence and Distractor presence

Note. Diamonds indicate mean Accuracy score. Boxes contain the middle quartiles and the bar indicates median Accuracy score. 


\section{Discussion}

Experiment 1 investigated whether expectation of upcoming conflict biases cognitive control of distraction (neutral or emotional) towards proactive strategies. As expected, participant responses were significantly slower when distractors were present, showing that the emotional irrelevant-distractor paradigm effectively elicits distraction. Moreover, distraction by negative distractors was greater than that by neutral distractors, providing evidence of emotional distraction above that of distraction by neutral stimuli.

The primary predictions were that distraction would be overall lower in the predictable as opposed to unpredictable conditions and that distraction would be more reduced for negative as opposed to neutral distractors. In contrast, RT and distraction indices analyses indicated that distraction was greater in the predictable condition rather than the unpredictable condition. Hence, the current findings indicated a paradoxical effect of expectation, in that increased expectation of distractors led to greater rather than lesser distraction.

A concern addressed by the accuracy analysis is the possibility of a speed-accuracy trade off in responding. Since participants in the predictable condition are aware of when distractors will occur, participants could selectively slow RT to improve accuracy on distractorpresent trials. Accuracy was in fact found to be greater in the predictable than the unpredictable condition. However, this was seen in both distractor-present and distractor-absent trials. Hence, it is unlikely that the greater overall distraction observed in the predictable condition reflects a strategic slowing of responses specifically when distractors were expected.

The results of Experiment 1 are counterintuitive. In both the predictable and unpredictable conditions, participants were exposed to distractors on $25 \%$ of trials, occurring in sequence only in the predictable condition. In terms of the DMC framework, it would be expected that increased expectation and ability to prepare for upcoming distractors would bias participants to using proactive control mechanisms. Results instead suggest that participants adopted a reactive as opposed to proactive control mode when distractors could be expected. The present findings therefore indicate that control implementation was not biased by expectation but instead by experience of distraction. Moreover, a paradoxical expectation effect was observed, suggesting that attention to distractors was heightened when they were expected. However, the three way Predictability $\times$ Valence $\times$ Distractor presence interaction did not reach significance indicating that the paradoxical expectation effect was only numerically, but not statistically, greater for negative than for neutral distractors as it was in previous studies (Kleinsorge, 2007;

Schmidts et al., 2020). Whether the paradoxical expectation effect is valence specific, may be 
an important line of future investigation as it could suggest that expected emotional stimuli pose a unique challenge to cognitive control.

The current findings therefore contradict studies, primarily employing response conflict tasks, which find that explicit expectation of upcoming conflict can improve control (Bugg \& Smallwood, 2016; Fernandez-Duque \& Knight, 2008; Logan \& Zbrodoff, 1982). Instead, current findings converge with a collection of studies which suggest that participants adopt a proactive mode of control when experience of conflict is increased regardless of expectation (Bugg et al., 2015; Kleinsorge, 2009; Moher \& Egeth, 2012; Schmidts et al., 2020). Of these, several are studies which employ an irrelevant-distractor paradigm and present emotional distractors (Kleinsorge, 2009; Schmidts et al., 2020). The present study is therefore consistent with the idea that avoidance of emotional distractors is not benefited by expectation of their occurrence.

However, the current study differs slightly in method from previous implementations of the emotional irrelevant-distractor paradigm in that distractors were presented in quadrants as opposed to above and below the letter array as in Grimshaw et al. (2018). Hence, compared to previous uses of the task, distractor location was less predictable. Consequently, distractors may still have been somewhat surprising to participants since they did not know where they would occur, only that they would occur.

The current experiment also did not include a high distractor frequency condition, which has been hypothesised to encourage the use of proactive control (Grimshaw et al., 2018; Schmidts et al., 2020). Without a high frequency condition, there is no "proactive control condition" to compare distraction in the unpredictable low-frequency condition against. Hence the predictable condition (i.e. the effect of expectation which is being investigated) cannot be compared against a typical distractor frequency manipulation.

As a final point, Experiment 1 can also not indicate how, or whether, participants are making use of the predictability of distractors. Braver (2012) suggests that expectation-driven proactive control is voluntary and strategic. In the present experiment, whether participants are deliberately and strategically implementing control based on distractor predictability is unclear but may reflect whether strategic control is being attempted. Insight into how participants are engaging with and experiencing the task is needed to better understand the mechanisms they are using to avoid distraction.

\section{Experiment 2}

A second experiment was conducted to determine if the counterintuitive results of Experiment 1 would replicate, while also modifying the experimental design to address 
methodological concerns. Experiment 2 therefore used the same stimuli and predictability manipulation as Experiment 1 with three changes. 1) To compare the predictable condition to a typical manipulation of distractor frequency, a third condition was added wherein distractors were frequent ( $75 \%$ of trials) and unpredictable. 2 ) To ensure that distractor location as well as their occurrence was predictable with certainty, distractors were no longer presented in quadrants. A recent study in our lab, (Grimshaw et al., 2020), used an emotional irrelevantdistractor paradigm wherein all emotional distractors were presented at fixation. Participants were more distracted at low as opposed to high distractor frequency similar to the frequency effects in previous studies(Grimshaw et al., 2018; Schmidts et al., 2020). Hence, in Experiment 2 distractors were only ever presented centrally (at fixation) with targets appearing above and below. Pixel scrambled images were presented on distractor-absent trials, equating visual stimulation across conditions. 3) A post-experiment questionnaire was included to probe participants' awareness of the predictability manipulation and their strategic control implementation.

Reflecting the distractor frequency effect, distraction was predicted to be lesser in the unpredictable high-frequency condition than in the unpredictable low-frequency condition. Distraction was also predicted to differ more between the negative and neutral trial blocks in the unpredictable low-frequency than in the unpredictable high-frequency condition which would indicate less emotional distraction when distractors occur frequently (i.e. a reduction in the difference in distraction by emotional and neutral stimuli).

Concerning the effect of expectation three competing predictions were investigated. First, if increased expectation drives participants to implement proactive control, then individuals should be less distracted when they can expect upcoming distraction. When distractors are predictable, there should therefore be less distraction than when they occur randomly. Conversely, the second prediction stated that if proactive control relies instead on experience of distractors, then there should be similar distraction when experience is equal regardless of expectation of upcoming distraction. Hence, distraction would be lowest when distractor frequency is high and would not differ when distractors occurred infrequently, regardless of whether they are predictable. A third alternative hypothesis, stemming from the paradoxical expectation effect found in Experiment 1, suggests that expectation of upcoming distractors harms instead of helps our ability to avoid distraction. Distraction should then be greater rather than lesser when distractors are predictable as opposed to random.

\section{Method}

\section{Participants}


Participants were 96 female, first-year undergraduate students (Aged 18 to $30 \mathrm{M}=$ $19.24, S D=2.24)$. All participants had normal or corrected to normal vision and were not currently receiving treatment for depression or anxiety disorders. All participants provided written informed consent and received credits for a research participation component of their first-year studies. One participant withdrew from the study due to discomfort and was replaced. This study received approval from the Human Ethics Committee of the School of Psychology, Victoria University of Wellington (New Zealand).

\section{Sample Size Determination}

In Experiment 1 a moderate effect size was used for a priori power analysis. In Experiment 2 an effect size of Cohen's $\mathrm{f}=.33\left(\eta_{p}{ }^{2}=0.10\right)$ was used for a priori power analysis. This was based on previous in-lab studies (Grimshaw et al., 2020) employing the emotional distractor paradigm, using central distractors and assessing a Frequency $\times$ Valence interaction in RTs and distraction indices. G-power analysis indicated that, to achieve an effect size of Cohen's $\mathrm{f}=.33$, a sample size of 96 participants would be needed to attain a power of $90 \%$.

\section{Stimuli}

The IAPS images used were the same as were used in Experiment 1 (see Appendix A). Images were presented in the centre of the screen and occupied $11^{\circ}$ of visual angle horizontally and $8.25^{\circ}$ vertically. As shown in Figure $5 \mathrm{~B}$, the letter array now consisted of six letters arranged in two rows of three, positioned $4.87^{\circ}$ above and below the midline of the screen and horizontally separated by $5.07^{\circ}$. The letters of the letter array were all presented in white Arial front occupying $0.86^{\circ}$ by $0.92^{\circ}$ of visual angle. Five of the letters in the array were capital Os while a random sixth letter was either a capital $\mathrm{K}$ or $\mathrm{N}$.

For each IAPS image, three pixel-scrambles were made. Each of these scrambled images were of the same size and resolution as the original intact image. Each of the 12 IAPS images were shown once per block in the unpredictable low-frequency and predictable lowfrequency conditions. Three pixel-scrambles of each IAPS image were used for scrambleddistractor trials wherein the pixel scrambles were shown rather than the intact distractor. In the unpredictable high-frequency condition, each intact image was shown three times without consecutive duplicates. On scrambled trials a single scramble of each of the 12 intact images was used. All scrambled images were only ever presented in the same block as their intact counterparts. All images were presented in colour on a black background and were matched for luminance using the MATLAB Shine toolbox. 


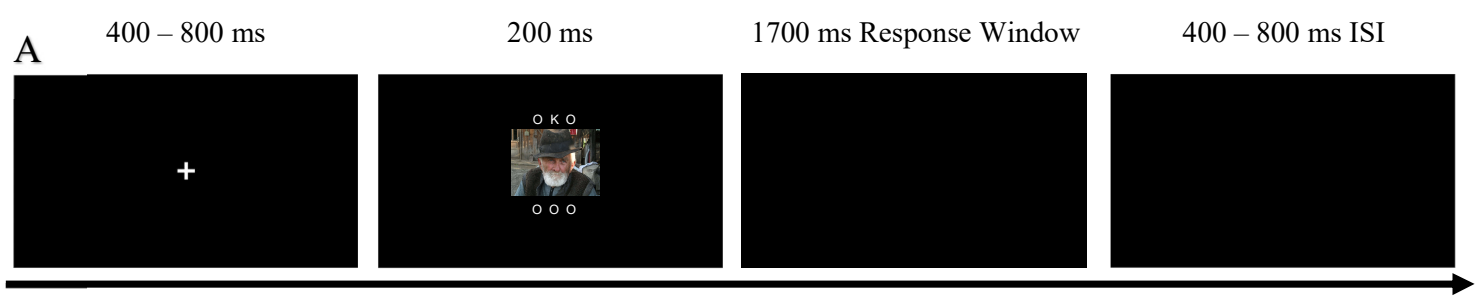

B
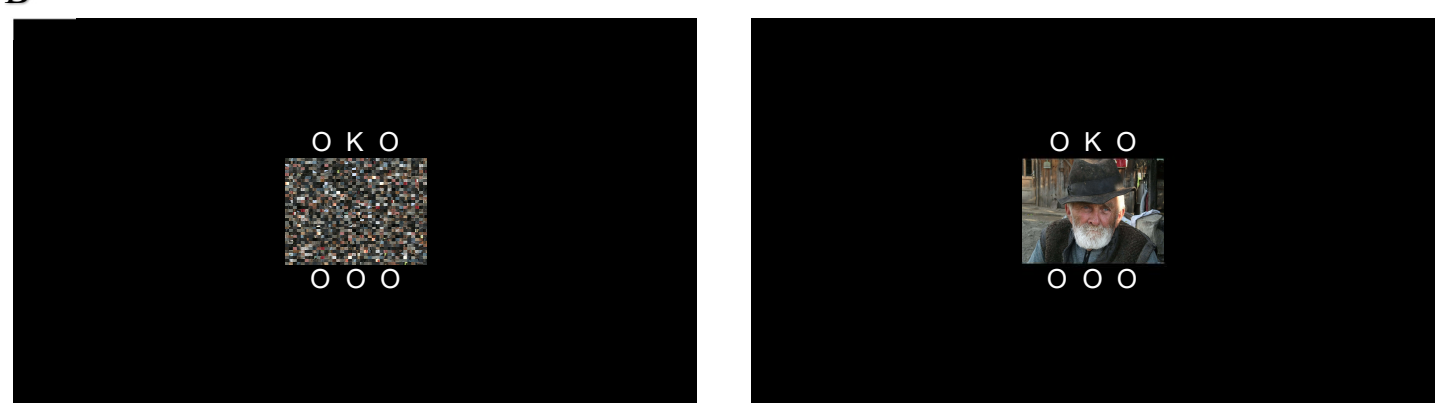

Figure 5. A) Trial structure of Experiment 2 showing a neutral intact-distractor trial B) Examples of a scrambled-distractor trial (left) and a neutral intact-distractor trial (right)

\section{Procedure}

The apparatus used in Experiment 2 was identical to that used in Experiment 1. As shown in Figure 5A, trials were structured identically to the first experiment except that the task array was now presented for $200 \mathrm{~ms}$. On intact-distractor trials, an intact image was presented in the centre of the screen, flanked at the top and bottom by the letter array. On scrambled-distractor trials a pixel-scrambled image was instead presented in the same location.

Participants were assigned to one of three conditions. In the predictable low-frequency condition intact distractors were presented on every fourth trial of a block $(25 \%$ of trials overall). In the unpredictable low-frequency condition intact distractors were present on an unordered $25 \%$ of trials. Lastly, in the unpredictable high frequency condition, intact distractors were present on an unordered $75 \%$ of trials.

The experiment again ran for four blocks of 48 trials, separated by 30 second minimum breaks. Distractor valence was blocked with the block order counterbalanced identically to the previous experiment. After experiment instructions, an additional training block of five trials containing only scrambled images was now included. Following this, participants underwent two training blocks which were representative of the distractor frequency (high or low), and order (predictably every fourth trial or unordered) of the participant's assigned condition.

Participants were informed that all trials would contain an image, and that the image was either intact or scrambled. All participants were also truthfully told what percentage of 
trials would contain an intact distractor and what percentage would not. They were also informed that "an intact image would occur on every fourth trial" (in the predictable lowfrequency condition) or that the order of presentation of scrambled and intact images would be random (in the unpredictable high-frequency and unpredictable low-frequency conditions).

Following the experiment, participants were given a questionnaire (see Appendix B) asking them 1) what the frequency of intact images were, 2) whether it felt as if the images were presented in order, 3) whether they were able to predict upcoming intact distractors and 4) whether they used some strategy to prepare for intact distractor images. If they answered yes to the fourth question, they were also asked to detail what that strategy was. The questionnaire was accompanied by verbal instructions and the chance to ask for clarification.

\section{Design}

This experiment employed a mixed design with Predictability condition (Predictable low-frequency or Unpredictable low-frequency or Unpredictable high-frequency) manipulated between subjects, and Distractor type (Intact or Scrambled) and Valence (Negative or Neutral) manipulated within subjects. The dependent variables were RT (ms), accuracy (\% correct responses to the letter task) and a distraction index (RT(distractor-present) - RT(distractorabsent)). RT data were averaged to the participant level before calculating the distraction index and were then averaged to the group level from where the means and standard deviations shown in Table 2 were derived.

\section{Statistical Analysis}

Preregistration for the present analysis can be found at (https://osf.io/s $8 \mathrm{jtr}$ ) and is provided in Appendix E. Additional analyses are labelled as exploratory. All analyses were conducted only on trials wherein participants responded correctly, and RT was greater than 200 ms. This led to the exclusion of $10.21 \%$ of trials. Two preregistered exclusion criteria were established, excluding participants with lower than 70\% accuracy on blocks of either valence, or accuracy lower than $75 \%$ overall. Two participants met these criteria and were removed from the analysis. Greenhouse Geisser corrections were performed where necessary. Effect sizes were calculated for all inferential statistics. Effect sizes were presented in partial eta squared $\left(\eta_{p}{ }^{2}\right)$ for main effects and interactions. Cohen's $d_{z}$ was used for within subject comparisons (Lakens, 2013).

\section{Questionnaire Items}

For the item "How often were intact images present?" responses were coded as correct (either $25 \%$ or $75 \%$ dependent on predictability condition) or incorrect. The question answers were tallied, and percentages calculated for each response. The number of responses to 
Question 5 (on strategy) were tallied and grouped by similarity of strategy into three categories: responses indicating preferential attention to the task relevant stimuli (e.g. "Checked the top row first, bottom row was covered by doing that"), responses indicating preferential attention to the distractors (e.g. "Squinted my eyes a bit more to avoid having to look at the full intact image") and responses indicating active preparation for the upcoming trial (e.g. "When I knew an intact image was coming, I would look at the letters more") (see Appendix D).

\section{Results}

Analyses were preregistered unless indicated as exploratory. Preregistration is available at (https://osf.io/s8jtr) and is provided in Appendix E.

\section{RT}

Mean RTs and distraction indices by Predictability condition, Valence and Distractor presence are shown in Table 3. Paired samples t-tests indicated significant difference between scrambled-distractor and intact-distractor trials in all conditions excluding neutral distractor blocks in the unpredictable high-frequency condition. Emotional distractors therefore effectively captured attention in all conditions including the unpredictable high-frequency condition whereas neutral distractors were not more distracting than scrambles when distractors occurred frequently.

RTs (shown in Figure 5) were again entered into a 2 (Distractor type: Intact, Scrambled) $\times 2$ (Valence: Neutral, Negative) $\times 3$ (Predictability condition: Predictable low-frequency, Unpredictable low-frequency, Unpredictable high-frequency) mixed ANOVA. As in Experiment 1, no main effect of condition was observed, $F(1,91)=0.46, p=.633, \eta_{p}^{2}=.01$. Significant main effects of valence, $F(1,91)=34.14, p<.001, \eta_{p}{ }^{2}=.27$, and distractor type, $F(1,91)=63.57, p<.001, \eta_{p}{ }^{2}=.41$, were qualified by a significant Valence $\times$ Distractor type interaction, $F(1,91)=27.27, p<.001, \eta_{p}{ }^{2}=.23$. Follow-up paired samples t-tests were conducted, comparing RT on intact-distractor trials to scrambled-distractor trials (i.e. distraction) in neutral and negative distractor blocks. Both on negative blocks, $t(93)=7.37, p$ $<.001, d_{z}=0.76$, and neutral blocks, $t(93)=3.98, p<.001, d_{z}=0.41$, RTs were greater when distractors were intact. However, this difference was far greater in the negative $(M=52, S D=$ $69)$ than in the neutral trial blocks $(M=14, S D=35)$. Participants were therefore substantially more distracted in the negative as opposed to the neutral blocks across predictability. As in Experiment 1, the emotional irrelevant-distractor paradigm was able to effectively elicit emotional distraction.

A critical test of the experiments' hypotheses centres on the Predictability condition $x$ Distractor type interaction, which was again found to be significant, $F(2,91)=4.71, p=.011$, 
$\eta_{p}^{2}=.09$. Follow-up t-tests indicate that RT was greater in response to intact-distractor trials in the predictable low-frequency, $t(31)=4.96, p<.001, d_{z}=0.88$, unpredictable low-frequency, $t(30)=5.41, p<.001, d_{z}=0.97$, and unpredictable high-frequency, $t(30)=3.79, p<.001, d_{z}=$ 0.68 , conditions. The difference between intact-distractor and scrambled-distractor trials was greatest in the predictable low-frequency condition $(M=47, S D=74)$, followed by the unpredictable low-frequency condition $(M=36, S D=51)$ and then the unpredictable highfrequency condition $(M=16, S D=37)$. Thus, as in the first experiment, distraction was found in all conditions but was found to be greatest in the predictable low-frequency condition. To further assess the magnitude of this difference and determine whether this interaction reflected a paradoxical expectation effect, distraction indices (see Figure 7) were entered into a 2 (Valence: Neutral, Negative) × 3 (Predictability condition: Predictable low-frequency, Unpredictable low-frequency, Unpredictable high-frequency) mixed ANOVA. Analysis revealed a main effect of predictability condition, $F(2,91)=4.71, p=.011, \eta_{p}{ }^{2}=.09$. A Tukey's honest significant difference test found that distraction in the predictable low-frequency and unpredictable high-frequency conditions differed significantly, $p=.009$. However, no significant difference was observed between the unpredictable high and low-frequency groups, $p=.127$, nor between the unpredictable low-frequency and predictable low-frequency groups, $p=.551$. 
Table 3

Mean (SD) RTs (in ms), Distraction indices and paired samples $t$-test results, including confidence intervals and effect sizes (Cohen's $d_{z}$ ), by Valence, Predictability condition and Distractor type in Experiment 2

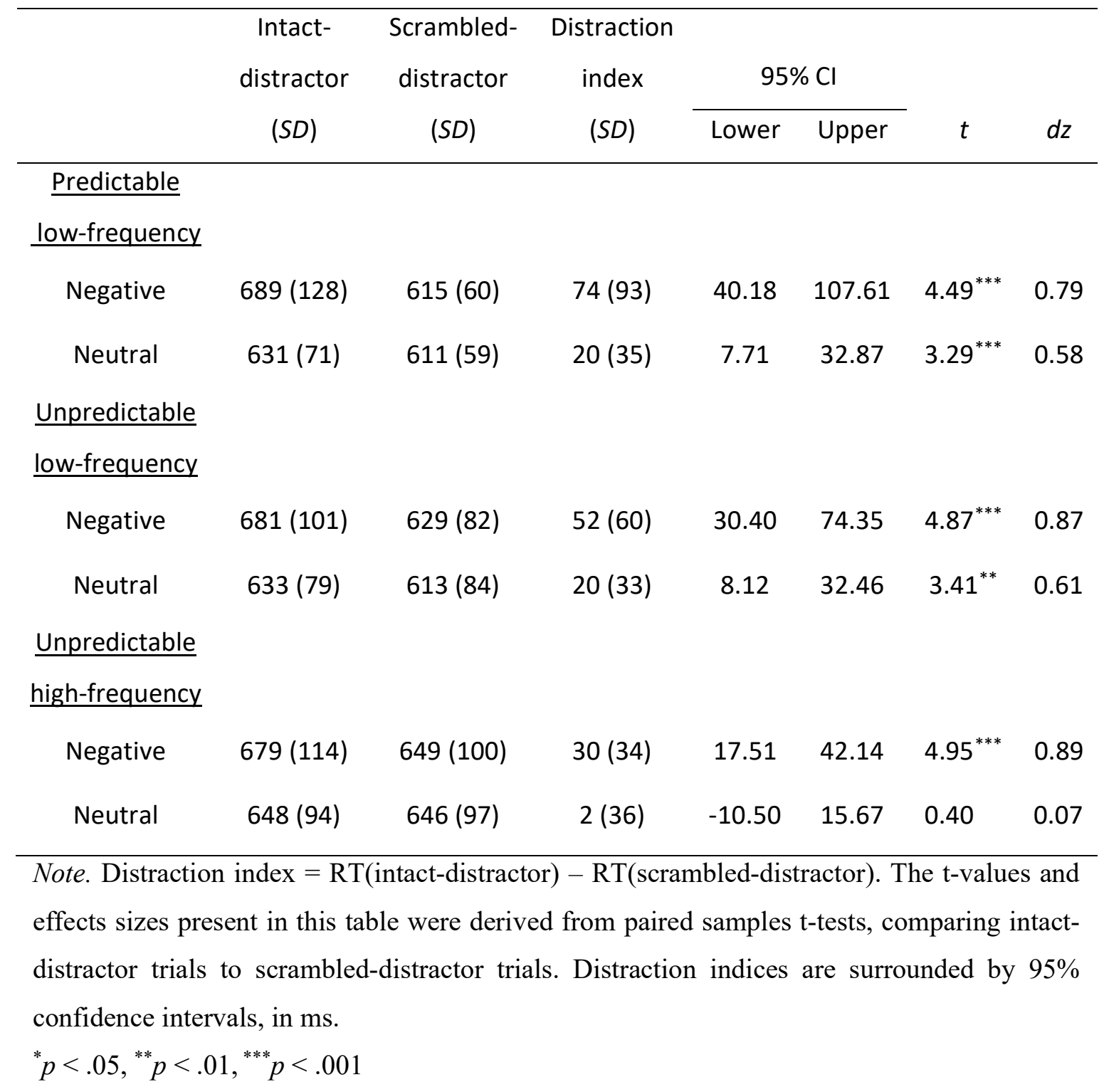




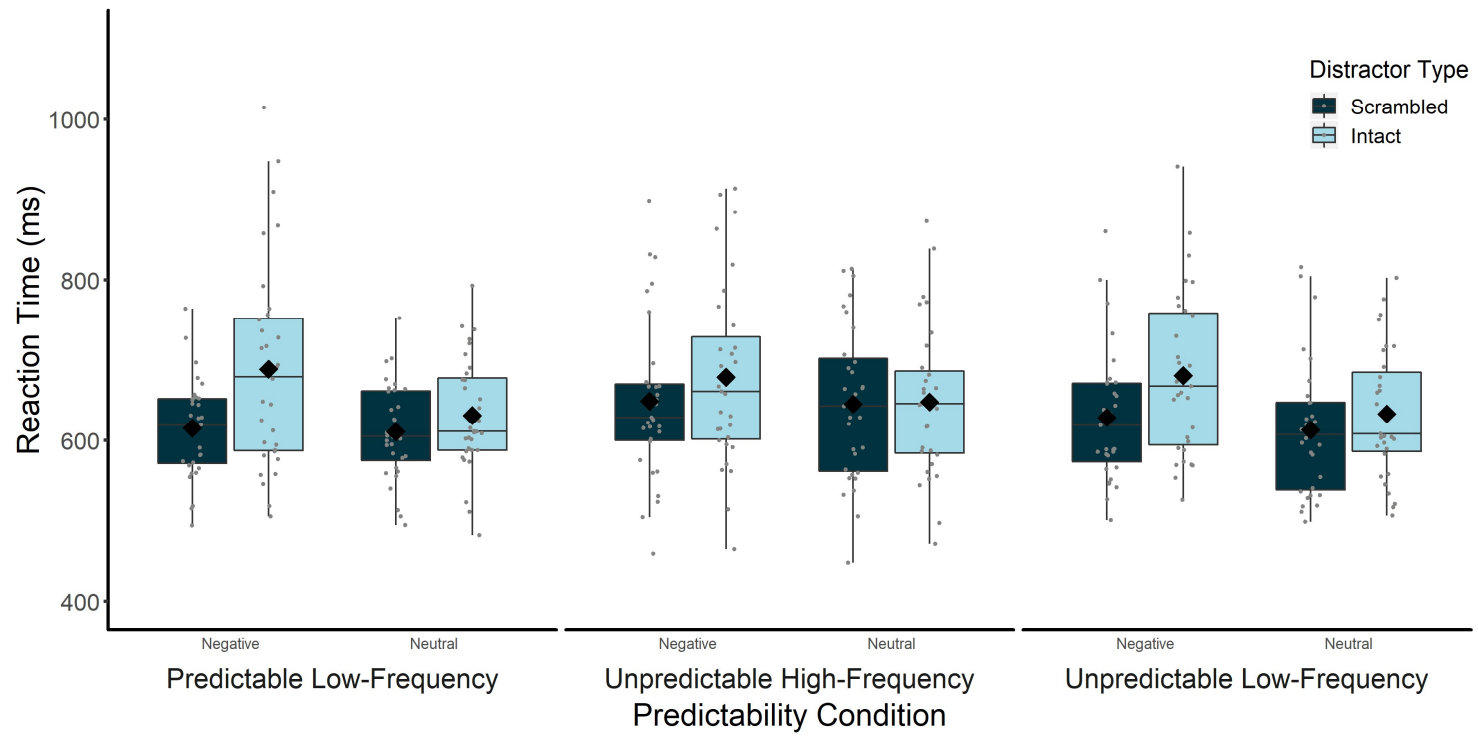

Figure 6. Experiment 2 individual RTs, by Predictability condition, Valence and Distractor type

Note. Diamonds indicate mean RT. Boxes contain the middle quartiles and the bar indicates median RT.

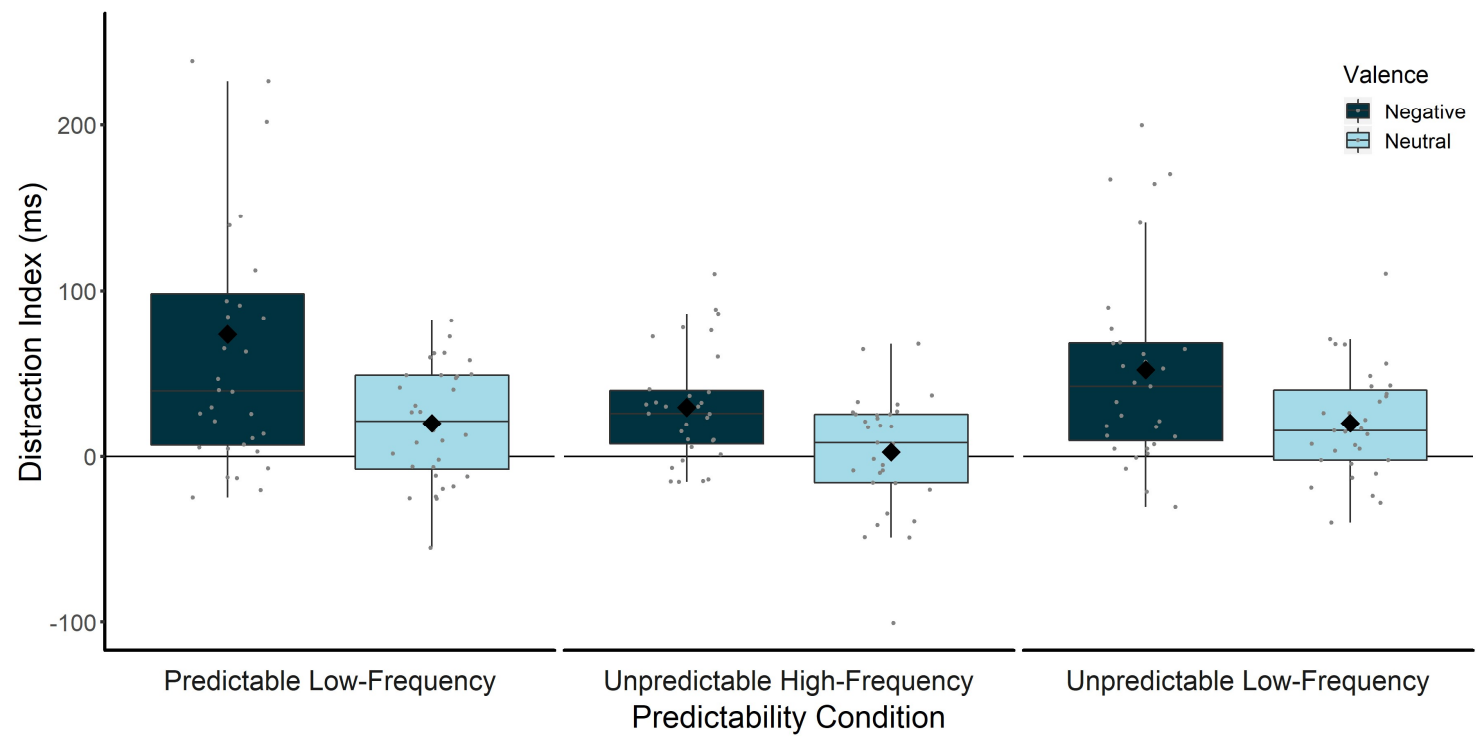

Figure 7. Experiment 2 individual Distraction indices, by Predictability condition, Valence and Distractor type

Note. Diamonds indicate mean Distraction index. Boxes contain the middle quartiles and the bar indicates median Distraction index.

\section{Exploratory Frequency Manipulation Replication}

The unpredictable high-frequency condition was included in Experiment 2 to replicate the typical distractor frequency effect. As frequency effects have been shown consistently in 
the literature, an explicit replication of the frequency effect of emotional distraction was also attempted, comparing distraction indices only between the unpredictable low-frequency and high-frequency conditions ${ }^{7}$. An exploratory 2 (Valence: Neutral, Negative) $\times 2$ (Distractor frequency: Low, High) mixed ANOVA was conducted. Results of this analysis indicated a main effect of both frequency, $F(1,60)=6.38, p=.014, \eta_{p}{ }^{2}=.10$, and Valence, $F(1,60)=$ $17.29, p<.001, \eta_{p}{ }^{2}=.22$, reflecting greater distraction indices when distractor frequency was low $(M=36, S D=50)$ relative to high $(M=16, S D=37)$ and when distractors were negative $(M=41, S D=49)$ as opposed to neutral $(M=11, S D=35)$. Thus, the current experiment replicates the expected distractor frequency effect. However, no Frequency $\times$ Valence interaction was found, $F(1,60)=0.12, p=.735, \eta_{p}{ }^{2}=.002$, meaning that, while distraction overall was lower in the high frequency condition, emotional distraction was not.

These results provide evidence of lower distraction when distractor frequency is increased, supporting the standpoint that the unpredictable low-frequency and unpredictable high-frequency conditions in the present experiment elicit behavioural performance in line with reactive and proactive control mechanisms respectively (Grimshaw et al., 2018; Schmidts et al., 2020; Walsh et al., 2018, 2019).

\section{Accuracy}

Accuracy scores (Figure 8 and Table 4) were lower in Experiment 2 overall with participants committing an average of $15.12(S D=8.89)$ errors, equivalent to an average of $7.87 \%$ of trials. To address the concerns of a speed-accuracy trade off, and to clarify the effect of expectation on accuracy, accuracy scores were entered into a 2 (Distractor type: Intact, Scrambled) $\times 2$ (Valence: Neutral, Negative) $\times 3$ (Predictability condition: Predictable lowfrequency, Unpredictable low-frequency, Unpredictable high-frequency) mixed ANOVA. Unlike Experiment 1, no main effect of condition was observed. A main effect of Distractor type, $F(1,91)=11.65, p<.001, \eta_{p}^{2}=.11$, was qualified by a Predictability condition $\times$ Distractor type interaction, $F(1,91)=3.66, p=.030, \eta_{p}{ }^{2}=.07$. Contrary to what would be expected given a speed-accuracy trade off, follow-up paired samples t-tests indicated higher accuracy on scrambled-distractor trials $(M=93.7, S D=4.8)$ than on intact-distractor trials $(M$ $=89.9, S D=9.4)$ in the predictable low-frequency condition, $t(63)=3.45, p=.001, d_{z}=0.43$, but not in the unpredictable high-frequency nor the unpredictable low-frequency conditions $p$ $>.05$. There was no interaction with valence.

\footnotetext{
${ }^{7}$ Not including the predictable low-frequency condition.
} 


\section{Table 4}

Mean (SD) accuracy scores (in \% correct trials) and paired samples t-test results, including confidence intervals and effect sizes (Cohen's $d_{z}$ ), by Valence, Predictability Condition and

Distractor type in Experiment 2

\begin{tabular}{|c|c|c|c|c|c|c|c|}
\hline & \multirow{2}{*}{$\begin{array}{l}\text { Intact- } \\
\text { distractor } \\
(S D)\end{array}$} & \multirow{2}{*}{$\begin{array}{l}\text { Scrambled- } \\
\text { distractor } \\
(S D)\end{array}$} & \multirow{2}{*}{$\begin{array}{l}\text { Accuracy } \\
\text { difference } \\
(S D)\end{array}$} & \multicolumn{2}{|c|}{$95 \% \mathrm{Cl}$} & \multirow[b]{2}{*}{$t$} & \multirow[b]{2}{*}{$d z$} \\
\hline & & & & Lower & Upper & & \\
\hline \multicolumn{8}{|c|}{$\begin{array}{c}\text { Predictable } \\
\text { low-frequency }\end{array}$} \\
\hline Negative & $88.80(10.97)$ & 93.79 (4.96) & 4.99 (10.42) & -8.75 & -1.24 & $2.71^{*}$ & 0.48 \\
\hline \multicolumn{7}{|c|}{$\underline{\text { Unpredictable }}$} & 0.38 \\
\hline \multicolumn{8}{|c|}{ low-frequency } \\
\hline Negative & $90.32(7.71)$ & $92.88(5.14)$ & $2.56(7.38)$ & -5.26 & 0.15 & 1.93 & 0.35 \\
\hline \multicolumn{8}{|c|}{$\underline{\text { Unpredictable }}$} \\
\hline high-freque & & & & & & & \\
\hline Negative & $90.68(6.11)$ & 90.86 (7.79) & $0.18(6.22)$ & -2.46 & 2.10 & 0.16 & 0.03 \\
\hline Neutral & 91.80 (5.09) & $92.34(7.69)$ & $0.54(7.16)$ & -3.16 & 2.09 & 0.42 & 0.08 \\
\hline
\end{tabular}

Note. The t-values and effects sizes present in this table are taken from paired samples t-tests, comparing scrambled-distractor to intact-distractor trials. Accuracy differences are surrounded by $95 \%$ confidence intervals.

${ }^{*} p<.05,{ }^{* *} p<.01,{ }^{* * *} p<.001$ 


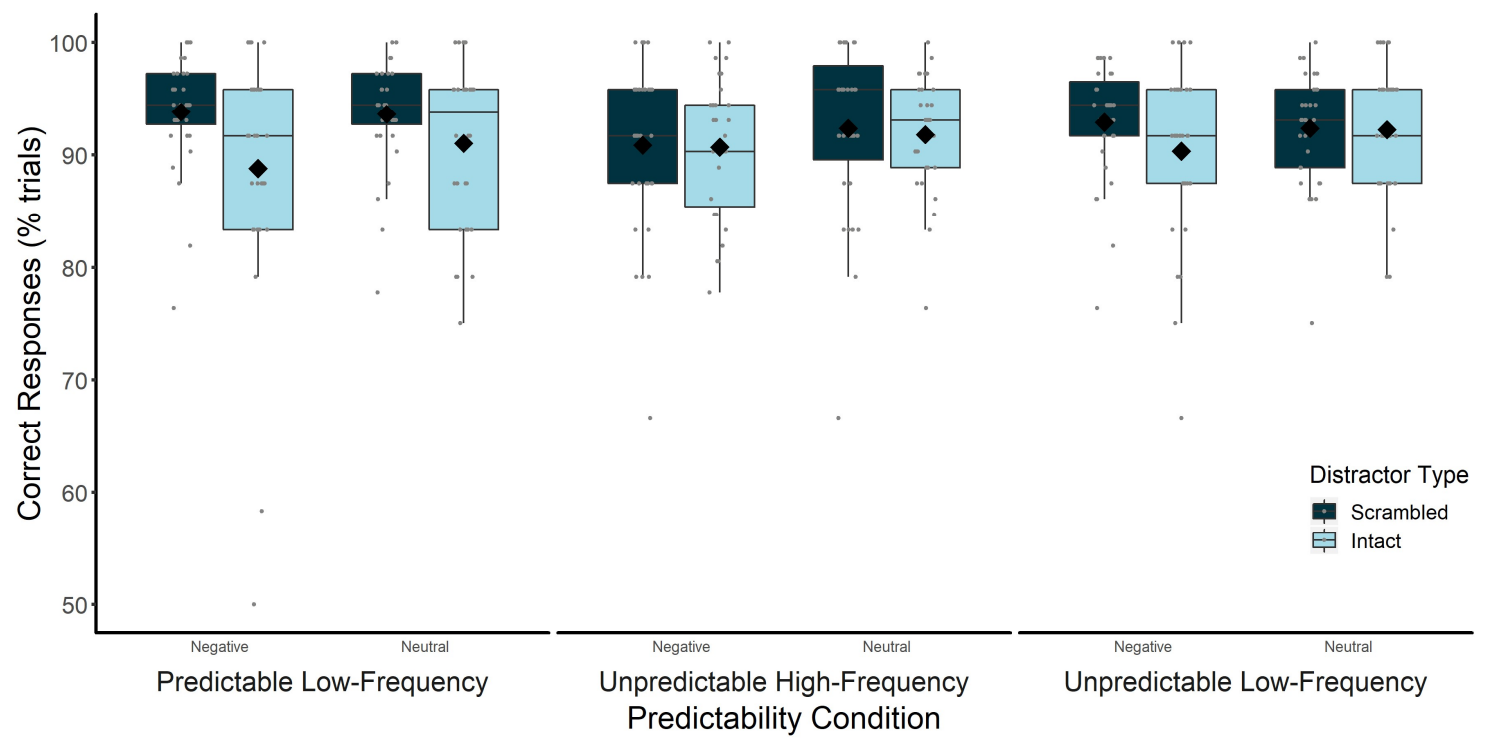

Figure 8. Experiment 2 individual Accuracy scores by Predictability condition, Valence and Distractor type

Note. Diamonds indicate mean Accuracy score. Boxes contain the middle quartiles and the bar indicates median Accuracy score.

\section{Questionnaire Responses}

Responses to the post-experiment questionnaire may provide some insight into participants' subjective experience of the task. An exploratory Pearson's chi-square test was used to examine whether the responses to the individual questions differed between groups.

\section{Question 1}

Tallies of participant answers to the post-experiment questionnaire are shown in Table 5. The first question asked, "How often were intact images present?". Across conditions, most participants $(82 \%)$ responded correctly when asked what percentage of trials contained an intact distractor image. Participants in the predictable low-frequency condition responded correctly most often (91\%) followed by the unpredictable low-frequency condition (88\%) and then the unpredictable high-frequency condition (69\%). Nevertheless, many participants, particularly those in the unpredictable high-frequency condition, answered incorrectly although the distractor frequency was explicitly stated in the experiment instructions. A chi-square test of goodness of fit indicated that correct responses to the first question were not equally distributed, $X^{2}(2, N=96)=6.15, p=.046$, reflecting more incorrect responses specifically in the unpredictable high-frequency condition.

\section{Question 2}


The second question asked, "Did you feel as if the images were presented to you in a set order?". It was expected that participants would answer "Yes" for the predictable lowfrequency condition and "No" in the unpredictable conditions (given the transparency of instructions). Many participants (72\%) in the predictable low-frequency condition reported that distractors were presented in a set order. A substantial portion of participants also indicated that distractors were presented in a set order in the unpredictable high-frequency condition $(31 \%)$. Few (6\%) reported this in the unpredictable low-frequency condition. As with the first question, a chi-square test of goodness of fit indicated that correct responses to the second question were not equally distributed, $X^{2}(2, N=96)=30.31, p<.001$, suggesting that participants in the predictable low-frequency condition were reasonably aware of the predictable order of distractors.

\section{Question 3}

The third question asked, "Were you able to predict when an intact image would be present on the following trial?". Participants in the unpredictable conditions responded as would be expected, with few stating they could predict any upcoming distractors (Highfrequency $=9 \%$, Low-frequency $=6 \%$ ). Substantially more participants reported that they were able to predict distractors in the predictable low-frequency condition (69\%). A chi-square test of goodness of fit indicated that responses to Question 3 were not equally distributed, $X^{2}(2, N$ $=96)=39.27, p<.001$.

This result raises the question of whether there is any difference between the individuals in the predictable low-frequency condition who felt as if they could predict distractors $(n=23)$ and those who felt they could not $(n=9)$. It is possible that the individuals who report that they could predict the distractors were consciously making use of the pre-trial period to prepare for upcoming distractors. To test whether distraction differed based on the subjective feeling of predictability an exploratory 2 (Valence: Neutral, Negative) $\times 2$ (Subjective prediction: Could Predict, Could Not Predict) mixed ANOVA was conducted on distraction indices.

Only a main effect of valence was found, $F(1,30)=8.18, p=.007, \eta_{p}{ }^{2}=.21$, reflecting greater distraction by negative $(M=74, S D=93)$ as opposed to neutral distractors $(M=20, S D$ $=35$ ). Hence, no substantial difference was found between participants in the predictable lowfrequency condition based on their subjective experience of predictability. In fact, participants who reported they were able to predict upcoming distractors were, numerically albeit not statistically, more distracted $(M=51, S D=76)$ than those who did not $(M=39, S D=72)$ again possibly alluding to a harmful and valence specific effect of expectation on distraction.

\section{Question 4}


The fourth question asked, "Did you use any strategy to prepare for the intact images on upcoming trials?". Participants in the unpredictable high-frequency condition indicated the use of a strategy least often (9\%) with more "Yes" answers by the unpredictable low-frequency condition (22\%) and the most in the predictable low-frequency condition (28\%). A chi-square test of goodness of fit indicated that responses to the fourth question were equally distributed, $X^{2}(2, N=96)=3.68, p=.159$. Thus, participants across the conditions were equally likely to use a strategy to engage with intact-distractor trials.

Table 5

Tallies and percentages (\%) of responses to post-experiment questionnaire items across conditions

\begin{tabular}{|c|c|c|c|c|}
\hline & $\begin{array}{c}\text { Predictable } \\
\text { low-frequency }\end{array}$ & $\begin{array}{l}\text { Unpredictable } \\
\text { high-frequency }\end{array}$ & $\begin{array}{l}\text { Unpredictable } \\
\text { low-frequency }\end{array}$ & Total \\
\hline \multicolumn{5}{|l|}{ Question 1} \\
\hline Correct & $29(90.63)$ & $22(68.75)$ & $28(87.50)$ & 79 (82.29) \\
\hline Incorrect & $3(9.38)$ & $10(31.25)$ & $4(12.50)$ & $17(17.71)$ \\
\hline \multicolumn{5}{|l|}{ Question 2} \\
\hline Yes & $23(71.88)$ & $10(31.25)$ & $2(6.25)$ & $35(36.46)$ \\
\hline No & $9(28.13)$ & $22(68.75)$ & 30 (93.75) & $61(63.54)$ \\
\hline \multicolumn{5}{|l|}{ Question 3} \\
\hline Yes & $22(68.75)$ & $3(9.38)$ & $2(6.25)$ & $27(28.13)$ \\
\hline No & $10(31.25)$ & $29(90.63)$ & $30(93.75)$ & $69(71.88)$ \\
\hline \multicolumn{5}{|l|}{ Question 4} \\
\hline Yes & $9(28.13)$ & $3(9.38)$ & $7(21.87)$ & 19 (19.79) \\
\hline No & $23(71.87)$ & 29 (90.63) & 25 (78.13) & $77(80.21)$ \\
\hline
\end{tabular}

\section{Question 5}

The final question asked participants to detail what strategies they used if they indicated that they had used a strategy to prepare for upcoming intact-distractor trials. The 18 responses to Question 5 were separated into three categories: responses indicating preferential attention to the task relevant stimuli, responses indicating preferential attention to the distractors and responses indicating active preparation in advance of the upcoming trial. All responses are listed in Appendix D with counts of responses for each category displayed in Table 6. 
Of those who responded, participants did not often preferentially attend to task relevant stimuli as a way of enhancing task performance or avoiding distraction. Most (13 responses) concerned described a strategy detailing avoidance of the distractors. Whatever deliberate effort participants apply to control attention, at least subjectively, appears to be directed at avoiding the distractors themselves. Alternatively, when pressed for a strategy, participants may have referenced the distractors as they were salient in comparison to the dull and repetitive task. Only three participants (all from the predictable low-frequency condition), provided a response which indicated advance preparation for upcoming intact-distractor trials. Statistical analysis of how these three differed from the rest of the predictable low-frequency condition was not attempted given the low number of participants. However, based on these findings, one can speculate that at least some participants in the predictable low-frequency condition may be making strategic use of the foreknowledge of distractors.

Table 6

Tallied responses to question 5 of the post-experiment questionnaires, separated by response similarity and Predictability condition

\begin{tabular}{ccccc}
\hline & $\begin{array}{c}\text { Predictable } \\
\text { low-frequency }\end{array}$ & $\begin{array}{c}\text { Unpredictable } \\
\text { high-frequency }\end{array}$ & $\begin{array}{c}\text { Unpredictable } \\
\text { low-frequency }\end{array}$ & Total \\
\hline $\begin{array}{c}\text { Response regarding } \\
\text { distractors }\end{array}$ & 4 & 2 & 7 & 13 \\
Response regarding \\
the task relevant \\
stimuli
\end{tabular}




\section{Conflict Adaptation}

Both Experiment 1 and 2 suggests that expectation alone does not improve the ability to avoid emotional distractors. Experiment 2 also indicates that increased distractor frequency leads to greater control over attention. Hence, proactive control has been shown to be driven not by expectation but by experience of previous distraction. It should be noted that proactive control is not a singular mechanism but rather a collection of mechanisms which act similarly. Thus, several mechanisms could account for how control is altered proactively via experience. A conflict adaptation standpoint would argue that cognitive control is increased in the short term after conflict exposure as a form of dynamic proactive control. Many have previously shown a decrease in the effect of conflict on trials following conflict-present trials relative to non-conflict trials (Botvinick et al., 2001). Conflict adaptation has been observed in response conflict tasks such as Stroop (Purmann \& Pollmann, 2015) and flanker paradigms (Ullsperger et al., 2005). In studies manipulating distractor frequency, the low-frequency condition will have few trials where an intact-distractor trial follows another, simply due to the rarity of these trials. Conversely, the high-frequency condition will have more of these consecutive intactdistractor trials simply by chance, due to the increased frequency of intact-distractor trials. Hence, increased cognitive control due to increased distractor frequency may be due not to increased expectation of upcoming distractors, but rather, to increased incidence of conflict adaptation. In the predictable low-frequency condition, there were no consecutive intactdistractor trials, as trials followed a scrambled, scrambled, scrambled, intact pattern. This could explain why the distraction indices in the predictable low-frequency condition more closely resembled those the unpredictable low-frequency condition than the unpredictable highfrequency condition. Possibly, the paradoxical expectation effect could also be explained in that sequential intact-distractor trials still occur in the unpredictable low-frequency condition (albeit infrequently), reducing distraction relative to the predictable low frequency condition.

To assess the effect of conflict adaptation on distraction, the RTs to trials preceded by a scrambled-distractor trial (a non-conflict trial) were compared to those preceded by an intactdistractor trial (a conflict trial) in the unpredictable low and high-frequency conditions (see Figure 9). A 2 (Valence: Neutral, Negative) $\times 2$ (Previous trial: Conflict, Non-conflict) $\times 2$ (Frequency: High, Low) $\times 2$ (Distractor type: Intact, Scrambled) mixed ANOVA was conducted comparing just the unpredictable low and high-frequency conditions. The effect of conflict adaptation on distraction would be indicated by an interaction between Previous trial type, Frequency and Distractor type reflecting lower RTs when intact-distractor as opposed to scrambled-distractor trials follow intact-distractor trials, specifically in the unpredictable high- 
frequency condition. Results indicated a main effect of Distractor type, $F(1,60)=34.85, p<$ $.001, \eta_{p}{ }^{2}=0.37$, which was qualified by a Distractor type $\times$ Frequency interaction, $F(1,60)=$ $7.23, p=.009, \eta_{p}^{2}=0.11$. Since Distractor type did not interact with Previous trial these effects were not further explored ${ }^{8}$.

A main effect of Valence, $F(1,60)=11.84, p=.001, \eta_{p}{ }^{2}=0.17$, was however qualified by an interaction effect between Valence and Distractor type, $F(1,60)=16.39, p<.001, \eta_{p}{ }^{2}=$ 0.22 , and a three way Frequency $\times$ Valence $\times$ Previous trial interaction, $F(1,60)=4.83, p=$ $.032, \eta_{p}{ }^{2}=0.07$. To assess whether the three way interaction reflects slower responding in the high frequency condition (in either the neutral or negative trials) when the previous trial contained an intact as opposed to scrambled distractor, RT scores were split by Frequency $($ High $\times$ Low $)($ Figure 9) and were entered into a follow-up 2 (Valence: Neutral, Negative) $\times 2$ (Previous trial: Conflict, Non-conflict) mixed ANOVA.

Considering only low-frequency trials, only a main effect of Valence was observed, $F(1,30)=16.59, p<.001, \eta_{p}{ }^{2}=0.36$, indicating greater RT in blocks containing negative $(M$ $=656, S D=106)$ over blocks containing neutral $(M=627, S D=101)$ distractors. In the highfrequency condition, only the Valence $\times$ Previous trial interaction was significant, $F(1,30)=$ $4.60, p=.040, \eta_{p}{ }^{2}=0.13$. Additional follow-up t-tests indicated that only in the unpredictable high-frequency condition, when an intact distractor was present on the previous trial, were participants slower to respond to trials containing negative $(M=665, S D=106)$ as opposed to neutral distractors $(M=642, S D=91), t(30)=3.87, p<.001, d_{z}=0.70$.

There was therefore some evidence that conflict adaptation modified RTs. However, there was no interaction with Distractor type. Therefore, there is no evidence that conflict on a previous trial alters distraction. Hence there is no support for trial-by-trial dynamic upregulation of control as the method by which experience modifies control in the unpredictable high-frequency condition.

\footnotetext{
${ }^{8}$ See instead the exploratory frequency manipulation replication to illustrate differences in distraction between the unpredictable low and high frequency groups.
} 


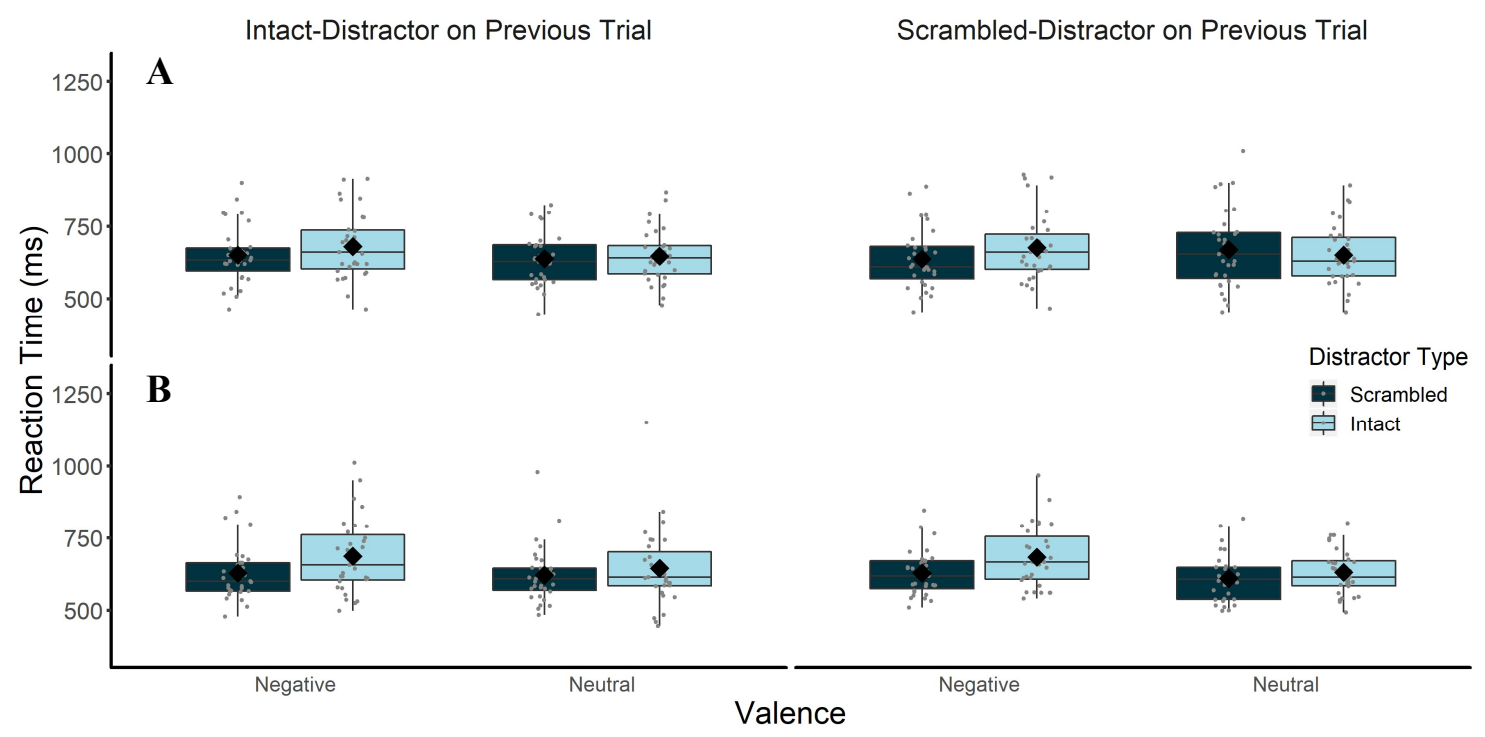

Figure 9. A) Individual RTs by Previous trial type, Valence and Distractor type for trials in the unpredictable high-frequency condition of Experiment 2 B) Individual RTs by Previous trial type, Valence and Distractor type for trials in the unpredictable low-frequency condition in Experiment

Note. Diamonds indicate mean RT. Boxes contain the middle quartiles and the bar indicates median RT.

The above analysis cannot include the predictable low-frequency condition due to there being no sequential intact-distractor trials, by design. However, if participants exert greater cognitive control after exposure to a distractor, this might be reflected in subsequent RTs to scrambled-distractor trials. As trials progress in sequence, it would further be expected that the benefit to control due to conflict adaptation would decrease. Hence scrambled-distractor RTs in the predictable low-frequency condition (Figure 10) were compared using an exploratory 2 (Valence: Neutral, Negative) $\times 2$ (Trials since distractor: 1, 2, 3) repeated measures ANOVA specifically assessing the linear effect of trials since distractor.

Rather than increase, RTs on scrambled-distractor trials decreased with time since an intact distractor: one trial $(622 \mathrm{~ms})$, two trials $(612 \mathrm{~ms})$, and then three trials $(605 \mathrm{~ms})$. The repeated measures ANOVA indicated only a main effect of distance from an intact-distractor trial, $F(2,62)=5.15, p=.010, \eta_{p}{ }^{2}=0.14$. Furthermore, the linear effect (RT decreasing over trials) was found to be significant, $F(1,31)=10.16, p=.002, \eta_{p}{ }^{2}=0.25$, indicating that participants did not upregulate control specifically on the trial following conflict exposure.

This analysis indicates that there is no evidence across any of the groups in the present experiment for conflict adaptation on trials following an intact-distractor trial as a method of inducing experience-based control. It should be noted however, that due to the design of the 
experiment, few trials were available for analysis, particularly in the unpredictable highfrequency condition. Hence, conclusions drawn from these analyses are speculative.

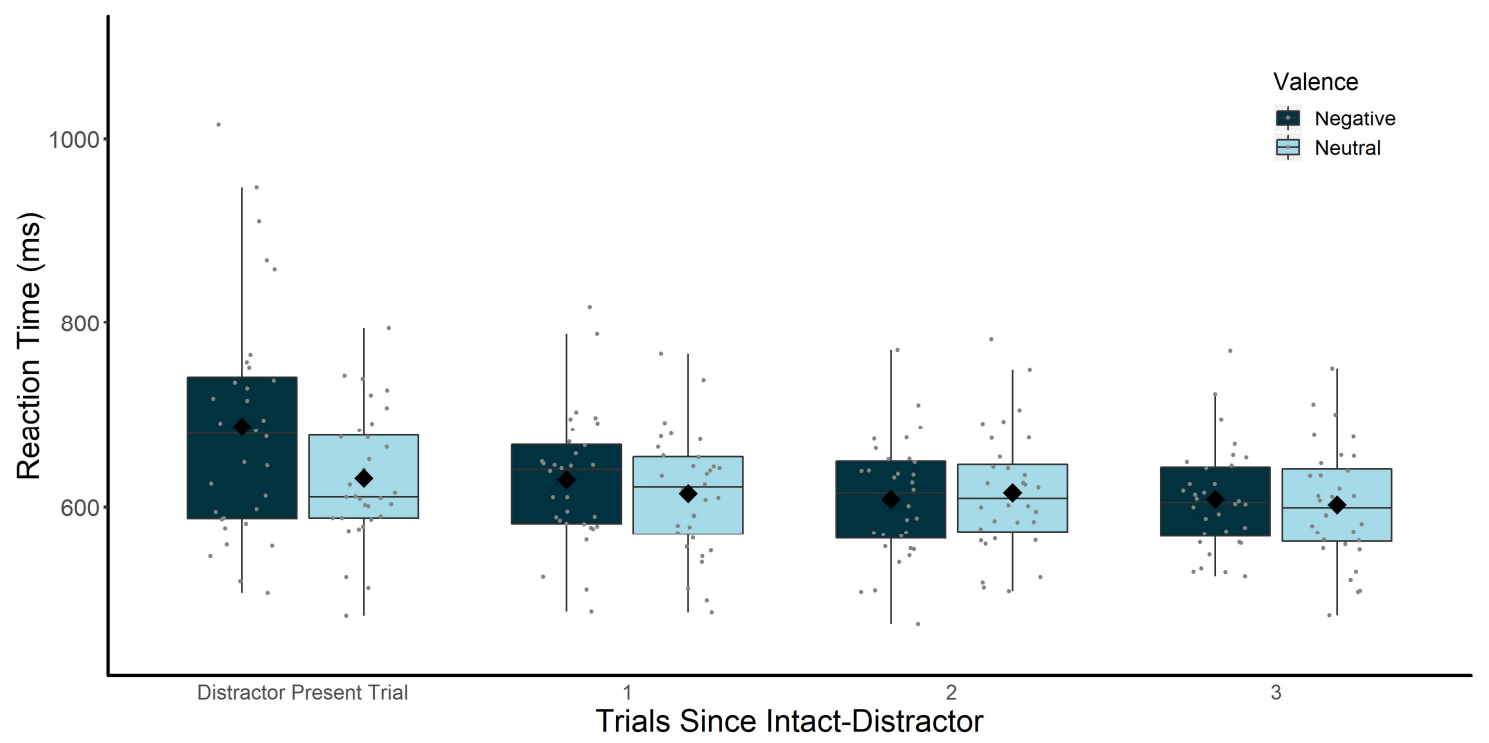

Figure 10. Experiment 2 scrambled-distractor trial RTs in the predictable low-frequency condition by Valence and Number of trials since an intact-distractor Note. Diamonds indicate mean RT. Boxes contain the middle quartiles and the bar indicates median RT. Trials wherein an intact distractor is present are included for illustrative purposes (leftmost).

\section{Change in Distraction Over Time}

The results suggest that experience may be a more important factor in biasing control mechanisms than expectation of upcoming distractors. Experience, however, is likely not acquired instantly. It is therefore expected that some exposure to distractors is required for experience to modify cognitive control (as in Wiemers \& Redick, 2018). Hence, to assess whether cognitive control improved as experience was gained, the distraction indices for the first block of each valence of distractor were compared to the second (shown in Figure 11). An exploratory 2 (Valence: Neutral, Negative) $\times 2$ (Block: First, Second) $\times 3$ (Predictability condition: Predictable low-frequency, Unpredictable low-frequency, Unpredictable highfrequency) mixed ANOVA was conducted. Analyses indicated a main effect of Predictability condition $^{9}, F(2,91)=4.66, p=.012, \eta_{p}{ }^{2}=0.09$, and Valence ${ }^{10}, F(1,91)=26.9, p<.001, \eta_{p}{ }^{2}=$

\footnotetext{
${ }^{9}$ A Tukeys HSD showed only, as in the previous distraction index analysis, significantly greater distraction in the predictable $(M=47, S D=82)$ over the unpredictable high frequency condition $(M=16, S D=52), p=.009$. ${ }^{10}$ Indicating increased distraction by negative $(M=52, S D=77)$ over neutral $(M=15, S D=51)$ distractors.
} 
0.23 , without an effect of Block nor any interactions, counter to what would be expected if participants improved over time.

It may also be that not much exposure is needed before control is modified. To further assess whether participants improved with experience acquisition, performance was assessed only within the first block of trials where experience was likely to cause the greatest change. The trials in the first block of the experiment were separated into three groups: the initial third, second third and final third of trials, capturing the start, middle and end of the block. Figure 12 shows the mean RTs for each condition when divided in this way. Mean RTs for each condition trended differently, rising in the predictable low-frequency condition, decreasing in the unpredictable low-frequency condition and remaining steady in the unpredictable highfrequency condition. To clarify the nature of these changes an exploratory 2 (Block progress: Start, Middle, End) $\times 3$ (Predictability condition: Predictable low-frequency, Unpredictable low-frequency, Unpredictable high-frequency) $\times 2$ (Distractor type: Intact, Scrambled) mixed ANOVA was completed, finding only an effect of Distractor type ${ }^{11}, F(1,88)=18.00, p<.001$, $\eta_{p}{ }^{2}=0.17$, providing no evidence that experience of distraction over time reduced distraction.

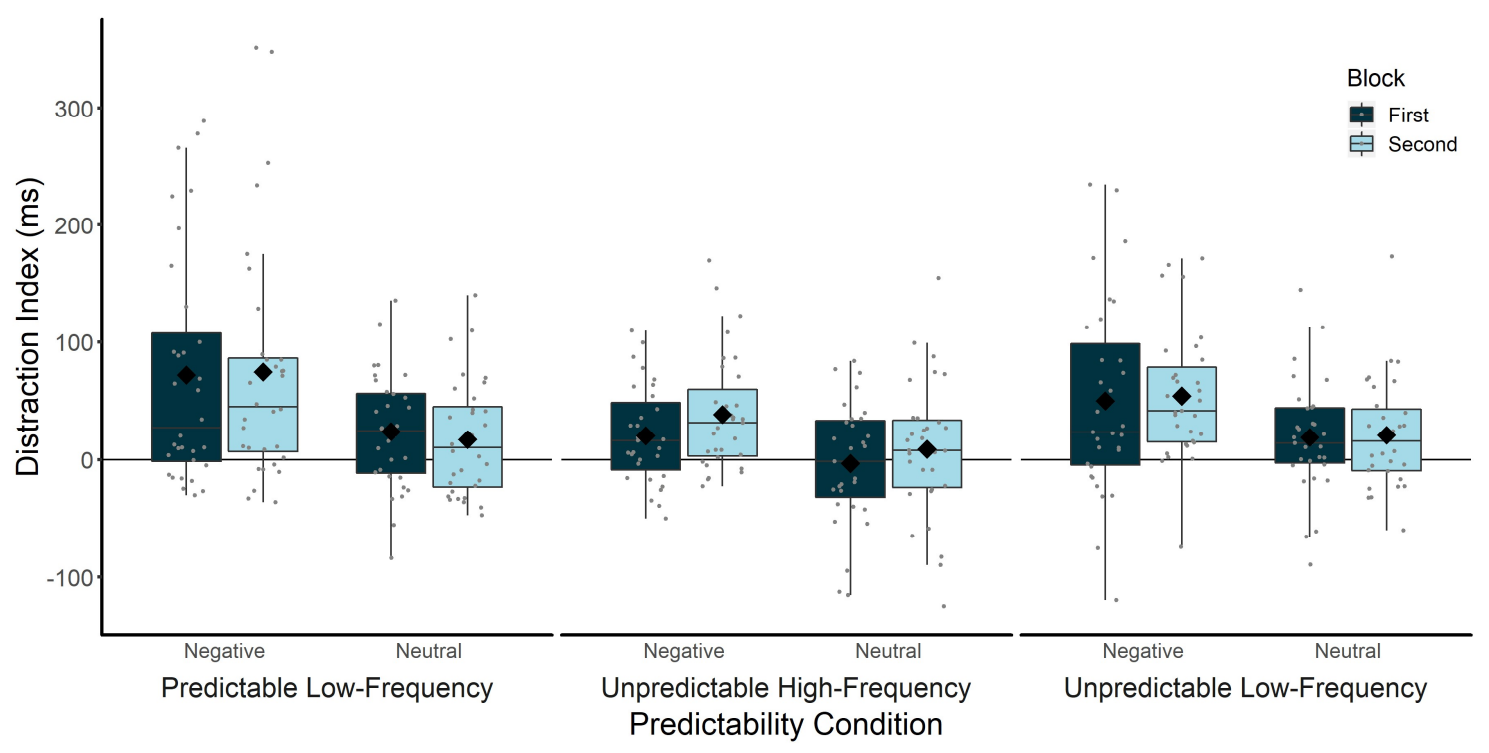

Figure 11. Experiment 2 Distraction indices by Predictability condition, Valence and Block Note. Diamonds indicate mean Distraction index. Boxes contain the middle quartiles and the bar indicates median Distraction index.

\footnotetext{
${ }^{11}$ Indicating greater RTs to intact-distractor $(M=677, S D=131)$ than to scrambled-distractor trials $(M=640$, $S D=109)$
} 


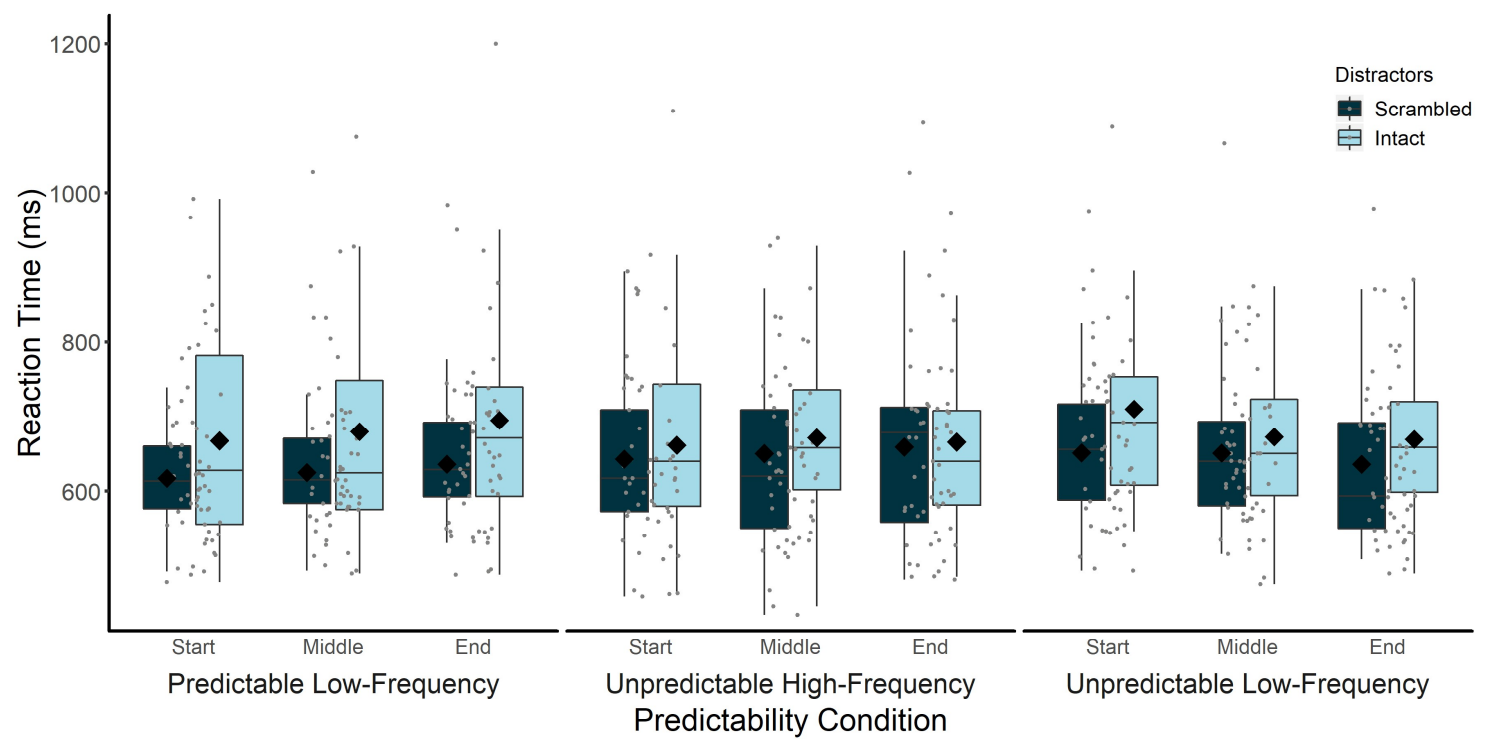

Figure 12. Experiment 2 RTs for the first block of the experiment divided into the first, middle and final 16 trials, by Predictability condition and Distractor type

Note. Diamonds indicate mean RT. Boxes contain the middle quartiles and the bar indicates median RT.

\section{Discussion}

Experiment 2 set out to replicate the unexpected results of Experiment 1 and further test the hypothesis that expectation of upcoming distractors improves control. A high frequency unpredictable distractor condition was also included in the replication to compare the effect of expectation to the effect of distractor frequency. Experiment 2, in a direct test of the effect, replicates previous frequency manipulations (Grimshaw et al., 2018; Schmidts et al., 2020) in that distraction was lower in the unpredictable high-frequency condition than in the unpredictable low-frequency condition. However, emotional distraction was still observed in both the unpredictable high-frequency and unpredictable low-frequency conditions. Hence, while emotional distraction was less prominent when distractors were frequent, the present study did not provide evidence that increased distractor frequency allows participants to control their attention well enough to ignore emotional distractors as they did neutral distractors.

Regarding the effect of expectation, three competing predictions were proposed. The first mirrored the predictions of Experiment 1. In support of expectation-based strategically implemented proactive control distraction was predicted to be greater in the unpredictable lowfrequency condition than in the predictable low-frequency condition. This was contrasted by the second prediction, derived from the unexpected results of the first study, which stated that expectation of upcoming distractors does not necessarily bias control and therefore, distraction 
should not differ between the unpredictable low-frequency condition and the predictable lowfrequency condition. The third alternative prediction stated that expectation of upcoming distractors increases distraction meaning distraction should be greater in the predictable low frequency condition.

Distraction did not differ between the predictable low-frequency condition and the unpredictable low-frequency condition, lending support to the second prediction that proactive control implementation is not driven solely by expectation of distractors. Distraction was also found to be significantly lower in the unpredictable high-frequency condition than in the predictable low-frequency condition supporting the suggestion that participants were not enacting proactive control in the predictable low-frequency condition. The results therefore converge with studies that find that control is not improved when upcoming conflict is expected (Augst et al., 2014; Bugg et al., 2015; Fernandez-Duque \& Knight, 2008; Kleinsorge, 2007; Moher \& Egeth, 2012; Schmidts et al., 2020). However, in contrast with Experiment 1 and previous emotional distraction studies (Kleinsorge, 2007; Schmidts et al., 2020) distraction was numerically but not statistically greater in the predictable low-frequency condition than in the unpredictable low-frequency condition. Thus, while expectation was not found to benefit control it was also not found to increase distraction in Experiment 2.

The present findings are in contrast with studies (typically assessing response conflict) which indicate that expectation improves control (Braver et al., 2003; Bugg \& Smallwood, 2016; Correa et al., 2008; Dreisbach et al., 2002; Havlíček et al., 2019; Liu \& Yeung, 2020; Logan \& Zbrodoff, 1982; Monsell et al., 2003). There are previous studies using response conflict tasks which have found evidence in favour of experience-based control (e.g. Bugg et al. 2015). However, the fact that evidence for expectation-based control stems mostly from response conflict tasks may suggest that different mechanisms may underlie the detection and resolution of response conflict and perceptual conflict (as induced in the present experiment) (Egner, 2014). In which case, the present findings may apply only to cognitive control in cases of competition between task-relevant and task-irrelevant emotional visual stimuli.

In addition to investigating the effect of expectation, Experiment 2 included a subjective measure of expectation, the post-experiment questionnaire. A substantial number of participants were unable to correctly report the distractor frequency, or whether distractors were ordered within their conditions. This is surprising, given that this information had been presented multiple times, during instructions and practice trials. Participants in both the predictable low-frequency and unpredictable high-frequency conditions were also less able to correctly indicate whether distractors were ordered or random, relative to the unpredictable 
low-frequency condition. Moreover, it appears that those in the unpredictable high-frequency condition were the most likely to incorrectly report distractor frequency. Hence participants in the unpredictable high-frequency condition, where control was most effectively applied, showed the least awareness of the predictability manipulation while those in the unpredictable low-frequency condition, where control was less effective ${ }^{12}$, indicated better awareness.

A greater proportion of participants in the predictable low-frequency condition also indicated that they were able to predict upcoming distractors and were more likely to report using a strategy to deal with intact-distractor trials. However, those in the predictable lowfrequency condition who indicated that they could predict distractors were no more able to ignore them than those who did not. Furthermore, when assessing the strategies reported by participants in the predictable low-frequency condition very few indicated that they prepared proactively for an upcoming intact-distractor trial. This could mean that strategic use of expectation is uncommon, and that awareness of the predictability manipulation did not improve performance. In all, the post-experiment questionnaire responses provide support for the arguments of Blais et al. (2012), who suggest that the explicit and implicit experiences of participants are distinct, and that participants' subjective feelings of expectation do not correlate with cognitive control.

The exploratory analyses completed in Experiment 2 also provide evidence which supplements the main conclusion that expectation of distractors does not improve control and may allude to the mechanism by which experience can modify control. Conflict adaptation (upregulation of control following exposure to conflict) could explain differences in distraction between the groups as there is greater opportunity for conflict adaptation to occur when distractors are frequent as opposed to infrequent (i.e. a greater likelihood of sequential intactdistractor trials). Hence, participants may be enacting control on a trial-by-trial basis, based on experiences on the previous trial, regardless of expectations. However, no interaction between Previous trial type and Distractor type was observed, suggesting that previous trial conflict did not lead to an upregulation of control in such a way as to specifically mitigate distraction. Additionally, in the predictable low-frequency condition, RTs did not indicate short term improvement and subsequent reduction in control after an intact-distractor trial. Hence no support was found for conflict adaptation as a mechanism through which experience biases control when distractors occur frequently. Exploratory analyses also indicated that distraction does not significantly decrease as a function of exposure to distractors over trial-blocks nor

12 Relative to the unpredictable high-frequency condition. 
within the first block of the experiment, as was expected in the unpredictable high-frequency condition if some amount of experience is necessary to modify control. Thus, there is no indication that a gradual change in control had occurred over time.

In sum, it is clear from both Experiments 1 and 2, that expectation of upcoming distractors did not lead to improved cognitive control of attention. In Experiment 2, however, distraction was not increased by expectation. The present series of experiments suggests that experience of past conflict rather than expectation of upcoming conflict biases cognitive control mechanisms to a more proactive style when distractors occur frequently.

\section{General Discussion}

Emotional distraction detracts from our ability to effectively navigate the visual world. To achieve our day-to-day goals, we implement cognitive control mechanisms to avoid emotional distraction. How effectively we control our attention is, however, determined by the current context, driving us to implement either proactive or reactive control mechanisms (Braver, 2012). Previous evidence indicates that distraction can be effectively avoided when emotional distractors occur frequently, presumably allowing us to enact proactive control mechanisms (Grimshaw et al., 2018). What is unclear, is whether the shift towards more effective proactive mechanisms is based on the expectation of future distraction, or because we

have gained experience in dealing with these distractors (Bugg et al., 2015; Bugg \& Crump, 2012; Schmidts et al., 2020).

Two experiments were conducted to assess whether expectation or experience biases control implementation towards being more proactive. Experiment 1 set out to assess whether participants could enact proactive control mechanisms when emotional and neutral distractors were infrequent, but expected, in the emotional irrelevant-distractor task. Participants were asked to identify whether the letter $\mathrm{K}$ or $\mathrm{N}$ was present in a letter array while negative or neutral task-irrelevant distractors were presented in the periphery. Distractors occurred on $25 \%$ of trials but occurred either in sequence (the predictable low-frequency condition in which expectation of upcoming distractors was high) or randomly (the unpredictable condition in which expectation of distractors was low). Results contradicted predictions as greater predictability of distractor occurrence did not lead to less distraction. In fact, analyses indicated that participants were more distracted when distractors were predictable, suggesting a paradoxical expectation effect in which distraction was increased rather than mitigated.

Experiment 2 set out to replicate Experiment 1. To maximise the likelihood of emotional distraction and the predictability of distractor occurrence, the emotional irrelevantdistractor task was modified to present distractors centrally, as opposed to presenting them in 
peripheral quadrants. An unpredictable high-frequency condition (distractors occurring on a random $75 \%$ of trials) was also added to allow me to directly assess the effect of distractor frequency (Grimshaw et al., 2018; Schmidts et al., 2020), providing a baseline against which to compare the effect of expectation. Distraction by both neutral and negative distractors was numerically, but not significantly, greater when distractors were predictable, compared to when they were equally frequent ( $25 \%$ of trials) but occurred randomly. Moreover, distraction in the predictable low-frequency condition was greater than that of the unpredictable high-frequency condition (with intermediate levels of distraction in the unpredictable low-frequency condition), indicating that experience rather than expectation of distractors, drove improved performance when distractor frequency was high. In comparing the predictable low-frequency condition to a typical frequency manipulation ${ }^{13}$, findings from Experiment 2 further indicated that task performance in the predictable low-frequency condition likely reflected the use of reactive, not proactive, control.

\section{Expectation and Experience}

Top-down cognitive control ${ }^{14}$ is thought to bias the competition between visual stimuli for attentional resources in an effortful and deliberate manner. According to the DMC framework, we implement effortful proactive control mechanisms when we have built an expectation of upcoming perceptual conflict and are therefore able to strategically and preemptively prepare for this conflict. The current results, however, suggest that we are not biased towards a more proactive style of control based on this expectation. Instead of expectation, the current findings suggest that it is the experience of previous conflict that modifies control. Since proactive top-down goal directed control is predicated on the assumption that it is strategic and deliberate (Braver, 2012); how are the current findings of experience-based control representative of deliberate top-down cognitive control of attention?

One possibility is that top-down control is not always voluntary and/or strategic and can arise implicitly through experience (Blais, 2010; Gaspelin \& Luck, 2018). From this perspective previous experience can, in a top-down manner, control attention to better achieve goals, and avoid distraction, when distractors occur frequently. The way in which experience is gained and is able to affect control automatically has been suggested to be the result of implicit statistical learning ${ }^{15}$ (Blais et al., 2012). Critically, this form of learning occurs quickly,

\footnotetext{
13 The comparison between the unpredictable low-frequency and unpredictable high-frequency conditions.

14 The term top-down control should be considered synonymous with cognitive control as illustrated by the DMC framework.

${ }^{15}$ Both statistical learning and implicit learning reflect automatic learning and are considered to be very similar processes (Perruchet \& Pacton, 2006). Note however that some may prefer the term statistical learning over
} 
automatically and without awareness or instruction (Fiser \& Aslin, 2001). In the visual modality, implicit statistical learning involves the learning of visual regularities, i.e. the repeated presence or absence of stimuli and the regularity with which they are arranged in space and time (Fiser \& Aslin, 2001; Zhao et al., 2013). The location and regularity of distractor occurrence are therefore prime candidates for statistical learning processes, allowing participants to gain experience of distractors to benefit future cognitive control.

Implicit changes in top-down control are also compatible with the dynamic nature of cognitive control, a key facet of the DMC framework. When contexts shift (as they constantly do) the relative costs and benefits of control mechanisms shift as well. However, it is unlikely that we respond to these shifts with frequent, deliberate and taxing strategic shifts in top-down control (Blais, 2010). Implicit modifications of control (based on experience of the current context) then provide a way for control to be implemented in a contextually appropriate and cost-effective way, without constant, deliberate shifts.

From an implicit learning standpoint, awareness of the statistical regularities present in the environment would be minimal. If we were aware of them, control would no longer be implicit. Hence, experience-driven control and implicit learning processes should be reflected in limited awareness. Conversely, if control is expectation-based and strategic, control effectiveness should be reflected in awareness and strategy in control implementation (Blais, 2010; Blais et al., 2012).

Blais et al. (2012) have previously shown that participants' ability to control their attention does not align with their awareness of the statistical regularities of conflict. The results of the Experiment 2 post-experiment questionnaire suggest a similar disconnect between participants' reported awareness of the predictability and distractor frequency manipulations, and their ability to avoid distraction. When asked to report whether they had implemented a strategy to control their attention, most participants (regardless of condition) indicated that they were not engaging in a strategy to prepare for upcoming distractors. Furthermore, questionnaire responses indicated that the majority of participants in the predictable low-frequency condition were aware that distractors were presented in a set order, but also that those who indicated that they could anticipate upcoming distractors were just as distracted as those who did not ${ }^{16}$. The fact that distraction was still high in the predictable low-frequency condition indicates that

implicit learning. For the present discussion, what is important is that both allow the individual to learn statistical regularities without awareness.

${ }^{16}$ In fact, they were numerically, but not statistically, more distracted. 
participants did not make use of the predictable order of distractions to implement control, regardless of whether they were aware of this manipulation or not.

In the unpredictable high-frequency condition, participants were the least accurate in identifying distractor frequency, with many also reporting that distractors were not presented on random trials. Here, where participants' control over attention was greatest, awareness of the frequency manipulation was the lowest. In contrast, in the unpredictable low-frequency condition (in which control was worse than in the unpredictable high-frequency condition), participants were more accurate in indicating the percentage of trials and whether they were ordered. Combined, these post-experiment questionnaire responses therefore indicate that the ability to avoid distraction was not influenced by the awareness of distractor frequency or predictability, but simply the frequency itself, which is indicative of implicit learning of distractor regularity rather than strategic use of expectations.

Participants in the present experiments therefore did not implement effortful proactive control when distractors were expected. Nor did they indicate that they were strategically implementing control or that they were aware of the environmental regularities (e.g. distractor frequency and/or distractor predictability) which implicate the need for effective control. This was true even when participants did implement effective (likely proactive) control mechanisms and could avoid distraction. Thus, to answer the question, "how are the current findings of experience-based control representative of deliberate top-down cognitive control of attention?" the present findings suggest that when distractors occur frequently, experience may implicitly bias individuals towards using proactive top-down control mechanisms based on implicit statistical learning.

\section{Evidence Against a Solely Experience-Based Account of Cognitive Control}

To be clear, I am not suggesting that proactive control is never driven by expectation (Blais, 2010). Indeed, the current experiments cannot provide conclusive evidence that either experience or expectation of distractors alone are sufficient drivers of proactive control. Were proactive control based solely on expectation, there would be less distraction when distractors were predictable and infrequent as compared to when distractors were unpredictable and infrequent. This pattern was not observed in either Experiment 1 or 2. Conversely, were proactive control based purely on previous experience, our ability to enact effective control would be reliant on the exposure to conflict in the environment. However, exploratory analyses of change in control over time indicated that, across all conditions, RTs during the first block did not decrease as participants progressed through the block, nor did distraction differ between 
the first and second trial blocks of either valence ${ }^{17}$. Moreover, even though the ability to control attention in the predictable condition was likely determined by the limited experience in the context of low distractor frequency, the paradoxical expectation effect observed in Experiment $1^{18}$ itself suggests that expectation still modifies control by increasing distraction. In the present investigation control was therefore likely dependent to some extent on the influence of both expectation and experience.

The current investigation is not alone in suggesting mixed use of expectation-based and experience-based control. Bugg et al. (2015), using the Stroop task, found that the Stroop effect was greater when conflict was infrequent but cued as opposed to when it was infrequent but uncued. However, cues did not alter the size of the Stroop effect when conflict was frequent and cued compared to when it was not cued. Expectation, therefore, still had some effect in the low conflict frequency condition but did not alter control when conflict was frequent. The overall difference in the magnitude of the Stroop effect (between the high and low conflict conditions) did however reflect that control was based on conflict frequency (i.e. experience). Moreover, when participants were provided with adequate incentive, performance on the first trial of a block was observed to be modified by a pre-block conflict likelihood cue while the remainder of trials indicated an experience-driven implementation of control (based on the frequency of conflict). Hence, participants made use of frequency information (experience) but also used pre-block cues (expectation) when incentivised to do so.

Similarly, the amount of time participants have available to prepare for upcoming distraction (e.g. the length of the cue-to-stimulus interval) has been previously shown to alter whether cues benefit control. Bugg and Smallwood (2016) observe a cue benefit in their first experiment only at the longest cue-to-stimulus interval (2000 ms). Logan \& Zbrodoff (1982) indicated that, using the cued above/below modified Stroop paradigm, that the benefit of not just pre-trial cues but the implementation of planned strategic control is dependent on the task environment (e.g. cue-to-stimulus delays) but also on the abilities of the individual completing the task (e.g. how practiced they had become with the task). What these studies show is that there can be cases where expectation-based control is simply not likely to occur based on the constraints of the context at the time (i.e. the characteristics of the experiment task). In all, the

\footnotetext{
${ }^{17}$ Perhaps the fact that training blocks presented distractors at the same frequency as the experiment proper, meant that participants did modify control based on experience prior to the first block of trials. In future studies, analysis or removal of the training trials may then be necessary to capture the experience-based performance change not seen in Experiment 2.

${ }^{18}$ The same difference is also seen in Experiment 2 but did not reach statistical significance $(p=.551)$.
} 
current and previous findings indicate that we may be able to use both experience and expectation to bias cognitive control towards or away from proactive mechanisms.

This implies that control can be based on expectation and/or experience dependent on the context. One could speculate that, since using effortful proactive control is still beholden to differences in costs and benefits of its implementation, effortful and strategic use of expectation-based control will be more likely when the benefit of doing so is increased. We may then make use of implicit experience-based mechanisms but also implement expectationbased control in an effortful and strategic manner if benefited enough to counteract the costs of doing so. As an example, motivation to perform well on experimental tasks, typically induced through rewarding good performance, may lead to more effective, possibly expectation-based, cognitive control, even when experience alone would otherwise bias participants towards reactive control (Liu \& Yeung, 2020; Walsh et al., 2018, 2019). From this perspective, the current results suggest that, in manipulations of distractor frequency, experience predominantly biases control, but given the appropriate contextual factors (e.g. motivation, adequate preparation time) an effect of expectation of distractors on control could possibly be observed.

\section{Sustained and Dynamic Proactive Control}

It is difficult to suggest specifically how experience modifies control since the influence of distraction frequency on cognitive control is, in many ways, still unclear. In the case of high distractor frequency for example, it is unclear whether experience-based proactive control occurs via sustained or more dynamic proactive control implementation. Sustained proactive control involves the constant goal monitoring and updating, leading to a "state-like" shift towards more effective control mechanisms (Braver, 2012). Conversely, dynamic proactive control involves transient pre-emptive control implementation. In experimental contexts, dynamic control such as this occurs just prior to the start of a trial, allowing participants to rapidly implement control in a phasic manner (Braver, 2012; Murphy et al., 2018).

In a recent electro-encephalography (EEG) study using the emotional irrelevantdistractor paradigm, Murphy et al. (2018) provides evidence which supports sustained proactive control. Distractors were presented (in quadrants) on either $25 \%$ or $75 \%$ of trials in the emotional irrelevant-distractor task. A typical effect of distractor frequency was observed in which distraction was greater in the $25 \%$ (low frequency) condition. Posterior-occipital Alpha $^{19}$, thought to be an inverse measure of attentional engagement (Boudewyn \& Carter,

${ }^{19}$ Oscillatory activity at $\sim 8$ to $12 \mathrm{~Hz}$, measured at occipital electrodes. 
2018), was also measured to assess the dynamic changes in control with EEG methods, allowing good temporal resolution of this change. Alpha power was tonically suppressed (overall lower alpha power) in the high relative to the low-frequency condition. Authors interpreted this result to indicate that proactive control was enacted in the high but not the lowfrequency group. Therefore, EEG evidence suggests that participants were able to engage in sustained attentional engagement (i.e. proactive control) when distractors occurred frequently as opposed to infrequently.

Conversely, it is harder to make the case for the use of dynamic proactive control mechanisms in response to high distractor frequency. In a typical distractor frequency manipulation, it is likely difficult to implement dynamic proactive control before the onset of the task and distractors, particularly if these distractors are uncued. Indeed Murphy et al. (2018) found that phasic changes in alpha power (dynamic changes in the period prior to distractor onset) did not differ between high and low frequency conditions, suggesting that dynamic proactive control was unaffected by distractor frequency.

That said, a possible method by which control may be altered dynamically is by conflict adaptation (Wiemers \& Redick, 2018). Conflict adaptation is the resulting upregulation of cognitive control in response to conflict detection leading to a conflict control loop in which control is upregulated based on the occurrence conflict (Krug \& Carter, 2012). On a trial-bytrial basis, conflict adaptation has been shown to lead to reductions in conflict effects (e.g. the Stroop effect) when the previous trials had been incongruent as opposed to congruent (Larson et al., 2009). A plausible explanation of increased control with increased conflict frequency is that there are more opportunities for conflict adaptation in high relative to low conflict frequency contexts simply due to the difference in number of subsequently occurring conflictpresent trials. (Blais, 2010). In cases of high rather than low conflict frequency, conflict adaptation effects should then lead to an overall improvement in control across trials by inducing more frequent implementations of dynamic proactive control.

Conversely, Experiment 2 exploratory analyses assessing whether conflict on previous trials influenced distraction showed that there was no improvement of control when intactdistractor trials were preceded by an intact-distractor trial in either of the unpredictable conditions of Experiment 2, nor was there improved task performance following an intactdistractor trial in the predictable low-frequency condition. Hence, conflict adaptation, as a mechanism of dynamic proactive control, is likely not occurring in the present experiments. Note, however, that Experiment 2 was not designed to investigate whether conflict adaptation 
mitigates distraction, and that the exploratory conflict adaptation analyses were underpowered as few trials were available.

The current study is not the first to show a frequency effect without evidence for conflict adaptation (Augst et al., 2014; Egner, 2007; Kunde \& Mauer, 2008; Schmidt et al., 2015; Torres-Quesada et al., 2013). Perhaps then, experience-based control occurs via different (possibly sustained) mechanisms than conflict adaptation possibly reflecting learning processes such as contingency learning that bypass, or occur in place of, conflict adaptation (Chiu \& Egner, 2019; Schmidt, 2013).

Future studies addressing whether conflict adaptation does in fact drive frequency effects, may indicate whether the greater cognitive control seen in cases of high distractor frequency is in some part due to dynamic processes of proactive control. A possible future experiment could investigate whether participants do adapt to the conflict on distractor present trials by manipulating the likelihood of sequential distractor-present trials. Using the present paradigm (with low distractor frequency), trials could be ordered in such a way as to create sequential distractor-present trial sequences (e.g. always presenting distractors in sequential trial pairs) creating a condition wherein conflict adaptation is induced on the second trial of a pair. Such a condition could then be compared to a condition similar to the predictable conditions of the present experiments where sequential distractor present trials never occur. Conflict adaptation based on a preceding trial would therefore only be possible in one condition. If control is greater in the condition in which conflict adaptation is possible, it would provide an indication that conflict adaptation could be acting as a method of dynamic proactive control.

\section{Selection History as an Alternative Explanation}

Previous studies which find evidence in favour of expectation-based proactive control (Aarts \& Roelofs, 2010; Gonthier et al., 2016; Logan \& Zbrodoff, 1982), have primarily implemented response conflict tasks (e.g. flanker and Stroop-like tasks). Conversely, emotional distraction studies which induce perceptual conflict, including the present experiments, have not found evidence of expectation-based proactive control (Kleinsorge, 2007; Schmidts et al., 2020). One could suggest that this difference in findings is driven by the fact that these studies induce conflict differently (i.e. response conflict or perceptual conflict). The present discussion should then address a possible alternative explanation for the present findings that may be unique to the use of irrelevant-distractor paradigms.

A plausible way in which distractor frequency can bias the allocation of attention, specific to the resolution of perceptual conflict, is selection history; the biasing of attentional 
allocation based on previous attentional selections (Theeuwes, 2019). Specifically, previous attentional selection is thought to modify priority maps, which dictate where attention is directed (Awh et al., 2012). This account suggests that statistical regularities, i.e. frequent distractors in the same location, can bias priority maps and allow for selective spatial suppression of irrelevant stimuli. As an example, Wang and Theeuwes (2018) found that when distractors occurred more frequently in some areas as opposed to others, participants were able to better ignore a future distractor presented in those areas. Hence, based only on the consistent occurrence of task-irrelevant stimuli in space, selection of task relevant stimuli could be greater at higher distractor frequencies.

This is, however, not reflective of experience-based top-down control. A solely selection history-based explanation of the current findings does not implicate a change in topdown control settings. Theeuwes (2019) in fact suggests that previous attentional selection implicitly biases the allocation of attention as a third factor, distinct to traditional bottom-up and top-down influences. Hence the current and similar previous findings (e.g. Kleinsorge, 2007; Schmidts et al., 2020) could be evidence of the effect of selection history (improved distractor suppression at high distractor frequency) rather than experience or expectation based proactive top-down control.

Theeuwes's view of selection history has received criticism, with some arguing that the effect of selection history does not necessarily exclude changes in top-down control implementation (Awh et al., 2012; Gaspelin \& Luck, 2018). Many models of spatial attention have also made efforts to incorporate top-down implicit control and selection history, specifically focusing on priority maps and spatial attention (Awh et al., 2012; Todd \& Manaligod, 2018; Wolfe et al., 2003). Selection history may then also act to modify top-down control settings in some way to bias the allocation of attentional resources in some goal directed fashion, possibly even as a mechanism of experience modified top-down control in irrelevantdistractor paradigms.

Future research could clarify the effect of selection history on the control of attention. Using the same design as Experiment 1, distraction by neutral and emotional task-irrelevant images could be compared between a condition in which distractors are presented in fixed locations and a condition in which they are not. For example, if distractors could occur at any location around a central letter array and not at any one location with regularity, participants would not be able to suppress distractor location accurately in comparison to a condition where distractors could only occur in a single or a few locations (e.g. presented above or below the letter array equally often). Equal distraction across conditions, would then be evidence against 
the action of selection history in modifying control. However, if distraction is substantially reduced when distractors occur regularly at a location relative to when their location is jittered, then participants may be suppressing distractor location based on a priority map and previous attentional selection.

\section{Are Emotional Stimuli Special?}

In addition to the findings regarding experience driven control, the present experiments indicate that emotional stimuli were more distracting than non-emotional stimuli, supporting previous studies which show that emotional stimuli can preferentially capture attentional resources even when these stimuli are irrelevant and presented alongside task-relevant stimuli (Carretié, 2014; Carretié et al., 2004; Grimshaw et al., 2020; Grimshaw et al., 2018; Pessoa, 2009; Schmidts et al., 2020). Experiment 2 also provides support for previous studies which indicate that increased distractor frequency enhances cognitive control and reduces distraction (Augst et al., 2014; Grimshaw et al., 2018; Schmidts et al., 2020). That said, greater distraction was found for trials containing emotional distractors than those containing neutral distractors, even in the unpredictable high-frequency condition. Thus, emotional stimuli were still more distracting than neutral stimuli even when proactive control was likely being implemented to direct attention.

Studies using emotional stimuli in conflict inducing tasks have previously shown that participants respond to emotional stimuli differently to neutral stimuli. As an example, Kunde and Mauer (2008) compared task performance on an irrelevant-distractor task, based on the valence of the distractor presented on the previous trial (either negative, neutral, or positive). Participants were required to respond to the colour of a frame surrounding an emotional image. Neutral images on the previous trial did not influence task performance on trials containing either negative or neutral images. Task performance was however slowed on trials containing both negative and positive images when the previous trial contained a negative image. Only negative images caused slower responding when the previous trial contained a positive image. Simply put, negative emotional stimuli on a previous trial did not lead to a reduction in distraction by an emotional image (negative or positive) whereas neutral images on previous trials did. Negative stimuli in fact appeared to facilitate attention to emotional stimuli on upcoming trials, suggesting that the conflict caused by the presence of emotional (particularly negative) stimuli did not reduce the attention given to irrelevant stimuli in subsequent trials (counter to what a conflict adaptation perspective would predict). Similarly the valence specific paradoxical expectation effect (Kleinsorge, 2007; Schmidts et al., 2020) contradicts an expectation-based control perspective, as distraction is increased rather than mitigated when 
negative but not neutral trials are cued. The present findings themselves indicate that emotional stimuli are not ignored as well as neutral stimuli at high distractor frequency suggesting experience-based proactive control is less effective when applied to emotional stimuli.

These findings illustrate that emotional stimuli elicit different control responses as compared to neutral stimuli. A possible reason why, is that these emotional stimuli, while irrelevant in context of the experimental tasks, are still salient because they are relevant to the individual more broadly (i.e. survival goals) as signals of harm and reward. Preferentially paying attention to emotional stimuli is after all adaptive (Pessoa, 2009). Emotional distraction may therefore present a unique challenge for cognitive control due to the inherent relevance and salience of emotional distractors.

\section{The Paradoxical Effect of Expectation}

Similar to the findings of Kleinsorge (2007) and Schmidts et al. (2020), the present experiments also found indications of a paradoxical expectation effect when distractors were expected in both negative and neutral trial blocks ${ }^{20}$. However, contrary to these previous findings the paradoxical expectation effect was only numerically and not statistically greater for negative distractors relative to neutral distractors. Nonetheless, the present findings indicate that expectation may have a harmful effect on control.

A possible explanation of the paradoxical expectation effect is that it is indicative of the attentional white bear effect; the allocation of attention to expected stimuli when these stimuli are to be ignored (Tsal \& Makovski, 2006). The white bear effect suggests that all stimuli incur automatic processing regardless of task relevance (Lahav et al., 2012; Moher \& Egeth, 2012; Tsal \& Makovski, 2006). Hence, if distractors are automatically processed, avoidance of distraction would require that they would subsequently need to be suppressed for participants to focus on the letter task (Geng, 2014) ${ }^{21}$. When a distractor is expected, the content of the distractor may be primed, leading to an increased engagement with the distractor (e.g. allocation of limited attentional resources) prior to suppression, slowing RT to the letter task.

Emotional stimuli may add a further complication as attention to these stimuli are prioritised. The valence specific nature paradoxical expectation effect in previous studies may then be indicative of the fact that there is value in being able to predict where and when emotional stimuli occur to allow them to be attended to adequately. Further investigation into

\footnotetext{
${ }^{20}$ Statistically significant in the Experiment 1 Distraction-index analysis and numerically so in Experiment 2.

${ }^{21}$ This suggests a predominantly reactive mechanism of control, however, there are accounts (Geng, 2014) which indicate that suppression in this way can be prepared for proactively.
} 
emotion-specific paradoxical expectation effects could further our understanding of the processes of selective attention and emotional distraction. A future study could compare distraction by neutral and emotional images when cues were and were not valid for upcoming trial valence (e.g. by including uninformative and incorrect pre-trial cues in the emotional irrelevant-distractor task). If distraction is increased by the fact that cues increase the attentional engagement with upcoming distractors, then distraction should be greater when distractors are cued and not when distractors are falsely cued or uncued. The valence-specific nature of the paradoxical expectation effect can be addressed in the comparison between emotional and neutral distractors which follow valid and invalid cues. If slowing is valence specific, the difference in distraction between invalidly and validly cued distractors should be greater for emotional than for neutral distractors.

\section{Caveats and Limitations}

Findings of the current experiments suggest that expectation alone does not bias individuals to implement cognitive control. However, the caveats and limitations which accompany the present findings should also be acknowledged. Caveats include the use of sequential distractor presentation as a method of inducing expectation and the fact that the current results cannot speak to the effect of expectation on the avoidance of positive distractors. Limitations include the lack of a predictable high-frequency condition and the possibility that participants had been cued to the proportion of distractors in all conditions during the experiment instructions.

\section{Manipulating Predictability}

A caveat to the current findings is that the way in which the present experiments made distractors predictable (as inspired by the alternate run procedure) is novel in an emotional distraction study. This method was chosen to avoid possible priming effects and the fact that cues have been shown to provide differing levels of benefit, depending on motivation and cueto-stimulus interval. Additionally, using the alternate run inspired manipulation meant that little modification of the emotional irrelevant-distractor paradigm was required, allowing the unpredictable conditions to essentially be replications of previous distractor frequency manipulations. However, it may be that the sequential presentation of trials in the predictable low-frequency condition did not induce expectation of an upcoming distractor as effectively as pre-trial cueing (Koch, 2003). Indeed, while cueing of distractors has sometimes been ineffective in the past, particularly when using emotional stimuli as task-irrelevant distractors, there are still studies which show a cue benefit. There is then the possibility that that 
expectation-based proactive control occurs when pre-trial cues are used but not when expectation is built via trial sequence.

A simple way to address whether the current predictability manipulation induces expectation differently to explicit cues is to replicate Experiment 2 whilst including pre-trial cues in all conditions. Distractors would then occur rarely and randomly in all conditions, but, would be prefaced by either valid (a predictable condition) or uninformative (an unpredictable condition) cues. If cues are found to reduce distraction relative to the unpredictable condition (as expected from an expectation-based account of control), then the predictability manipulation in the present experiments may not be inducing expectation as intended. If not, then the replication would provide further support to the conclusion of the present experiments that expectation alone does not drive proactive control implementation.

\section{Positive Emotional Images}

The current experiments also do not speak to the effect of increased expectation and experience in the case of high arousal positive stimuli. There are previous studies which find no difference between positive and negative images in terms of control implementation when distractors are frequent (Grimshaw et al., 2018; Grimshaw et al., 2020). However, these stimuli are still indicative of different environmental signals (i.e. reward and threat) and may also differ in how they direct attention (Kleinsorge, 2007; Kunde \& Mauer, 2008). Replication of the current experiments using high arousal erotic stimuli (as used in Grimshaw et al., 2018) could clarify whether expectation of upcoming conflict differentially affects control between threatening and rewarding stimuli. Additionally, such a future study could also indicate whether a paradoxical expectation effect still exists when positive emotional stimuli are used instead of negative emotional stimuli. Kleinsorge (2009) indicated that this is not the case as cued negative but not positive task-irrelevant images worsened participants ability to indicate whether a mathematical equation had been correctly solved. A paradoxical expectation effect may then be linked to expectation of upcoming threat rather than only the fact that we are motivated to attend to any emotional images with sufficient ratings of arousal ${ }^{22}$.

\section{A Predictable High-Frequency Condition}

A limitation of the design of Experiment 2 is the lack of a predictable high-frequency condition. This condition could have shown whether increased predictability would have improved control implementation over and above the effect of frequency and therefore whether there are simultaneous and independent effects of experience and expectation. Yet, a high-

22 Taken here to as the measure of arousal taken by the Self-assessment manakin scale described in the methods. 
experience, high-expectation condition may not show any additional improvement when distractors are frequent enough to elicit proactive control, as shown by Bugg et al. (2015). The present study did not include such a condition since the question of interest concerned the effect of expectation in a context where distractors occurred infrequently (i.e. when distraction was more likely than not). The unpredictable high-frequency condition which was added in Experiment 2 acted as a proactive control "baseline" against which to compare the effects of expectation. A predictable high-frequency condition was therefore orthogonal to the goals of the second experiment but may have indicated that expectation does influence control if proactive control is already likely. An extension of the current study could be to replicate the second experiment in a balanced $2 \times 2$ design, to allow for the assessment of the independent contributions of experience and expectation to control.

\section{Unintended Foreknowledge of Distractor Frequency}

Another limitation is participant foreknowledge of distractor frequency. Participants in all conditions of Experiment 2 were told the frequency with which distractors would occur during the experiment ${ }^{23}$. This was necessary to provide equal knowledge of the task across conditions, since those in the predictable low-frequency condition could easily realise the distractor frequency $(25 \%)$ when they were informed about the predictability manipulation (a distractor on every fourth trial). This inadvertently creates a design similar to that used by Bugg and colleagues (Bugg et al., 2015) wherein participants were explicitly provided with the proportion of congruent trials prior to a block. An exclusively expectation-based implementation of control may then still have occurred (as would be in line with the DMC framework), as participants could expect upcoming distractor frequency to be low in the predictable conditions and therefore choose to not implement proactive control since the benefit to task performance would not outweigh the cost. This may also explain the lack of experience induced change in distraction over time in the unpredictable high-frequency condition of Experiment 2, suggesting participants adopted sustained proactive control at the start of the experiment. If implicit learning is in fact taking place, foreknowledge of distractor frequency would not be necessary to see an effect of distractor frequency. Thus, replicating the current experiments without informing participants of frequency beforehand may provide a more valid assessment of experience-based control.

\section{Future Directions}

\footnotetext{
${ }^{23} \mathrm{~A}$ similar situation existed in Experiment 1 but distractor frequency was only known in the predictable condition. Participants in the predictable condition may therefore not have enacted proactive control since they knew distraction was infrequent prior to the start of the experiment (i.e. were still influenced by expectations).
} 
Modifications of the present paradigm as mentioned above can likely address methodological limitations and caveats. However, the current findings also pose new avenues for future investigation. Present and previous experiments indicate that expectation and experience are likely both used in context to bias control to be more proactive. This raises the question; under what circumstances are we likely to implement control based on expectation alongside, or in place of, experience?

The DMC framework assumes that individuals are motivated to perform optimally. However previous studies have indicated that participants' control over attention is greater when they are rewarded for good task performance (Chiew \& Braver, 2016; Padmala \& Pessoa, 2011; Walsh et al., 2018, 2019) possibly suggesting that participants are not inherently motivated to perform optimally. The link between control and reward can be reflected in the cost/benefit analysis of the DMC framework. When considered this way, the benefit gained from proactive control use may simply be insufficient to prompt participants away from reactive control mechanisms if previous experience indicates that reactive control will suffice. We may very well see that participants would implement proactive control based on their expectations, even when distractor frequency is low, if they are adequately motivated by benefits of optimal performance.

Using the same emotional irrelevant-distractor paradigm as used in Experiment 2, a future study could present infrequent and frequent distractors alongside some method of building expectation of upcoming distraction (e.g. cues or the sequential predictability manipulation). The findings of the present experiment would suggest that experience would drive participants to implement reactive and proactive control dependent on distractor frequency and cues would have no effect. Participants could then be provided with monetary reward for good performance (high and low-frequency incentivised conditions) or not receive a performance contingent reward (high and low-frequency not-incentivized conditions).

Expectation of upcoming distractors may bias then participants to implement expectation-based proactive control, even if distractors are infrequent, given that they have adequate incentive to implement more costly control mechanisms (i.e. when the benefits outweigh the costs). Cue benefits (e.g. lower distraction) could then be predicted in the incentivised conditions but not in the not-incentivised conditions. When distractors occur frequently, and proactive control is already likely. Lesser distraction based on motivation to perform well would then indicate that improvements in cognitive control based on experience and expectation are additive. A simple set of control conditions would be a frequency manipulation (high and low) wherein distractors are uncued but performance is incentivised, 
allowing for the comparison between cued, incentivised task performance and uncued incentivised task performance at either distraction frequency ${ }^{24}$.

\section{Conclusion}

In this thesis I assessed whether expectation of upcoming distraction can bias cognitive control mechanisms to a more proactive style. In my first experiment, I compared distraction by neutral and emotional stimuli when participants were and were not able to expect infrequent distractors. In the second experiment, I set out to replicate the results of the first while holding distractor location constant and including a condition in which distractors were frequent and unpredictable, to test the effects of distractor frequency.

I found that expectation clearly does not lead to lesser distraction by either neutral or emotional images. In Experiment 1 expectation of upcoming distractors in fact worsened distraction by both neutral and emotional images. Hence, present findings suggest that when distractors are frequent, it is not the expectation of upcoming distraction alone that allows us to avoid distractors more effectively. Rather, findings suggest that distractor frequency can influence control through the experience we gain with distractors. However, both expectation and experience are likely able to modify the type of control mechanisms we enact when faced with attentional conflict. Cognitive control is therefore suggested to be driven by many interacting factors, dependent on the context in which conflict occurs. The valence of visual stimuli itself may be one such factor, posing a unique challenge in terms of how we control our attention.

\footnotetext{
${ }^{24}$ A post-experiment questionnaire could also assess whether motivation led to a more strategic use of the cues (expectation) if the Experiment 2 methods are expressly replicated.
} 


\section{References}

Aarts, E., \& Roelofs, A. (2010). Attentional control in anterior cingulate cortex based on probabilistic cueing. Journal of Cognitive Neuroscience, 23(3), 716-727. https://doi.org/10.1162/jocn.2010.21435

Andreadis, N., \& Quinlan, P. T. (2010). Task switching under predictable and unpredictable circumstances. Attention, Perception \& Psychophysics, 72(7), 1776-1790. https://doi.org/10.3758/APP.72.7.1776

Aron, A. R. (2011). From reactive to proactive and selective control: Developing a richer model for stopping inappropriate responses. Biological Psychiatry, 69(12), e55-68. https://doi.org/10.1016/j.biopsych.2010.07.024

Augst, S., Kleinsorge, T., \& Kunde, W. (2014). Can we shield ourselves from task disturbance by emotion-laden stimulation? Cognitive, Affective, \& Behavioral Neuroscience, 14(3), 1009-1025. https://doi.org/10.3758/s13415-013-0243-x

Awh, E., Belopolsky, A. V., \& Theeuwes, J. (2012). Top-down versus bottom-up attentional control: A failed theoretical dichotomy. Trends in Cognitive Sciences, 16(8), 437443. https://doi.org/10.1016/j.tics.2012.06.010

Blais, C. (2010). Implicit versus deliberate control and its implications for awareness. In Effortless attention: A new perspective in the cognitive science of attention and action (pp. 141-157). MIT Press.

Blais, C., Harris, M. B., Guerrero, J. V., \& Bunge, S. A. (2012). Rethinking the role of automaticity in cognitive control. Quarterly Journal of Experimental Psychology, 65(2), 268-276. https://doi.org/10.1080/17470211003775234

Botvinick, M. M., Braver, T. S., Barch, D. M., Carter, C. S., \& Cohen, J. D. (2001). Conflict monitoring and cognitive control. Psychological Review, 108(3), 624-652. https://doi.org/10.1037/0033-295x.108.3.624

Boudewyn, M. A., \& Carter, C. S. (2018). Electrophysiological correlates of adaptive control and attentional engagement in patients with first episode schizophrenia and healthy young adults. Psychophysiology, 55(3), e12820. https://doi.org/10.1111/psyp.12820

Braver, T. S., Paxton, J. L., Locke, H. S., \& Barch, D. M. (2009). Flexible neural mechanisms of cognitive control within human prefrontal cortex. Proceedings of the National Academy of Sciences, 106(18), 7351-7356. https://doi.org/10.1073/pnas.0808187106 
Braver, T. S., Reynolds, J. R., \& Donaldson, D. I. (2003). Neural mechanisms of transient and sustained cognitive control during task switching. Neuron, 39(4), 713-726. https://doi.org/10.1016/S0896-6273(03)00466-5

Braver, T. S. (2012). The variable nature of cognitive control: A dual-mechanisms framework. Trends in Cognitive Sciences, 16(2), 106-113. https://doi.org/10.1016/j.tics.2011.12.010

Braver, T. S., Gray, J. R., \& Burgess, G. C. (2007). Explaining the many varieties of working memory variation: Dual mechanisms of cognitive control. In Variation in working memory (pp. 76-106). Oxford University Press.

Brosch, T., Pourtois, G., \& Sander, D. (2010). The perception and categorisation of emotional stimuli: A review. Cognition and Emotion, 24(3), 377-400. https://doi.org/10.1080/02699930902975754

Bugg, J. M., Diede, N. T., Cohen-Shikora, E. R., \& Selmeczy, D. (2015). Expectations and experience: Dissociable bases for cognitive control? Journal of Experimental Psychology: Learning, Memory, and Cognition, 41(5), 1349-1373. https://doi.org/10.1037/xlm0000106

Bugg, J. M., Jacoby, L. L., \& Toth, J. P. (2008). Multiple levels of control in the Stroop task. Memory \& Cognition, 36(8), 1484-1494. https://doi.org/10.3758/MC.36.8.1484

Bugg, J. M., \& Smallwood, A. (2016). The next trial will be conflicting! Effects of explicit congruency pre-cues on cognitive control. Psychological Research, 80(1), 16-33. https://doi.org/10.1007/s00426-014-0638-5

Bugg, J. M., \& Crump, M. J. C. (2012). In support of a distinction between voluntary and stimulus-driven control: a review of the literature on proportion congruent effects. Frontiers in Psychology, 3. https://doi.org/10.3389/fpsyg.2012.00367

Carretié, L. (2014). Exogenous (automatic) attention to emotional stimuli: A review. Cognitive, Affective, \& Behavioral Neuroscience, 14(4), 1228-1258. https://doi.org/10.3758/s13415-014-0270-2

Carretié, L., Hinojosa, J. A., Martín-Loeches, M., Mercado, F., \& Tapia, M. (2004). Automatic attention to emotional stimuli: Neural correlates. Human Brain Mapping, 22(4), 290-299. https://doi.org/10.1002/hbm.20037

Chiew, K. S., \& Braver, T. S. (2016). Reward favours the prepared: Incentive and taskinformative cues interact to enhance attentional control. Journal of Experimental Psychology. Human Perception and Performance, 42(1), 52-66. https://doi.org/10.1037/xhp0000129 
Chiu, Y.C., \& Egner, T. (2019). Cortical and subcortical contributions to context-control learning. Neuroscience \& Biobehavioral Reviews, 99, 33-41. https://doi.org/10.1016/j.neubiorev.2019.01.019

Connor, C. E., Egeth, H. E., \& Yantis, S. (2004). Visual attention: bottom-up versus topdown. Current Biology, 14(19), R850-R852. https://doi.org/10.1016/j.cub.2004.09.041

Correa, Á., Rao, A., \& Nobre, A. C. (2008). Anticipating conflict facilitates controlled stimulus-response selection. Journal of Cognitive Neuroscience, 21(8), 1461-1472. https://doi.org/10.1162/jocn.2009.21136

De Pisapia, N., \& Braver, T. S. (2006). A model of dual control mechanisms through anterior cingulate and prefrontal cortex interactions. Neurocomputing, 69(10), 1322-1326. https://doi.org/10.1016/j.neucom.2005.12.100

Desimone, R., \& Duncan, J. (1995). Neural mechanisms of selective visual attention. Annual Review of Neuroscience, 18(1), 193-222. https://doi.org/10.1146/annurev.ne.18.030195.001205

Dreisbach, G., Haider, H., \& Kluwe, R. H. (2002). Preparatory processes in the taskswitching paradigm: evidence from the use of probability cues. Journal of Experimental Psychology: Learning, Memory, and Cognition, 28(3), 468. https://doi.org/10.1037//0278-7393.28.3.468

Duthoo, W., De Baene, W., Wühr, P., \& Notebaert, W. (2012). When predictions take control: the effect of task predictions on task switching performance. Frontiers in Psychology, 3. https://doi.org/10.3389/fpsyg.2012.00282

Egner, T. (2007). Congruency sequence effects and cognitive control. Cognitive, Affective, \& Behavioral Neuroscience, 7(4), 380-390. https://doi.org/10.3758/CABN.7.4.380

Egner, T. (2014). Creatures of habit (and control): A multi-level learning perspective on the modulation of congruency effects. Frontiers in Psychology, 5. https://doi.org/10.3389/fpsyg.2014.01247

Egner, T., \& Hirsch, J. (2005). Cognitive control mechanisms resolve conflict through cortical amplification of task-relevant information. Nature Neuroscience, 8(12), 1784-1790. https://doi.org/10.1038/nn1594

Fernandez-Duque, D., \& Knight, M. (2008). Cognitive control: Dynamic, sustained, and voluntary influences. Journal of Experimental Psychology. Human Perception and Performance, 34(2), 340-355. https://doi.org/10.1037/0096-1523.34.2.340 
Fiser, J., \& Aslin, R. N. (2001). Unsupervised statistical learning of higher-order spatial structures from visual scenes. Psychological Science, 12(6), 499-504. https://doi.org/10.1111/1467-9280.00392

Forster, S., \& Lavie, N. (2008). Attentional capture by entirely irrelevant distractors. Visual Cognition, 16(2-3), 200-214. https://doi.org/10.1080/13506280701465049

Gaspelin, N., \& Luck, S. J. (2018). “Top-down” does not mean "voluntary.” Journal of Cognition, 1(1). https://doi.org/10.5334/joc.28

Geng, J. J. (2014). Attentional mechanisms of distractor suppression. Current Directions in Psychological Science, 23(2), 147-153. https://doi.org/10.1177/0963721414525780

Geyer, T., Müller, H. J., \& Krummenacher, J. (2008). Expectancies modulate attentional capture by salient color singletons. Vision Research, 48(11), 1315-1326. https://doi.org/10.1016/j.visres.2008.02.006

Goldfarb, L., \& Henik, A. (2013). The effect of a preceding cue on the conflict solving mechanism. Experimental Psychology, 60(5), 347-353. http://dx.doi.org/10.1027/1618-3169/a000205

Gonthier, C., Braver, T. S., \& Bugg, J. M. (2016). Dissociating proactive and reactive control in the Stroop task. Memory \& Cognition, 44(5), 778-788. https://doi.org/10.3758/s13421-016-0591-1

Gratton, G., Coles, M. G. H., \& Donchin, E. (1992). Optimizing the use of information: Strategic control of activation of responses. Journal of Experimental Psychology: General, 121(4), 480-506. http://dx.doi.org.helicon.vuw.ac.nz/10.1037/00963445.121.4.480

Gratton, G., Cooper, P., Fabiani, M., Carter, C. S., \& Karayanidis, F. (2018). Dynamics of cognitive control: Theoretical bases, paradigms, and a view for the future. Psychophysiology, 55(3), e13016. https://doi.org/10.1111/psyp.13016

Grimshaw, G. M., Devue, C., Kranz, L. S., Jenkins, D. C., O’Connel, A., \& Carmel, D. (2020). Cognitive control of emotional distractors in central vision [Manuscript submitted for publication]. School of Psychology, Victoria University of Wellington.

Grimshaw, G. M., Kranz, L. S., Carmel, D., Moody, R. E., \& Devue, C. (2018). Contrasting reactive and proactive control of emotional distraction. Emotion, 18(1), 26-38. https://doi.org/10.1037/emo0000337

Gupta, R., Hur, Y.-J., \& Lavie, N. (2016). Distracted by pleasure: Effects of positive versus negative valence on emotional capture under load. Emotion, 16(3), 328-337. https://doi.org/10.1037/emo0000112 
Havlíček, O., Müller, H. J., \& Wykowska, A. (2019). Distract yourself: Prediction of salient distractors by own actions and external cues. Psychological Research, 83(1), 159174. https://doi.org/10.1007/s00426-018-1129-x

Hutchison, K. A., Bugg, J. M., Lim, Y. B., \& Olsen, M. R. (2016). Congruency precues moderate item-specific proportion congruency effects. Attention, Perception, \& Psychophysics, 78(4), 1087-1103. https://doi.org/10.3758/s13414-016-1066-y

Jiang, J., Heller, K., \& Egner, T. (2014). Bayesian modeling of flexible cognitive control. Neuroscience and Biobehavioral Reviews, 46 Pt 1, 30-43. https://doi.org/10.1016/j.neubiorev.2014.06.001

Jiménez, L., Abrahamse, E., Méndez, C., \& Braem, S. (2019). Does incidental sequence learning allow us to better manage upcoming conflicting events? Psychological Research. https://doi.org/10.1007/s00426-019-01201-6

Kalanthroff, E., Henik, A., Derakshan, N., \& Usher, M. (2016). Anxiety, emotional distraction, and attentional control in the Stroop task. Emotion, 16(3), 293-300. https://doi.org/10.1037/emo0000129

Katsuki, F., \& Constantinidis, C. (2014). Bottom-up and top-down attention: different processes and overlapping neural systems. The Neuroscientist, 20(5), 509-521. https://doi.org/10.1177/1073858413514136

Kleinsorge, T. (2007). Anticipatory modulation of interference induced by unpleasant pictures. Cognition and Emotion, 21(2), 404-421. https://doi.org/10.1080/02699930600625032

Kleinsorge, T. (2009). Anticipation selectively enhances interference exerted by pictures of negative valence. Experimental Psychology, 56(4), 228-235. http://dx.doi.org.helicon.vuw.ac.nz/10.1027/1618-3169.56.4.228

Koch, I. (2003). The role of external cues for endogenous advance reconfiguration in task switching. Psychonomic Bulletin \& Review, 10(2), 488-492. https://doi.org/10.3758/BF03196511

Kray, J., \& Lindenberger, U. (2000). Adult age differences in task switching. Psychology and Aging, 15(1), 126-147. https://doi.org/10.1037/0882-7974.15.1.126

Krug, M. K., \& Carter, C. S. (2012). Conflict control loop theory of cognitive control. The Neuroscience of Attention: Attentional Control and Selection. https://doi.org/10.1093/acprof:oso/9780195334364.003.0011 
Kunde, W., \& Mauer, N. (2008). Sequential modulations of valence processing in the emotional stroop task. Experimental Psychology, 55(3), 151-156. https://doi.org/10.1027/1618-3169.55.3.151

Lahav, A., Makovski, T., \& Tsal, Y. (2012). White bear everywhere: Exploring the boundaries of the attentional white bear phenomenon. Attention, Perception, \& Psychophysics, 74(4), 661-673. https://doi.org/10.3758/s13414-012-0275-2

Lakens, D. (2013). Calculating and reporting effect sizes to facilitate cumulative science: A practical primer for t-tests and ANOVAs. Frontiers in Psychology, 4. https://doi.org/10.3389/fpsyg.2013.00863

Lang, P. J., Bradley, M. M., \& Cuthbert, B. N. (2008). International affective picture system (IAPS): Affective ratings of pictures and instruction manual. University of Florida, Gainesville. FL, Technical Report A-8.

Larson, M. J., Kaufman, D. A. S., \& Perlstein, W. M. (2009). Neural time course of conflict adaptation effects on the Stroop task. Neuropsychologia, 47(3), 663-670. https://doi.org/10.1016/j.neuropsychologia.2008.11.013

LeDoux, J. (2012). Rethinking the emotional brain. Neuron, 73(4), 653-676. https://doi.org/10.1016/j.neuron.2012.02.004

Liu, C., \& Yeung, N. (2020). Dissociating expectancy-based and experience-based control in task switching. Journal of Experimental Psychology: Human Perception and Performance, 46(2), 131-154. https://doi.org/10.1037/xhp0000704

Logan, G. D., \& Zbrodoff, N. J. (1979). When it helps to be misled: Facilitative effects of increasing the frequency of conflicting stimuli in a Stroop-like task. Memory \& Cognition, 7(3), 166-174. https://doi.org/10.3758/BF03197535

Logan, G. D., \& Zbrodoff, N. J. (1982). Constraints on strategy construction in a speeded discrimination task. Journal of Experimental Psychology: Human Perception and Performance, 8(4), 502. https://doi.org/10.1037/0096-1523.8.4.502

Marini, F., Berg, B. van den, \& Woldorff, M. G. (2015). Reward prospect interacts with trialby-trial preparation for potential distraction. Visual Cognition, 23(1-2), 313-335. https://doi.org/10.1080/13506285.2015.1023387

Melara, R. D., \& Algom, D. (2003). Driven by information: A tectonic theory of Stroop effects. Psychological Review, 110(3), 422-471. https://doi.org/10.1037/0033295X.110.3.422

Mitchell, D. G. V., Richell, R. A., Leonard, A., \& Blair, R. J. R. (2006). Emotion at the expense of cognition: Psychopathic individuals outperform controls on an operant 
response task. Journal of Abnormal Psychology, 115(3), 559-566.

https://doi.org/10.1037/0021-843X.115.3.559

Moher, J., \& Egeth, H. E. (2012). The ignoring paradox: Cueing distractor features leads first to selection, then to inhibition of to-be-ignored items. Attention, Perception, \& Psychophysics, 74(8), 1590-1605. https://doi.org/10.3758/s13414-012-0358-0

Monsell, S., Sumner, P., \& Waters, H. (2003). Task-set reconfiguration with predictable and unpredictable task switches. Memory \& Cognition, 31(3), 327-342. https://doi.org/10.3758/BF03194391

Most, S. B., Smith, S. D., Cooter, A. B., Levy, B. N., \& Zald, D. H. (2007). The naked truth: Positive, arousing distractors impair rapid target perception. Cognition and Emotion, 21(5), 964-981. https://doi.org/10.1080/02699930600959340

Müller, H. J., Geyer, T., Zehetleitner, M., \& Krummenacher, J. (2009). Attentional capture by salient color singleton distractors is modulated by top-down dimensional set. Journal of Experimental Psychology: Human Perception and Performance, 35(1), 1-16. https://doi.org/10.1037/0096-1523.35.1.1

Murphy, J., Devue, C., Corballis, P. M., \& Grimshaw, G. M. (2018). Proactive control of emotional distraction: Evidence from alpha suppression. Open Science Framework. https://doi.org/10.31219/osf.io/vuk6j

Ohman, A., Flykt, A., \& Esteves, F. (2001). Emotion drives attention: detecting the snake in the grass. Journal of experimental psychology: general, 130(3), 466. https://doi.org/10.1037/0096-3445.130.3.466

Padmala, S., \& Pessoa, L. (2011). Reward reduces conflict by enhancing attentional control and biasing visual cortical processing. Journal of Cognitive Neuroscience, 23(11), 3419-3432.https://doi.org/10.1162/jocn_a_00011

Peirce, J., Gray, J. R., Simpson, S., MacAskill, M., Höchenberger, R., Sogo, H., Kastman, E., \& Lindeløv, J. K. (2019). PsychoPy2: Experiments in behavior made easy. Behavior Research Methods, 51(1), 195-203. https://doi.org/10.3758/s13428-018-01193-y

Perruchet, P., \& Pacton, S. (2006). Implicit learning and statistical learning: One phenomenon, two approaches. Trends in Cognitive Sciences, 10(5), 233-238. https://doi.org/10.1016/j.tics.2006.03.006

Pessoa, L. (2005). To what extent are emotional visual stimuli processed without attention and awareness? Current Opinion in Neurobiology, 15(2), 188-196. https://doi.org/10.1016/j.conb.2005.03.002 
Pessoa, L. (2009). How do emotion and motivation direct executive control? Trends in Cognitive Sciences, 13(4), 160-166. https://doi.org/10.1016/j.tics.2009.01.006

Pool, E., Brosch, T., Delplanque, S., \& Sander, D. (2016). Attentional bias for positive emotional stimuli: A meta-analytic investigation. Psychological Bulletin, 142(1), 79106. https://doi.org/10.1037/bu10000026

Pourtois, G., Schettino, A., \& Vuilleumier, P. (2013). Brain mechanisms for emotional influences on perception and attention: What is magic and what is not. Biological Psychology, 92(3), 492-512. https://doi.org/10.1016/j.biopsycho.2012.02.007

Purmann, S., \& Pollmann, S. (2015). Adaptation to recent conflict in the classical color-word Stroop-task mainly involves facilitation of processing of task-relevant information. Frontiers in Human Neuroscience, 9. https://doi.org/10.3389/fnhum.2015.00088

Rogers, R., \& Monsell, S. (1995). Costs of a predictable switch between simple cognitive tasks. Journal of Experimental Psychology: General, 124, 207-231. https://doi.org/10.1037/0096-3445.124.2.207

Schmidt, J. R. (2013). Questioning conflict adaptation: Proportion congruent and Gratton effects reconsidered. Psychonomic Bulletin \& Review, 20(4), 615-630. https://doi.org/10.3758/s13423-012-0373-0

Schmidt, J. R., Notebaert, W., \& Bussche, E. V. D. (2015). Is conflict adaptation an illusion? Frontiers in Psychology, 6. https://doi.org/10.3389/fpsyg.2015.00172

Schmidts, C., Foerster, A., Kleinsorge, T., \& Kunde, W. (2020). Proactive control of affective distraction: Experience-based but not expectancy-based. Cognition, 194, 104072. https://doi.org/10.1016/j.cognition.2019.104072

Schupp, H. T., Stockburger, J., Codispoti, M., Junghofer, M., Weike, A. I., \& Hamm, A. O. (2007). Selective visual attention to emotion. Journal of Neuroscience, 27(5), 10821089. https://doi.org/10.1523/JNEUROSCI.3223-06.2007

Stroop, J. R. (1935). Studies of interference in serial verbal reactions. Journal of Experimental Psychology, 18(6), 643-662. https://doi.org/10.1037/h0054651

Theeuwes, J. (2013). Feature-based attention: It is all bottom-up priming. Philosophical Transactions of the Royal Society B: Biological Sciences, 368(1628), 20130055. https://doi.org/10.1098/rstb.2013.0055

Theeuwes, J. (2019). Goal-driven, stimulus-driven, and history-driven selection. Current Opinion in Psychology, 29, 97-101. https://doi.org/10.1016/j.copsyc.2018.12.024 
Todd, R. M., \& Manaligod, M. G. M. (2018). Implicit guidance of attention: The priority state space framework. Cortex, 102, 121-138. https://doi.org/10.1016/j.cortex.2017.08.001

Torres-Quesada, M., Funes, M. J., \& Lupiáñez, J. (2013). Dissociating proportion congruent and conflict adaptation effects in a Simon-Stroop procedure. Acta Psychologica, 142(2), 203-210. https://doi.org/10.1016/j.actpsy.2012.11.015

Tsal, Y., \& Makovski, T. (2006). The attentional white bear phenomenon: The mandatory allocation of attention to expected distractor locations. Journal of Experimental Psychology: Human Perception and Performance, 32(2), 351-363. https://doi.org/10.1037/0096-1523.32.2.351

Ullsperger, M., Bylsma, L. M., \& Botvinick, M. M. (2005). The conflict adaptation effect: It's not just priming. Cognitive, Affective, \& Behavioral Neuroscience, 5(4), 467-472. https://doi.org/10.3758/CABN.5.4.467

Walsh, A. T., Carmel, D., \& Grimshaw, G. M. (2019). Reward elicits cognitive control over emotional distraction: Evidence from pupillometry. Cognitive, Affective, \& Behavioral Neuroscience, 19(3), 537-554. https://doi.org/10.3758/s13415-01800669-w

Walsh, A. T., Carmel, D., Harper, D., \& Grimshaw, G. M. (2018). Motivation enhances control of positive and negative emotional distractions. Psychonomic Bulletin \& Review, 25(4), 1556-1562. https://doi.org/10.3758/s13423-017-1414-5

Wang, B., \& Theeuwes, J. (2018). How to inhibit a distractor location? Statistical learning versus active, top-down suppression. Attention, Perception, \& Psychophysics, 80(4), 860-870. https://doi.org/10.3758/s13414-018-1493-z

Wang, L., LaBar, K. S., Smoski, M., Rosenthal, M. Z., Dolcos, F., Lynch, T. R., Krishnan, R. R., \& McCarthy, G. (2008). Prefrontal mechanisms for executive control over emotional distraction are altered in major depression. Psychiatry Research: Neuroimaging, 163(2), 143-155. https://doi.org/10.1016/j.pscychresns.2007.10.004

Wiemers, E. A., \& Redick, T. S. (2018). Working memory capacity and intra-individual variability of proactive control. Acta Psychologica, 182, 21-31. https://doi.org/10.1016/j.actpsy.2017.11.002

Willenbockel, V., Sadr, J., Fiset, D., Horne, G. O., Gosselin, F., \& Tanaka, J. W. (2010). Controlling low-level image properties: The SHINE toolbox. Behavior Research Methods, 42(3), 671-684. https://doi.org/10.3758/BRM.42.3.671 
Wolfe, J. M., Butcher, S. J., Lee, C., \& Hyle, M. (2003). Changing your mind: On the contributions of top-down and bottom-up guidance in visual search for feature singletons. Journal of Experimental Psychology: Human Perception and Performance, 29(2), 483-502. https://doi.org/10.1037/0096-1523.29.2.483

Zhao, J., Al-Aidroos, N., \& Turk-Browne, N. B. (2013). Attention is spontaneously biased toward regularities. Psychological Science, 24(5), 667-677. https://doi.org/10.1177/0956797612460407 


\section{Appendix A}

\section{IAPS Images and Ratings}

Table A1

Mean (SD) ratings for valence and arousal, as taken from the female ratings in (Lang et al., 2008) for the images used in experiments 1 and 2

\begin{tabular}{|c|c|c|}
\hline & Valence & Arousal \\
\hline Image Number & Mean (SD) & Mean (SD) \\
\hline \multicolumn{3}{|l|}{ Neutral } \\
\hline 2026 & $4.85(0.85)$ & $3.38(1.88)$ \\
\hline 2102 & $5.13(0.99)$ & $2.92(2.02)$ \\
\hline 2221 & $4.33(1.24)$ & $3.05(1.83)$ \\
\hline 2305 & $5.14(0.86)$ & $3.05(1.88)$ \\
\hline 2393 & $4.92(1.05)$ & $2.95(1.95)$ \\
\hline 2397 & $4.93(1.05)$ & $2.56(1.65)$ \\
\hline 2411 & $5.06(0.89)$ & $2.96(1.9)$ \\
\hline 2512 & 4.780 .94() & 3.29 (1.94) \\
\hline 2593 & $5.73(1.5)$ & $3.22(1.96)$ \\
\hline 2595 & $4.97(1.31)$ & $3.65(1.85)$ \\
\hline 2745.1 & $5.38(1.22)$ & $3.31(1.95)$ \\
\hline 2840 & $4.9(1.23)$ & $2.55(1.76)$ \\
\hline \multicolumn{3}{|l|}{ Negative } \\
\hline 3015 & $1.34(0.71)$ & $6.11(2.87)$ \\
\hline 3030 & $1.51(1.07)$ & $7.13(1.88)$ \\
\hline 3059 & $1.47(0.95)$ & $6.5(2.52)$ \\
\hline 3103 & $1.71(1.02)$ & $6.6(2.07)$ \\
\hline 3131 & $1.4(0.84)$ & $6.62(2.3)$ \\
\hline 3140 & $1.5(0.97)$ & $6.94(1.68)$ \\
\hline 3150 & $1.98(1.54)$ & $6.94(2.07)$ \\
\hline 3195 & $1.79(1.06)$ & $6.42(2.53)$ \\
\hline 3550.1 & $1.92(1.34)$ & $6.62(1.9)$ \\
\hline 9253 & $1.6(0.99)$ & $5.65(2.58)$ \\
\hline 9405 & 1.59 (1.02) & $6.77(2.22)$ \\
\hline 9420 & $1.87(1.54)$ & $6.1(2.37)$ \\
\hline
\end{tabular}




\section{Appendix B}

Experiment 1 Supplementary Distraction index Analysis.

To further clarify the RT results, distraction was assessed directly by comparing distraction indices (RT(distractor-present) - RT(distractor-absent)), which are presented alongside matching RTs in Table 1. Distraction indices were entered into a 2 (Valence: Neutral, Negative) $\times 2$ (Predictability: Predictable, Unpredictable) mixed ANOVA. A main effect of valence was observed, $F(1,102)=12.28, p<.001, \eta_{p}{ }^{2}=.12$, where negative distractors $(M=27, S D=43)$ elicited higher distraction indices than neutral distractors $(M=$ $12, S D=29)$. In support of the Distractor type $\times$ Predictability interaction in the RT analysis, a main effect of predictability was also observed, $F(1,102)=4.19, p=.043, \eta_{p}{ }^{2}=.04$, wherein, counter to hypotheses, distraction indices were lower in the unpredictable $(M=14$, $S D=33)$ than predictable condition $(M=25, S D=40)$. As in the RT analysis distraction is overall greater in the predictable condition in which participants were able to predict distractor occurrence with $100 \%$ certainty. Moreover, the Predictability $\times$ Valence interaction was non-significant, $F(1,102)=2.01, p=.159, \eta_{p}{ }^{2}=.02$, indicating that the effect of valence is not reduced by the expectation, as was predicted. 


\section{Appendix C}

\section{Experiment 2 Post-Experiment Questionnaire ${ }^{25}$}

"Note that all the information given to you during the instructions and practice trials was

$$
\text { truthful." }
$$

How often were intact images present?

$\%$ of trials in a block

Did you feel as if the images were presented to you in a set order? (Circle an option)

$$
\text { Yes No }
$$

Were you able to predict when an intact image would be present on the following trial? (Circle an option)

Yes No

Did you use any strategy to prepare for the intact images on upcoming trials? (Circle an option)
Yes
No

If yes; what was this strategy?

${ }^{25}$ Participants were reminded what is meant by an intact image and that the pre-experiment instructions were truthful. They were also provided with an explanation that "set order" referred to the order of scrambled and intact images within a block of trials. 


\section{Appendix D}

Participant Answers to Question 5 of the Experiment 2 Post-experiment Questionnaire.

\section{Predictable condition}

\section{Distractor Focussed}

$\underline{\text { Task Focussed }}$

$\underline{\text { Active Preparation }}$
- "Concentrate at not focussing on anything in particular"

- "Squinted or even closed my eyes every fourth go when the intact image was present"

- "Squinted my eyes a bit more to avoid having to look at the full intact image"

- "To avoid looking at the centre of the image in case it was a gory image, and focus on where the letter would appear"

- "Focussing on the white cross"

- "When I knew an intact image was coming, I would look at the letters more"

- "Emotionally bracing myself I guess"

- "I told myself there was going to be one, so I wasn't surprised"

\section{Unpredictable low-frequency condition}

$\underline{\text { Distractor Focussed }}$
- "Avoid looking at it so I could see the letter. Focus my attention on the top and bottom rows" 
- "I blurred my vision so wouldn't be as shocked by the gory intact images"

- "I didn't look directly on the screen, kinda "through" it so my side vision could see everything but not directly at it"

- "Ignore it, (well try)"

- "Blur the image in my head and only gaze above or below the photo"

- "Just focus on the middle"

- "I tried to focus on the centre of the image, so I could get a full visual range to see where and what the letter was"

$\underline{\text { Task Focussed }}$

None

Active Preparation

None

\section{Unpredictable high-frequency condition}

$\underline{\text { Distractor Focussed }}$

$\underline{\text { Task Focussed }}$

Active Preparation
- "I tried to block images, but I noticed the sound of the beep affected me more in making my choices"

- “Just focussed on the cross, didn't look at the images"

- "Checked the top row first, bottom row was covered by doing that"

None 


\title{
Appendix E
}

\author{
Experiment 2 Preregistration and Amendment
}

\section{Data collection}

Have any data been collected for this study already? Note: 'Yes' is a discouraged answer for this preregistration form.

No, no data have been collected for this study yet.

\section{Hypothesis}

Existing evidence shows that attention is biased towards emotionally charged stimuli, even when they are irrelevant to our current goals. Recent studies also show that, when distractors are frequent, distraction by emotional stimuli is decreased. An explanation for this increased attentional control, is that high distractor frequency signals increased need for control over and the enactment of more effective but taxing control mechanisms. However, studies also show that cueing distractors or making their occurrence predictable, does not alter distraction. We therefore hypothesize that foreknowledge of upcoming distractors and experience of distractor frequency may trigger the enactment of different control mechanisms. This study will directly compare the effects of predictability and increased distractor frequency on distraction by emotional images.

\section{Dependent variable}

Mean Reaction time (RT): Time taken to respond to a simple letter identification task in milliseconds, in each condition.

Distraction index (DI): The difference in mean reaction time between distractor present trials and distractor absent trials.

Accuracy: The number of incorrect answers to the letter task in each condition.

\section{Conditions}

How many and which conditions will participants be assigned to?

Participants will be assigned to one of three conditions:

1) A predictable low frequency condition

2) An unpredictable low frequency condition, and

3) An unpredictable high frequency condition

All participants will complete an emotional distractor task wherein they are tasked to identify a target letter in an array. The letter array will flank either an irrelevant intact image (neutral or negative) or a pixel scrambled image. The study will consist of 4 blocks of 48 trials ( 2 
blocks each of neutral and negative distractors). Block order will be counterbalanced. In the predictable condition, distractors will occur on every fourth trial. In the unpredictable conditions, distractors will occur randomly throughout a block of trials on either $25 \%$ (unpredictable low frequency condition) or on $75 \%$ of trials (unpredictable high frequency condition).

\section{Analyses}

RT: Mean RT values will be compared using a 3 (Condition: Low Frequency unpredictable, High Frequency Unpredictable, Predictable) $\times 2$ (Distractor Valence: Negative, Neutral) x 2 (Distractor type: Present, Absent) Mixed ANOVA. If there are significant interactions with distractor type, we will test the distractor present and distractor absent conditions separately, to determine if conditions have similar baseline levels of performance when distractors are absent.

DI: Distraction indexes will be compared using a 3 (Condition: Low Frequency Unpredictable, High Frequency Unpredictable, Predictable) $\times 2$ (Blocked Distractor Valence: Negative, Neutral) Mixed ANOVA. Main effects of Condition will be followed up the oneway ANOVA, with Tukey correction, to compare distraction across groups. Significant interactions will be followed up with paired t-tests to compare negative and neutral distraction in each condition, and one-way ANOVA (with Tukey correction) to compare groups on negative and neutral distraction separately.

Errors: Accuracy analysis will be used to test for the existence of a speed-accuracy trade-off. The number of errors will be compared using a 3 (Condition: Low Frequency Unpredictable, High Frequency Unpredictable, Predictable) $\times 2$ (Blocked Distractor Valence: Negative, Neutral) Mixed ANOVA. Accuracy is expected to be high.

\section{Outliers and Exclusions}

Trials wherein participant responses occur within 200ms of stimulus onset will be excluded as anticipatory responses.

Outliers will be defined as individuals having mean distraction of more than 3 standard deviations from the group mean in any one condition.

An accuracy criterion will be implemented whereby participants will be removed from analysis if accuracy on any block falls below $75 \%$.

\section{Sample Size}

$\mathrm{G}^{*}$ power analysis indicated 93 participants total would be needed to attain an effect size of Cohen's $\mathrm{f}=.33$ with power $=.9$. When adjusting this to adequately counterbalance the experiment, 96 participants (32 per condition) would need to be recruited. This sample size is 
large enough to detect main effects of both the within and between subject factors as well as interaction effects.

\section{Other}

Participants will be asked in a post-experiment questionnaire if they noticed any pattern to the appearance of distractors, to estimate how often they appeared, and to indicate whether they adopted any strategy using this information. This will be used to assess whether participants make use of the frequency and order information (of which they are made aware in the beginning of the experiment). Exploratory analyses will use participant awareness as a variable in the same analyses described above.

\section{Name}

Cognitive control over emotional distraction: The roles of predictability and experience

\section{Preregistration amendment}

This document is an amendment to the preregistration posted on August 2, 2019 for the project "Emotional distraction alpha power". At the time of writing this amendment, data collection has yet to be completed and no analysis of the existing data has been attempted. The purpose of this amendment is to correct an error made in the original submission and to clarify the exclusion criteria prior to data analysis.

\section{Error analysis}

The preregistration should have stated that the error data will be assessed using a 3 (Condition: Low Frequency Unpredictable, High Frequency Unpredictable, Predictable) $\times 2$ (Blocked Distractor Valence: Negative, Neutral) x 2 (Distractor type: Present, Absent) Mixed ANOVA.

\section{Exclusion criteria}

The incorrect exclusion criteria were used in the original preregistration. To ensure that the study remain in line with existing studies using the same/similar methods, the exclusion criteria will be modified to the following. Participants will be removed from the analysis if mean accuracy for neutral or negative blocks is found to be below 70\%. Additionally, participants will be removed if overall accuracy on the task falls below $75 \%$. Trials wherein participant responses occur within $200 \mathrm{~ms}$ of stimulus onset will be excluded as anticipatory responses and will not be included in the RT analysis.

Again, these changes are set in motion during data collection during a review of the existing study materials, prior to the analysis of any data. 